Supporting Information

\title{
Axially Chiral peri-Xanthenoxanthenes as a Circularly Polarized Luminophore
}

Kazuto Takaishi,* Sakiko Hinoide, Tomoki Matsumoto, and Tadashi Ema*

Division of Applied Chemistry, Graduate School of Natural Science and Technology, Okayama University, Tsushima, Okayama 700-8530, Japan

\section{Table of contents}

1. General information S2

2. Synthetic methods and characterization S3

$\begin{array}{ll}\text { 3. X-ray structures } & \text { S18 }\end{array}$

$\begin{array}{ll}\text { 4. DFT calculations } & \text { S19 }\end{array}$

$\begin{array}{ll}\text { 5. FL and CPL spectra } & \text { S22 }\end{array}$

$\begin{array}{lll}\text { 6. } & { }^{1} \mathrm{H} \text { NMR and }{ }^{13} \mathrm{C} \text { NMR spectra } & \text { S24 }\end{array}$

$\begin{array}{ll}\text { 7. Coordinates of optimized structures } & \text { S59 }\end{array}$

$\begin{array}{lll}\text { 8. } & \text { References } & \text { S78 }\end{array}$ 


\section{General information}

Melting points were measured on a Yanaco melting point apparatus (uncorrected). Optical rotations were measured on a Horiba SEPA-300. IR spectra were recorded on a Shimadzu IRAffinity-1. ${ }^{1} \mathrm{H}$ NMR and ${ }^{13} \mathrm{C}$ NMR spectra were recorded on a JEOL JNM-ECS400. Data are reported as follows: chemical shifts in ppm using the residual solvent peak as an internal standard, integration, multiplicity $(\mathrm{s}=$ singlet, $\mathrm{d}=$ doublet, $\mathrm{t}=$ triplet, $\mathrm{q}=$ quartet, quin $=$ quintet, sext $=$ sextet, $\mathrm{m}=$ multiplet $)$, and coupling constants $(\mathrm{Hz})$. High-resolution mass spectra were performed on a Agilent G6520+G4240. UV-Vis spectra were recorded on a Shimadzu UV-2600. CD spectra were recorded on a JASCO J-1500. Fluorescence spectra were recorded on a JASCO FP-750. Absolute fluorescence quantum yields were determined by a JASCO FP-6500. CPL spectra were measured on a JASCO CPL-200. TLC analyses were carried out on glass sheets coated with Merck Silica gel $60 \mathrm{~F}_{254}(0.25 \mathrm{~mm})$, and visualization was accomplished with UV light. Column chromatography was performed on silica gel (Fuji Silysia BW-127 ZH, 100-270 mesh). 


\section{Synthetic methods and characterization}

Scheme S1. Synthesis of Chiral peri-Xanthenoxanthenes 2 and 3

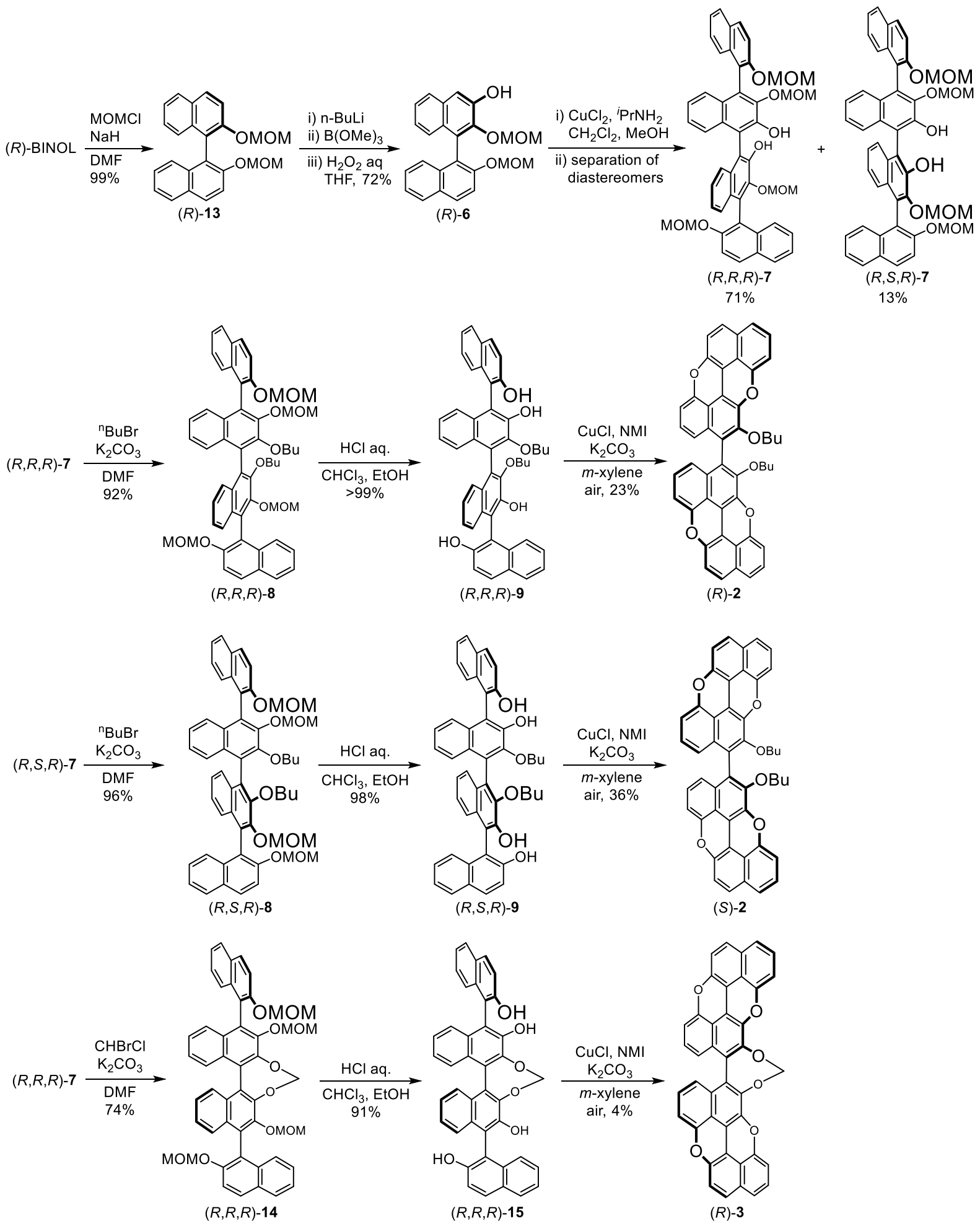


Scheme S2. Synthesis of Chiral peri-Xanthenoxanthenes 4 and 5

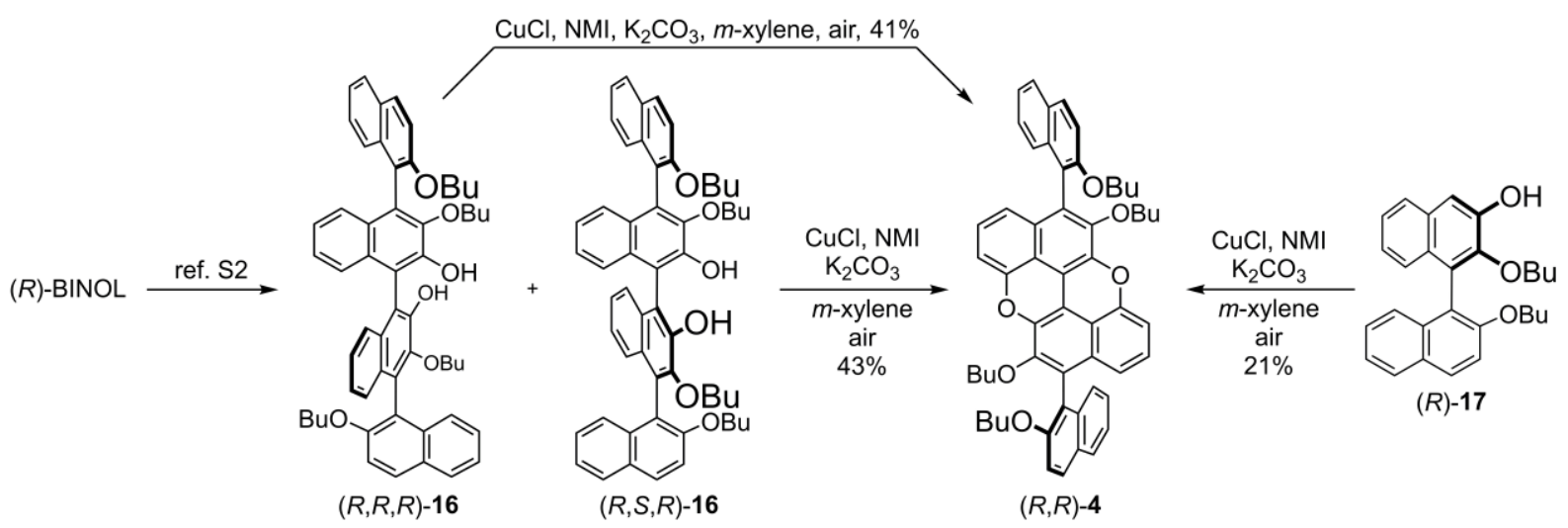

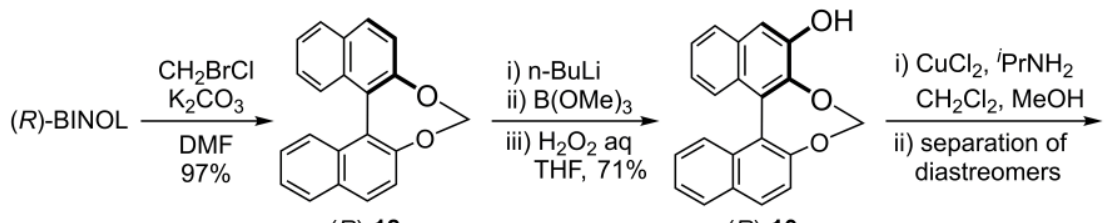

$(R)-18$

$(R)-10$
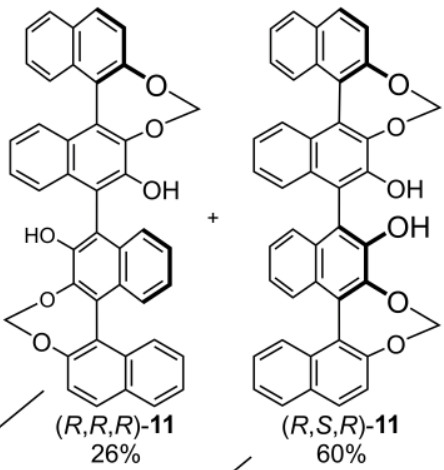
$\mathrm{CuCl}, \mathrm{NMI}, \mathrm{K}_{2} \mathrm{CO}_{3}$
$m$-xylene, air, $7 \%$

$\mathrm{CuCl}, \mathrm{NMI}, \mathrm{K}_{2} \mathrm{CO}_{3}$

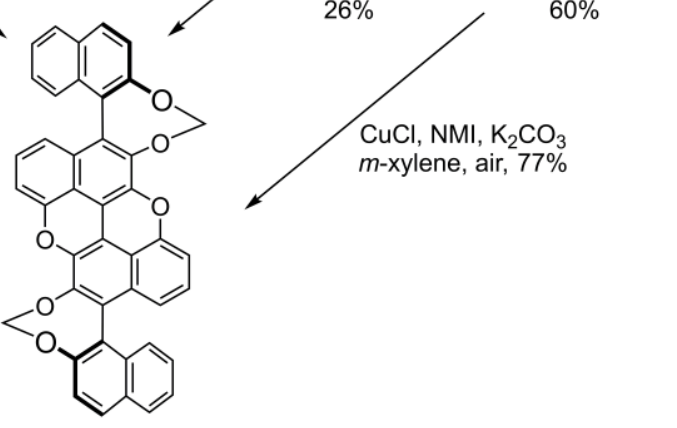

$(R, R)-5$

Compounds $(R)-\mathbf{1 3}^{\mathrm{S} 1},(R, R, R)-\mathbf{1 6}^{\mathrm{S} 2},(R, S, R)-16^{\mathrm{S} 2},(R)-\mathbf{1 7}^{\mathrm{S} 2}$, and $(R)-\mathbf{1 8}^{\mathrm{S} 3}$ were synthesized according to the literature. $(S)-\mathbf{3},(S)-\mathbf{4}$, and $(S, S)-\mathbf{5}$ were synthesized in a similar manner to those for $(R)-\mathbf{3},(R)-\mathbf{4}$, and $(R, R)-5$, respectively, with the exception that $(S)$-BINOL was used as a starting material. 


\section{Synthesis of $(R)-6$}

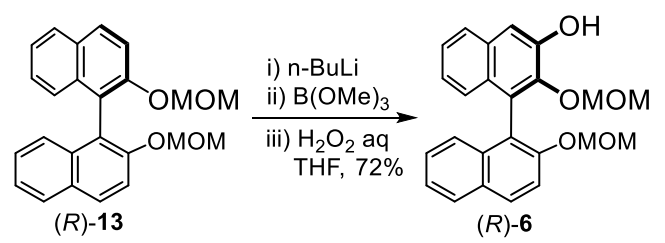

To a solution of $(R)-13(5.00 \mathrm{~g}, 13.4 \mathrm{mmol})$ in dry THF $(45 \mathrm{~mL}), 1.6 \mathrm{M} \mathrm{n}$-BuLi in hexane $(8.9 \mathrm{~mL}, 14$ mmol) was added dropwise at $-78{ }^{\circ} \mathrm{C}$ under $\mathrm{N}_{2}$ atmosphere. The solution was stirred at $0{ }^{\circ} \mathrm{C}$ for $1 \mathrm{~h}$. $\mathrm{B}(\mathrm{OMe})_{3}(2.98 \mathrm{~mL}, 25.8 \mathrm{mmol})$ was added to the solution at $-78^{\circ} \mathrm{C}$, and the reaction mixture was stirred at room temperature for $13 \mathrm{~h} . \mathrm{H}_{2} \mathrm{O}_{2}(30 \mathrm{wt} \%$ solution in water, $41 \mathrm{~mL})$ was added to the solution at $0{ }^{\circ} \mathrm{C}$, and the solution was stirred at $50{ }^{\circ} \mathrm{C}$ for $3 \mathrm{~h}$. Then saturated aqueous $\mathrm{Na}_{2} \mathrm{SO}_{3}$ was added. The organic solvent was evaporated in vacuo, and EtOAc was added. The organic layer was separated and washed successively with water and brine (twice). After dried over $\mathrm{Na}_{2} \mathrm{SO}_{4}$, the solvent was evaporated in vacuo to give a residue. The residue was purified by column chromatography $\left(\mathrm{SiO}_{2}, \mathrm{CHCl}_{3} / \mathrm{EtOAc}=10 / 1\right)$ to afford $(R)-6$ (3.77 $\mathrm{g}, 9.66 \mathrm{mmol}, 72 \%)$ as an orange viscous oil. The NMR data were in agreement with those reported previously. ${ }^{\mathrm{S} 4}$

\section{Synthesis of $(R, R, R)-7$ and $(R, S, R)-7$}

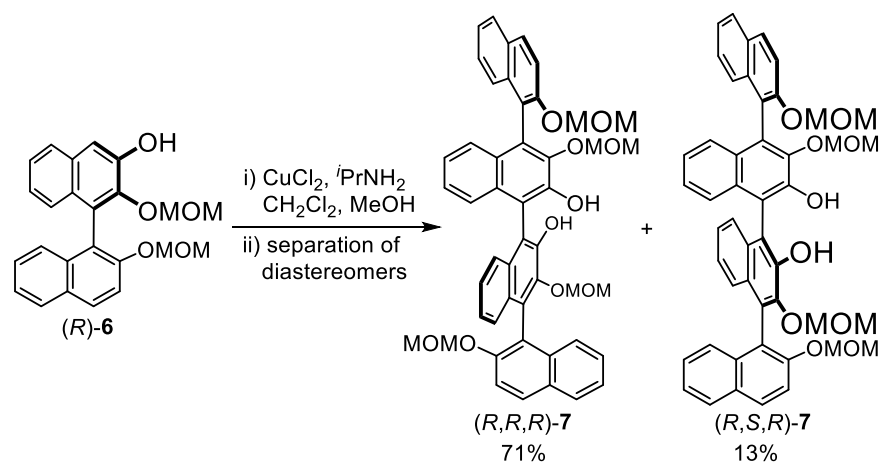

To a mixture of $\mathrm{CuCl}_{2}(561 \mathrm{mg}, 4.17 \mathrm{mmol})$ in dry $\mathrm{MeOH}(12 \mathrm{~mL}), i-\mathrm{PrNH}_{2}(0.50 \mathrm{~mL}, 5.8 \mathrm{mmol})$ was added under $\mathrm{N}_{2}$ atmosphere with ice-bath cooling. After an hour, a solution of (R)-6 (983 mg, $2.52 \mathrm{mmol}$ ) in dry $\mathrm{CH}_{2} \mathrm{Cl}_{2}(12 \mathrm{~mL})$ was added, and the reaction mixture was stirred at $0{ }^{\circ} \mathrm{C}$ for $19 \mathrm{~h}$. The reaction mixture was poured into a mixed solvent of $1 \mathrm{M} \mathrm{HCl}$ aq. and $\mathrm{CHCl}_{3}$. The aqueous layer was extracted with $\mathrm{CHCl}_{3}$ (twice). The organic layers were combined, washed with water (twice) and dried over $\mathrm{Na}_{2} \mathrm{SO}_{4}$ and evaporated to give a residue. The residue was purified by column chromatography $\left(\mathrm{SiO}_{2}, \mathrm{CHCl}_{3} / \mathrm{EtOAc}=\right.$ $5 / 1)$ to afford $(R, R, R)-7(700 \mathrm{mg}, 899 \mu \mathrm{mol}, 71 \%)$ as an orange powder and $(R, S, R)-7$ (129 mg, $166 \mu \mathrm{mol}$, $13 \%)$ as a yellow powder.

$(R, R, R)-7: \mathbf{m p} 100-103{ }^{\circ} \mathrm{C} ;[\alpha]_{\mathrm{D}}{ }^{21}=+108\left(\mathrm{CHCl}_{3}, c 0.10\right)$; IR (KBr) 3059, 2955, 2901, 2826, 1620, 1508, 1422, 1240, 1152, 1063, 1015, 920, 812, $752 \mathrm{~cm}^{-1} ;{ }^{\mathbf{1}} \mathbf{H} \mathbf{~ N M R}\left(400 \mathrm{MHz}, \mathrm{CDCl}_{3}\right) \delta 3.24(\mathrm{~s}, 6 \mathrm{H}), 3.27$ $(\mathrm{s}, 6 \mathrm{H}), 4.83(\mathrm{AB} \mathrm{q}, \Delta v=43.1 \mathrm{~Hz}, J=6.2 \mathrm{~Hz}, 4 \mathrm{H}), 5.19(\mathrm{AB} \mathrm{q}, \Delta v=27.5 \mathrm{~Hz}, J=6.7 \mathrm{~Hz}, 4 \mathrm{H}), 6.98-7.15$ $(\mathrm{m}, 4 \mathrm{H}), 7.20-7.28(\mathrm{~m}, 4 \mathrm{H}), 7.30-7.45(\mathrm{~m}, 8 \mathrm{H}), 7.63(\mathrm{~d}, J=9.0 \mathrm{~Hz}, 2 \mathrm{H}), 7.92(\mathrm{~d}, J=8.1 \mathrm{~Hz}, 2 \mathrm{H}), 8.02(\mathrm{~d}$, 
$J=9.0 \mathrm{~Hz}, 2 \mathrm{H}) ;{ }^{13} \mathrm{C}$ NMR $\left(100 \mathrm{MHz}, \mathrm{CDCl}_{3}\right) \delta 56.2,57.6,95.7,99.8,116.6,117.4,121.1,124.0,124.5$, 125.1, 125.8, 126.0, 126.3, 126.5, 126.9, 128.0, 128.9, 130.0, 130.1, 131.5, 134.1, 145.0, 146.5, 152.8; HR MS $\left(\mathrm{ESI}^{+}\right)$Calcd for $\mathrm{C}_{48} \mathrm{H}_{42} \mathrm{O}_{10} \mathrm{Na}: 801.2670[\mathrm{M}+\mathrm{Na}]^{+}$. Found: 801.2670.

$(R, S, R)-7: \mathbf{~ m p ~} 125-128^{\circ} \mathrm{C} ;[\alpha]_{\mathrm{D}^{21}}=+24\left(\mathrm{CHCl}_{3}, c\right.$ 0.10); IR (KBr) 3059, 2955, 2903, 2826, 1622, 1506, 1422, 1240, 1150, 1074, 1013, 920, 810, $758 \mathrm{~cm}^{-1}$; ${ }^{1} \mathbf{H}$ NMR (400 MHz, $\left.\mathrm{CDCl}_{3}\right) \delta 3.21(\mathrm{~s}, 6 \mathrm{H}), 3.23$ (s, $6 \mathrm{H}), 4.78(\mathrm{~d}, J=6.0 \mathrm{~Hz}, 2 \mathrm{H}), 4.93(\mathrm{~d}, J=6.1 \mathrm{~Hz}, 2 \mathrm{H}), 5.16(\mathrm{AB} \mathrm{q}, \Delta v=24.9 \mathrm{~Hz}, J=6.7 \mathrm{~Hz}, 4 \mathrm{H}), 6.96(\mathrm{~s}$, $2 \mathrm{H}), 7.14$ (ddd, $J=1.6,6.8,8.3 \mathrm{~Hz}, 2 \mathrm{H}), 7.24(\mathrm{~d}, J=8.2 \mathrm{~Hz}, 2 \mathrm{H}), 7.29(\mathrm{ddd}, J=1.6,6.8,8.3 \mathrm{~Hz}, 2 \mathrm{H}$ ), $7.36-7.46(\mathrm{~m}, 8 \mathrm{H}), 7.65(\mathrm{~d}, J=9.1 \mathrm{~Hz}, 2 \mathrm{H}), 7.93(\mathrm{dd}, J=2.0,6.8 \mathrm{~Hz}, 2 \mathrm{H}), 8.02(\mathrm{~d}, J=9.0 \mathrm{~Hz}, 2 \mathrm{H}) ;{ }^{13} \mathbf{C}$ NMR $\left(100 \mathrm{MHz}, \mathrm{CDCl}_{3}\right) \delta 56.2,57.5,95.5,99.6,116.3,117.1,120.7,124.1,124.4,125.1,125.7,126.0$, 126.4, 126.5, 127.0, 128.1, 128.9, 129.9, 130.1, 131.5, 134.1, 144.8, 146.6, 153.0; HR MS (ESI $\left.{ }^{+}\right)$Calcd for $\mathrm{C}_{48} \mathrm{H}_{42} \mathrm{O}_{10} \mathrm{Na}: 801.2670[\mathrm{M}+\mathrm{Na}]^{+}$. Found: 801.2642.

\section{Synthesis of $(R, R, R)-8$}
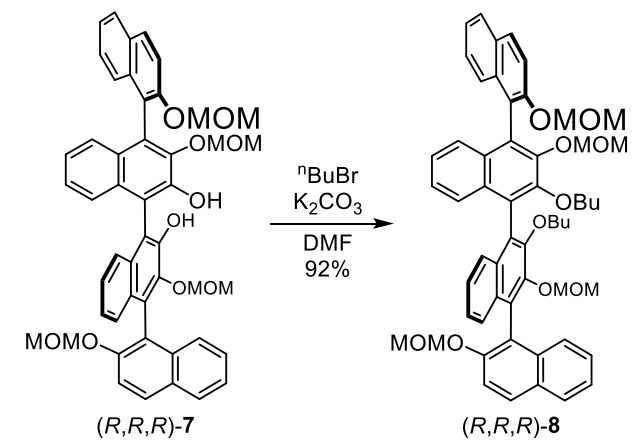

A mixture of $(R, R, R)-7(201 \mathrm{mg}, 258 \mu \mathrm{mol}), \mathrm{n}-\mathrm{BuBr}(0.11 \mathrm{~mL}, 1.0 \mathrm{mmol})$, and $\mathrm{K}_{2} \mathrm{CO}_{3}(167 \mathrm{mg}, 1.21$ mmol) in dry DMF $(0.5 \mathrm{~mL})$ was stirred at $50{ }^{\circ} \mathrm{C}$ for $15 \mathrm{~h}$ under $\mathrm{N}_{2}$ atmosphere. The reaction mixture was poured into a mixed solvent of EtOAc and water. The aqueous layer was extracted with EtOAc (twice). The organic layers were combined and washed with water (three times). After dried over $\mathrm{Na}_{2} \mathrm{SO}_{4}$, the solvent was evaporated in vacuo to give a residue. The residue was purified by column chromatography $\left(\mathrm{SiO}_{2}\right.$, $\left.\mathrm{CHCl}_{3} / \mathrm{EtOAc}=5 / 1\right)$ and hexane/EtOAc $\left.(4: 1)\right)$ to afford $(R, R, R)-8(212 \mathrm{mg}, 238 \mu \mathrm{mol}, 92 \%)$ as a colorless powder.

mp $67-70{ }^{\circ} \mathrm{C} ;[\alpha]_{\mathrm{D}}^{23}=+215\left(\mathrm{CHCl}_{3}, c 0.10\right) ; \mathbf{I R}(\mathrm{KBr}) 3062,2955,2823,1622,1506,1471,1418,1238$, 1152, 1069, 1015, 978, 922, 808, $750 \mathrm{~cm}^{-1}$; ${ }^{1} \mathbf{H}$ NMR (400 MHz, $\left.\mathrm{CDCl}_{3}\right) \delta 0.65$ (t, $\left.J=7.4 \mathrm{~Hz}, 6 \mathrm{H}\right)$, 0.80-1.00 (m, 2H), 1.00-1.20 (m, 2H), 1.20-1.45 (m, 4H), $2.50(\mathrm{~s}, 6 \mathrm{H}), 3.30(\mathrm{~s}, 6 \mathrm{H}), 3.65-3.75(\mathrm{~m}, 2 \mathrm{H})$, $4.14(\mathrm{dt}, J=5.9,8.9 \mathrm{~Hz}, 2 \mathrm{H}), 4.97(\mathrm{AB} \mathrm{q}, \Delta v=12.7 \mathrm{~Hz}, J=5.5 \mathrm{~Hz}, 4 \mathrm{H}), 5.20(\mathrm{AB} \mathrm{q}, \Delta v=34.3 \mathrm{~Hz}, J=$ $6.7 \mathrm{~Hz}, 4 \mathrm{H}), 7.15-7.25(\mathrm{~m}, 4 \mathrm{H}), 7.27-7.37(\mathrm{~m}, 8 \mathrm{H}), 7.39(\mathrm{ddd}, J=1.9,6.1,8.1 \mathrm{~Hz}, 2 \mathrm{H}), 7.62(\mathrm{~d}, J=9.0 \mathrm{~Hz}$, $2 \mathrm{H}), 7.90(\mathrm{~d}, J=8.1 \mathrm{~Hz}, 2 \mathrm{H}), 7.99(\mathrm{~d}, J=9.0 \mathrm{~Hz}, 2 \mathrm{H}) ;{ }^{13} \mathbf{C} \mathbf{N M R}\left(100 \mathrm{MHz}, \mathrm{CDCl}_{3}\right) \delta 13.9,19.2,32.6$, 56.06, 56.09, 73.1, 95.8, 98.8, 117.7, 121.8, 124.3, 125.1, 125.2, 126.1, 126.4, 126.5, 127.0, 127.3, 127.9, 129.8, 130.0, 130.9, 131.5, 134.4, 148.2, 150.4, 153.1; HR MS (ESI $\left.{ }^{+}\right)$Calcd for $\mathrm{C}_{56} \mathrm{H}_{58} \mathrm{O}_{10} \mathrm{Na}: 913.3922$ $[\mathrm{M}+\mathrm{Na}]^{+}$. Found: 913.3887 . 


\section{Synthesis of $(R, S, R)-8$}

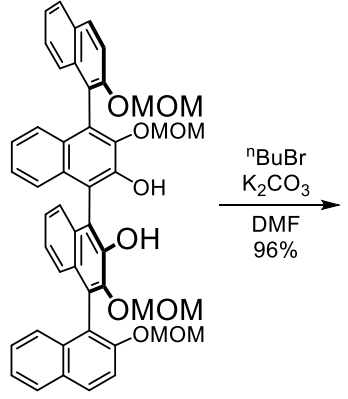

$(R, S, R)-7$

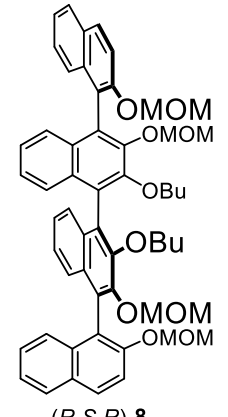

$(R, S, R)-8$

A mixture of $(R, S, R)-7$ (200 mg, $257 \mu \mathrm{mol}), \mathrm{n}-\mathrm{BuBr}(0.11 \mathrm{~mL}, 1.0 \mathrm{mmol})$, and $\mathrm{K}_{2} \mathrm{CO}_{3}(162 \mathrm{mg}, 1.17$ $\mathrm{mmol})$ in dry DMF $(0.5 \mathrm{~mL})$ was stirred at $50{ }^{\circ} \mathrm{C}$ for $14 \mathrm{~h}$ under $\mathrm{N}_{2}$ atmosphere. The reaction mixture was poured into a mixed solvent of EtOAc and water. The aqueous layer was extracted with EtOAc (twice). The organic layers were combined and washed with water (twice). After dried over $\mathrm{Na}_{2} \mathrm{SO}_{4}$, the solvent was evaporated in vacuo to give a residue. The residue was purified by column chromatography $\left(\mathrm{SiO}_{2}\right.$, hexane/EtOAc $=4 / 1)$ to afford $(R, S, R)-8(219 \mathrm{mg}, 246 \mu \mathrm{mol}, 96 \%)$ as a colorless powder.

mp 62-64 ${ }^{\circ} \mathrm{C} ;[\alpha]_{\mathrm{D}^{24}}=+44\left(\mathrm{CHCl}_{3}, c 0.10\right)$; IR (KBr) 3061, 2957, 2824, 1624, 1508, 1474, 1420, 1242, 1153, 1074, 1033, 978, 924, 808, $762 \mathrm{~cm}^{-1} ;{ }^{1} \mathbf{H}$ NMR (400 MHz, CDCl $) \delta 0.62$ (s, 6H), 0.99 (sext, $J=7.3$ Hz, 2H), 1.09 (sext, $J=7.1 \mathrm{~Hz}, 2 \mathrm{H}), 1.25-1.40(\mathrm{~m}, 4 \mathrm{H}), 2.59$ (s, 6H), $3.28(\mathrm{~s}, 6 \mathrm{H}), 3.81$ (td, $J=6.6,8.8 \mathrm{~Hz}$, $2 \mathrm{H}), 4.08(\mathrm{td}, J=6.3,8.8 \mathrm{~Hz}, 2 \mathrm{H}), 4.92(\mathrm{~d}, J=5.4 \mathrm{~Hz}, 2 \mathrm{H}), 5.04(\mathrm{~d}, J=5.5 \mathrm{~Hz}, 2 \mathrm{H}), 5.05(\mathrm{~d}, J=7.0 \mathrm{~Hz}$, 2H), $5.18(\mathrm{~d}, J=7.0 \mathrm{~Hz}, 2 \mathrm{H}), 7.19(\mathrm{ddd}, J=1.3,6.9,8.3 \mathrm{~Hz}, 2 \mathrm{H}), 7.23-7.28(\mathrm{~m}, 2 \mathrm{H}), 7.32(\mathrm{dd}, J=1.0,8.3$ $\mathrm{Hz}, 2 \mathrm{H}), 7.35-7.45(\mathrm{~m}, 10 \mathrm{H}), 7.88-7.93(\mathrm{~m}, 2 \mathrm{H}), 8.00(\mathrm{~d}, J=9.0 \mathrm{~Hz}, 2 \mathrm{H}) ;{ }^{13} \mathbf{C} \mathbf{N M R}\left(100 \mathrm{MHz}, \mathrm{CDCl}_{3}\right) \delta$ 13.8, 19.0, 32.3, 56.1, 56.2, 73.0, 95.3, 98.8, 117.0, 121.3, 124.3, 125.0, 125.3, 126.0, 126.2, 126.3, 126.7, 126.9, 127.4, 128.0, 129.8, 129.9, 130.9, 131.5, 134.3, 148.0, 150.2, 153.1; HR MS (ESI $\left.{ }^{+}\right)$Calcd for $\mathrm{C}_{56} \mathrm{H}_{58} \mathrm{ONa}$ : $913.3922[\mathrm{M}+\mathrm{Na}]^{+}$. Found: 913.3911.

\section{Synthesis of $(R, R, R)-9$}
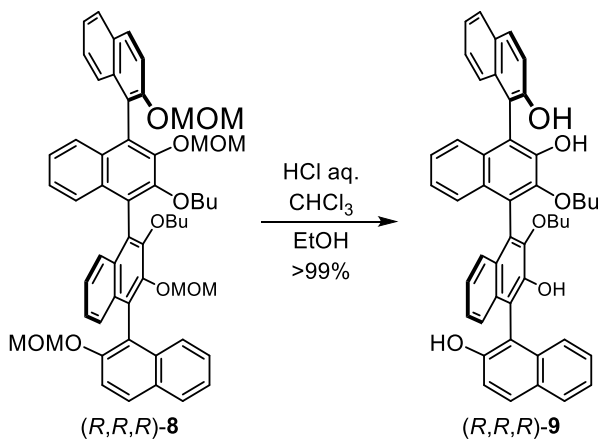

A solution of $(R, R, R)-\mathbf{8}(80.9 \mathrm{mg}, 90.8 \mu \mathrm{mol})$ in a mixed solvent of $6 \mathrm{M} \mathrm{HCl}$ aq. $(0.9 \mathrm{~mL}), \mathrm{CHCl}_{3}(9.0$ $\mathrm{mL})$, and $\mathrm{EtOH}(1.8 \mathrm{~mL})$ was stirred at $50{ }^{\circ} \mathrm{C}$ for $12 \mathrm{~h}$ under $\mathrm{N}_{2}$ atmosphere. Then water and $\mathrm{CHCl}_{3}$ was added. The organic layer was washed with water. After dried over $\mathrm{Na}_{2} \mathrm{SO}_{4}$, the solvent was evaporated to afford $(R, R, R)-9$ containing a little amount of water $(70.5 \mathrm{mg}, 98.6 \mu \mathrm{mol},>99 \%)$ as a yellow powder.

mp $136-140{ }^{\circ} \mathrm{C} ;[\alpha]_{\mathrm{D}}^{28}=+75\left(\mathrm{CHCl}_{3}, c 0.10\right)$; IR (KBr) 3059, 2957, 2932, 2870, 1622, 1508, 1466, 
1422, 1273, 1215, 1144, 968, 934, 818, $754 \mathrm{~cm}^{-1} ;{ }^{1} \mathbf{H}$ NMR $\left(400 \mathrm{MHz}, \mathrm{CDCl}_{3}\right) \delta 0.70(\mathrm{t}, J=7.4 \mathrm{~Hz}, 6 \mathrm{H})$, 1.04 (sext, $J=7.2 \mathrm{~Hz}, 2 \mathrm{H}$ ), 1.16 (sext, $J=7.0 \mathrm{~Hz}, 2 \mathrm{H}$ ), 1.46 (quin, $J=7.0 \mathrm{~Hz}, 4 \mathrm{H}$ ), 3.70 (dt, $J=6.6,9.2$ $\mathrm{Hz}, 2 \mathrm{H}), 3.88(\mathrm{dt}, J=6.3,9.3 \mathrm{~Hz}, 2 \mathrm{H}), 5.23(\mathrm{~s}, 2 \mathrm{H}), 6.14(\mathrm{~s}, 2 \mathrm{H}), 7.21(\mathrm{~d}, J=8.3 \mathrm{~Hz}, 2 \mathrm{H}), 7.25-7.35(\mathrm{~m}$, 8H), 7.38 (ddd, $J=1.1,6.8,8.0 \mathrm{~Hz}, 2 \mathrm{H}), 7.42-7.50(\mathrm{~m}, 4 \mathrm{H}), 7.93(\mathrm{~d}, J=8.0 \mathrm{~Hz}, 2 \mathrm{H}), 8.01(\mathrm{~d}, J=8.9 \mathrm{~Hz}$, 2H); ${ }^{13} \mathbf{C}$ NMR $\left(100 \mathrm{MHz}, \mathrm{CDCl}_{3}\right) \delta 13.8,19.1,32.3,74.0,113.2,113.4,117.9,123.8,124.5,124.96$, $125.04,125.3,126.45,126.54,127.0,128.6,129.4,129.6,130.7,130.9,133.5,145.9,147.6,152.0$; HR MS $\left(\mathrm{ESI}^{+}\right)$Calcd for $\mathrm{C}_{48} \mathrm{H}_{42} \mathrm{O}_{6} \mathrm{Na}: 737.2874[\mathrm{M}+\mathrm{Na}]^{+}$. Found: 737.2887 .

\section{Synthesis of $(R, S, R)-9$}
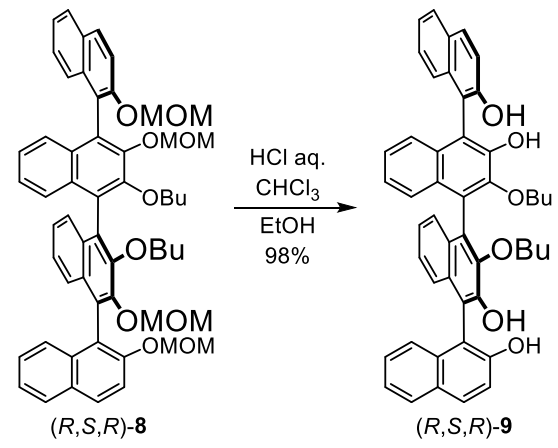

A solution of $(R, S, R)-8(100 \mathrm{mg}, 112 \mu \mathrm{mol})$ in a mixed solvent of $6 \mathrm{M} \mathrm{HCl}$ aq. $(1.1 \mathrm{~mL}), \mathrm{CHCl}_{3}(11 \mathrm{~mL})$, and $\mathrm{EtOH}(2.2 \mathrm{~mL})$ was stirred at $50{ }^{\circ} \mathrm{C}$ for $17 \mathrm{~h}$ under $\mathrm{N}_{2}$ atmosphere. Then water and $\mathrm{CHCl}_{3}$ was added. The organic layer was washed with water (twice). After dried over $\mathrm{Na}_{2} \mathrm{SO}_{4}$, the solvent was evaporated to afford $(R, S, R)-9(78.6 \mathrm{mg}, 110 \mu \mathrm{mol}, 98 \%)$ as a colorless powder.

mp $110-112{ }^{\circ} \mathrm{C} ;[\alpha]_{\mathrm{D}} 22=-14\left(\mathrm{CHCl}_{3}, c 0.11\right)$; IR (KBr) 3528, 3059, 2959, 2928, 2859, 1713, 1622 , 1508, 1466, 1423, 1385, 1086, 974, 935, 862, 816, $760 \mathrm{~cm}^{-1} ;{ }^{1} \mathbf{H}$ NMR $\left(400 \mathrm{MHz}, \mathrm{CDCl}_{3}\right) \delta 0.64(\mathrm{t}, J=7.4$ $\mathrm{Hz}, 6 \mathrm{H}$ ), 0.90 (sext, $J=7.1 \mathrm{~Hz}, 2 \mathrm{H}), 1.07$ (sext, $J=7.0 \mathrm{~Hz}, 2 \mathrm{H}), 1.25-1.45(\mathrm{~m}, 4 \mathrm{H}), 3.69$ (dt, $J=6.6,9.2$ $\mathrm{Hz}, 2 \mathrm{H}), 3.91$ (dt, $J=6.1,9.3 \mathrm{~Hz}, 2 \mathrm{H}), 5.02(\mathrm{~s}, 2 \mathrm{H}), 6.05(\mathrm{~s}, 2 \mathrm{H}), 7.25-7.40(\mathrm{~m}, 8 \mathrm{H}), 7.40-7.50(\mathrm{~m}, 8 \mathrm{H})$, 7.90-7.97 (m, 2H), $8.01(\mathrm{~d}, J=8.9 \mathrm{~Hz}, 2 \mathrm{H}) ;{ }^{13} \mathbf{C} \mathbf{~ N M R}\left(100 \mathrm{MHz}, \mathrm{CDCl}_{3}\right) \delta 13.5,19.0,32.2,73.9,113.0$, 113.3, 117.8, 123.9, 124.7, 125.0, 125.1, 125.7, 126.4, 126.6, 127.2, 128.6, 129.4, 129.5, 130.7, 131.0, 133.5, 145.7, 147.6, 151.9; HR MS (ESI ${ }^{+}$Calcd for $\mathrm{C}_{48} \mathrm{H}_{42} \mathrm{O}_{6} \mathrm{Na}$ : 737.2874 [M+Na] $]^{+}$. Found: 737.2897. 


\section{Synthesis of $(R)-2$}

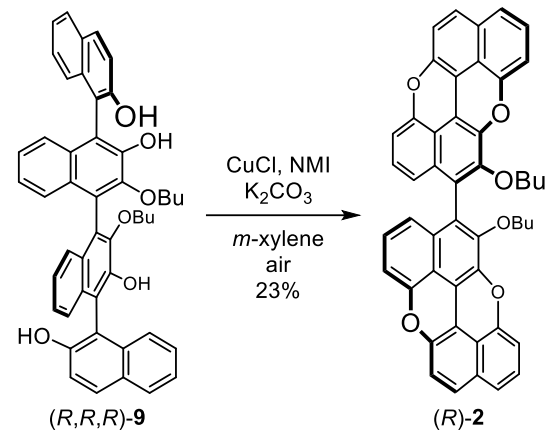

A mixture of $(R, R, R)-9(227 \mathrm{mg}, 318 \mu \mathrm{mol}), \mathrm{CuCl}(269 \mathrm{mg}, 2.72 \mathrm{mmol}), \mathrm{K}_{2} \mathrm{CO}_{3}(266 \mathrm{mg}, 1.92 \mathrm{mmol})$, and $\mathrm{N}$-methylimidazole $(0.20 \mathrm{~mL}, 2.5 \mathrm{mmol})$ in $\mathrm{m}$-xylene $(3.2 \mathrm{~mL})$ was stirred at $120{ }^{\circ} \mathrm{C}$ for $20 \mathrm{~h}$ under air. The mixture was cooled and diluted with $\mathrm{CHCl}_{3}$. The product was purified by column chromatography $\left(\mathrm{SiO}_{2}, \mathrm{CHCl}_{3} /\right.$ hexane $\left.=1 / 1\right)$ and recrystallization from $\mathrm{CHCl}_{3} /$ hexane to afford $(R)-2(51.3 \mathrm{mg}, 72.6 \mu \mathrm{mol}$, $23 \%$ ) as a yellow powder.

mp 238-242 ${ }^{\circ} \mathrm{C} ;[\alpha]_{\mathrm{D}}{ }^{18}=-13\left(\mathrm{CHCl}_{3}, c\right.$ 0.10); IR (KBr) 3051, 2953, 2932, 2868, 1626, 1499, 1423, 1327, 1246, 1167, 1152, 1074, 993, 818, $761 \mathrm{~cm}^{-1} ;{ }^{1} \mathbf{H} \mathbf{N M R}\left(400 \mathrm{MHz}, \mathrm{CDCl}_{3}\right) \delta 0.72(\mathrm{t}, J=7.4 \mathrm{~Hz}, 6 \mathrm{H})$, $1.05-1.25(\mathrm{~m}, 4 \mathrm{H}), 1.45$ (quin, $J=7.1 \mathrm{~Hz}, 4 \mathrm{H}), 3.85(\mathrm{dt}, J=6.4,9.3 \mathrm{~Hz}, 2 \mathrm{H}), 4.05(\mathrm{dt}, J=6.4,9.2 \mathrm{~Hz}, 2 \mathrm{H})$, $6.54(\mathrm{~d}, J=8.9 \mathrm{~Hz}, 2 \mathrm{H}), 6.61(\mathrm{~d}, J=7.8 \mathrm{~Hz}, 2 \mathrm{H}), 6.73(\mathrm{dd}, J=2.8,5.7 \mathrm{~Hz}, 2 \mathrm{H}), 6.95(\mathrm{~d}, J=7.9 \mathrm{~Hz}, 2 \mathrm{H})$, $6.99(\mathrm{~d}, J=9.2 \mathrm{~Hz}, 2 \mathrm{H}), 7.10-7.18(\mathrm{~m}, 4 \mathrm{H}), 7.37(\mathrm{~d}, J=9.3 \mathrm{~Hz}, 2 \mathrm{H}) ;{ }^{13} \mathbf{C ~ N M R}\left(100 \mathrm{MHz}, \mathrm{CDCl}_{3}\right) \delta 13.8$, 18.9, 32.2, 73.6, 107.8, 109.1, 111.5, 113.2, 117.5, 118.1, 118.5, 120.4, 121.6, 123.7, 126.7, 127.2, 127.8, 131.4, 131.8, 139.0, 144.4, 146.0, 152.4, 152.7; HR MS (ESI $\left.{ }^{+}\right)$Calcd for $\mathrm{C}_{48} \mathrm{H}_{34} \mathrm{O}_{6} \mathrm{Na}: 729.2248[\mathrm{M}+\mathrm{Na}]^{+}$. Found: 729.2224.

\section{Synthesis of $(S)-2$}

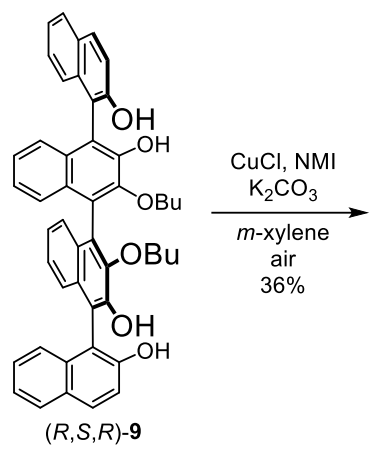

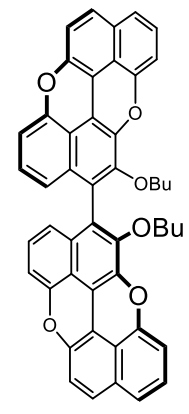

$(S)-2$

A mixture of $(R, S, R)-9$ (182 mg, $255 \mu \mathrm{mol}), \mathrm{CuCl}(204 \mathrm{mg}, 2.06 \mathrm{mmol}), \mathrm{K}_{2} \mathrm{CO}_{3}(213 \mathrm{mg}, 1.54 \mathrm{mmol})$, and $N$-methylimidazole $(0.16 \mathrm{~mL}, 2.0 \mathrm{mmol})$ in $m$-xylene $(2.5 \mathrm{~mL})$ was stirred at $120{ }^{\circ} \mathrm{C}$ for $20 \mathrm{~h}$ under air. The mixture was cooled and diluted with $\mathrm{CHCl}_{3}$. The product was purified by column chromatography $\left(\mathrm{SiO}_{2}, \mathrm{CHCl}_{3} /\right.$ hexane $\left.=1 / 1\right)$ and recrystallization from $\mathrm{CHCl}_{3} /$ hexane to afford $(S)-2(65.3 \mathrm{mg}, 92.4 \mu \mathrm{mol}$, $36 \%$ ) as a yellow powder. The NMR data were in agreement with those of $(R)-2$. 


\section{Synthesis of $(R, R, R)-14$}
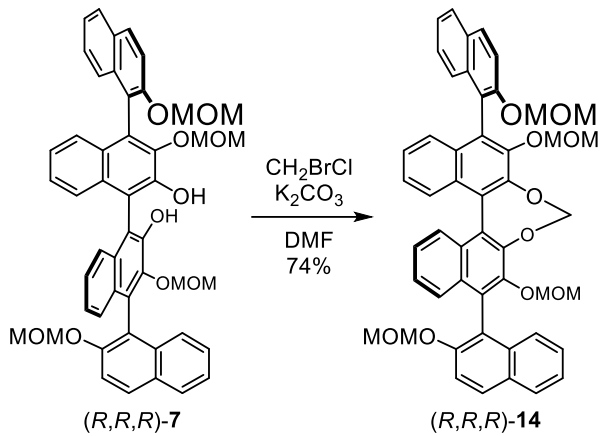

A mixture of $(R, R, R)-7(501 \mathrm{mg}, 643 \mu \mathrm{mol}), \mathrm{CH}_{2} \mathrm{BrCl}(1.30 \mathrm{~mL}, 19.4 \mathrm{mmol})$, and $\mathrm{K}_{2} \mathrm{CO}_{3}(891 \mathrm{mg}, 6.45$ mmol) in dry DMF $(22 \mathrm{~mL})$ was stirred at $50{ }^{\circ} \mathrm{C}$ for $15 \mathrm{~h}$ under $\mathrm{N}_{2}$ atmosphere. The reaction mixture was poured into water and EtOAc. The aqueous layer was extracted with EtOAc. The organic layers were combined and washed with water (three times). After dried over $\mathrm{Na}_{2} \mathrm{SO}_{4}$, the solvent was evaporated in vacuo to give a residue. The residue was purified by column chromatography $\left(\mathrm{SiO}_{2}, \mathrm{CHCl}_{3} /\right.$ hexane/EtOAc $=20 / 5 / 1)$ to afford $(R, R, R)-\mathbf{1 4}(374 \mathrm{mg}, 473 \mathrm{mmol}, 74 \%)$ as a colorless powder.

mp $128-132{ }^{\circ} \mathrm{C} ;[\alpha]_{\mathrm{D}}{ }^{28}=-28\left(\mathrm{CHCl}_{3}, c 0.10\right)$; IR (KBr) 3061, 2957, 2903, 2826, 1622, 1508, 1472, 1418, 1379, 1350, 1260, 1153, 1049, 970, 922, 812, $760 \mathrm{~cm}^{-1}$; ${ }^{1} \mathbf{H}$ NMR $\left(400 \mathrm{MHz}, \mathrm{CDCl}_{3}\right) \delta 2.92(\mathrm{~s}, 6 \mathrm{H})$, $3.31(\mathrm{~s}, 6 \mathrm{H}), 4.95(\mathrm{AB} \mathrm{q}, \Delta v=29.7 \mathrm{~Hz}, J=5.6 \mathrm{~Hz}, 4 \mathrm{H}), 5.19(\mathrm{AB} \mathrm{q}, \Delta v=22.6 \mathrm{~Hz}, J=6.8 \mathrm{~Hz}, 4 \mathrm{H}), 5.88(\mathrm{~s}$, 2H), 7.20-7.30 (m, 4H), 7.32-7.38 (m, 6H), 7.41 (ddd, $J=2.4,5.7,8.0 \mathrm{~Hz}, 2 \mathrm{H}), 7.65$ (d, $J=9.1 \mathrm{~Hz}, 2 \mathrm{H}$ ), $7.71(\mathrm{~d}, J=8.0 \mathrm{~Hz}, 2 \mathrm{H}), 7.91(\mathrm{~d}, J=8.0 \mathrm{~Hz}, 2 \mathrm{H}), 8.01(\mathrm{~d}, J=9.1 \mathrm{~Hz}, 2 \mathrm{H}) ;{ }^{13} \mathbf{C} \mathbf{N M R}\left(100 \mathrm{MHz}, \mathrm{CDCl}_{3}\right) \delta$ 56.2, 56.5, 95.3, 99.3, 103.2, 116.8, 120.7, 124.4, 125.1, 125.6, 125.8, 126.6, 127.0, 127.2, 128.1, 128.2, 128.4, 129.6, 129.8, 130.1, 131.9, 134.2, 145.8, 146.8, 153.0; HR MS $\left(\mathrm{ESI}^{+}\right)$Calcd for $\mathrm{C}_{49} \mathrm{H}_{42} \mathrm{O}_{10} \mathrm{Na}$ : $813.2670[\mathrm{M}+\mathrm{Na}]^{+}$. Found: 813.2639.

\section{Synthesis of $(R, R, R)-15$}
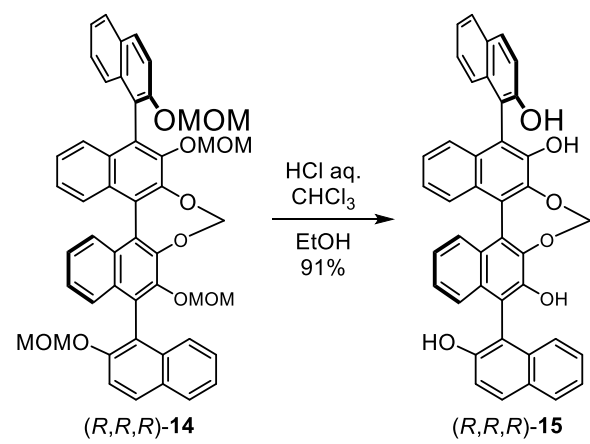

A solution of $(R, R, R)-\mathbf{1 4}(374 \mathrm{mg}, 473 \mu \mathrm{mol})$ in a mixed solvent of $6 \mathrm{M} \mathrm{HCl}$ aq. $(4.7 \mathrm{~mL}), \mathrm{CHCl}_{3}(47$ $\mathrm{mL}$ ), and $\mathrm{EtOH}(9.5 \mathrm{~mL})$ was stirred at $50{ }^{\circ} \mathrm{C}$ for $18 \mathrm{~h}$ under $\mathrm{N}_{2}$ atmosphere. Then water and $\mathrm{CHCl}_{3}$ was added. The organic layer was washed with water (twice). After dried over $\mathrm{Na}_{2} \mathrm{SO}_{4}$, the solvent was evaporated to afford $(R, R, R)-\mathbf{1 5}(264 \mathrm{mg}, 430 \mu \mathrm{mol}, 91 \%)$ as a colorless powder.

mp 223-226 ${ }^{\circ} \mathrm{C} ;[\alpha]_{\mathrm{D}}{ }^{20}=-98\left(\mathrm{CHCl}_{3}, c 0.10\right)$; IR (KBr) 3061, 2926, 1686, 1578, 1508, 1416, 1352, 1269, 1136, 1088, 1013, 926, 818, $758 \mathrm{~cm}^{-1} ;{ }^{1} \mathbf{H}$ NMR (400 MHz, $\left.\mathrm{CDCl}_{3}\right) \delta 5.16$ (s, 2H), $5.81(\mathrm{~s}, 2 \mathrm{H}), 5.93$ 
(s, 2H), 7.20-7.45 (m, 12H), 7.46 (d, $J=8.8 \mathrm{~Hz}, 2 \mathrm{H}), 7.73$ (dd, $J=1.7,7.4 \mathrm{~Hz}, 2 \mathrm{H}), 7.94$ (dd, $J=1.8,7.5$ $\mathrm{Hz}, 2 \mathrm{H}), 8.03(\mathrm{~d}, J=8.9 \mathrm{~Hz}, 2 \mathrm{H}) ;{ }^{13} \mathbf{C}$ NMR $\left(100 \mathrm{MHz}, \mathrm{CDCl}_{3}\right) \delta 103.2,112.6,114.6,117.9,124.0,124.6$, 124.8, 125.4, 127.0, 127.4, 127.5, 127.7, 128.0, 128.6, 129.5, 131.2, 131.8, 133.5, 141.0, 146.2, 152.1; HR MS $\left(\mathrm{ESI}^{+}\right)$Calcd for $\mathrm{C}_{41} \mathrm{H}_{26} \mathrm{O}_{6} \mathrm{Na}: 637.1622[\mathrm{M}+\mathrm{Na}]^{+}$. Found: 637.1596.

\section{Synthesis of $(R)-3$}
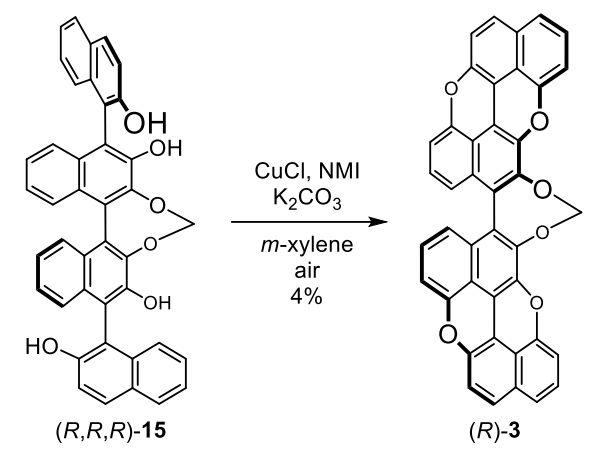

A mixture of $(R, R, R)-\mathbf{1 5}(264 \mathrm{mg}, 429 \mu \mathrm{mol}), \mathrm{CuCl}(341 \mathrm{mg}, 3.44 \mathrm{mmol}), \mathrm{K}_{2} \mathrm{CO}_{3}(364 \mathrm{mg}, 2.63 \mathrm{mmol})$, and $N$-methylimidazole $(0.27 \mathrm{~mL}, 3.4 \mathrm{mmol})$ in $m$-xylene $(4.3 \mathrm{~mL})$ was stirred at $120{ }^{\circ} \mathrm{C}$ for $20 \mathrm{~h}$ under air. The mixture was cooled and diluted with $\mathrm{CHCl}_{3}$. The product was purified by column chromatography $\left(\mathrm{SiO}_{2}, \mathrm{CHCl}_{3} /\right.$ hexane $\left.=1 / 1\right)$ and recrystallization from $\mathrm{CHCl}_{3} /$ hexane to afford $(R)-\mathbf{3}(9.6 \mathrm{mg}, 0.016 \mathrm{mmol}$, $4 \%)$ as an orange powder.

$\mathbf{m p}>300{ }^{\circ} \mathrm{C} ;[\alpha]_{\mathrm{D}}{ }^{17}=-800\left(\mathrm{CHCl}_{3}, c 0.010\right) ; \mathbf{I R}(\mathrm{KBr}) 3053,2920,2851,1626,1501,1411,1335,1246$, 1140, 1069, 989, 930, 818, $750 \mathrm{~cm}^{-1} ;{ }^{1} \mathbf{H}$ NMR $\left(400 \mathrm{MHz}, \mathrm{CDCl}_{3}\right) \delta 5.76(\mathrm{~s}, 2 \mathrm{H}), 6.70(\mathrm{~d}, J=7.6 \mathrm{~Hz}, 2 \mathrm{H})$, $6.76(\mathrm{~d}, J=8.6 \mathrm{~Hz}, 2 \mathrm{H}), 6.81(\mathrm{dd}, J=2.7,5.7 \mathrm{~Hz}, 2 \mathrm{H}) 6.98-7.01(\mathrm{~m}, 4 \mathrm{H}), 7.15-7.20(\mathrm{~m}, 4 \mathrm{H}), 7.39(\mathrm{~d}, J=$ $9.0 \mathrm{~Hz}, 2 \mathrm{H})$; HR MS (APCI- $)$ Calcd for $\mathrm{C}_{41} \mathrm{H}_{18} \mathrm{O}_{6}: 606.1109$ [M]- . Found: 606.1100.

\section{Synthesis of $(R, R)-4$}

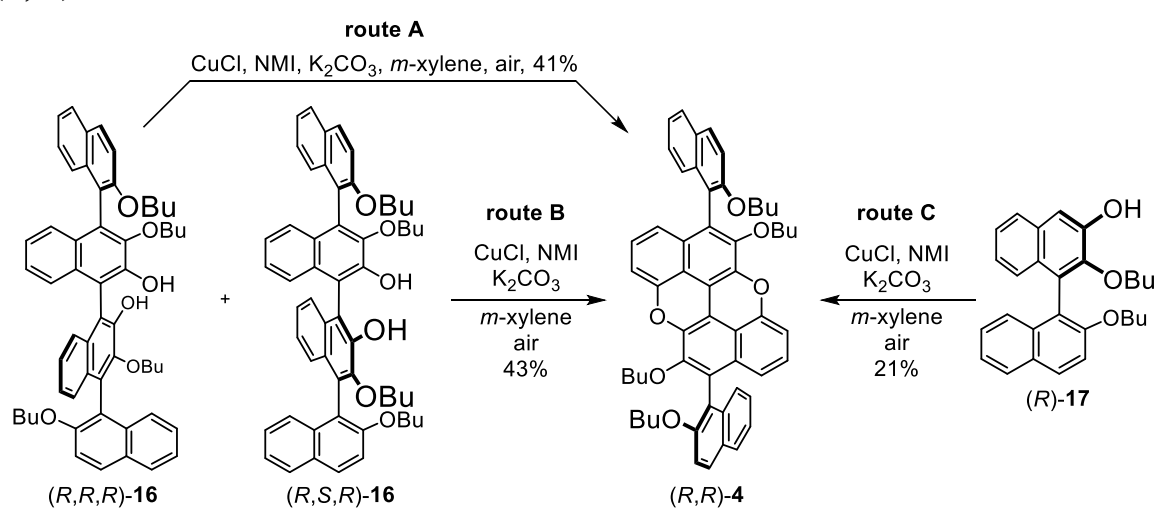

route A; A mixture of $(R, R, R)-\mathbf{1 6}(106 \mathrm{mg}, 128 \mu \mathrm{mol}), \mathrm{CuCl}(49.1 \mathrm{mg}, 496 \mu \mathrm{mol}), \mathrm{K}_{2} \mathrm{CO}_{3}(53.4 \mathrm{mg}, 386$ $\mu \mathrm{mol}$ ), and $N$-methylimidazole ( $38 \mu \mathrm{L}, 0.48 \mathrm{mmol})$ in $m$-xylene $\left(0.6 \mathrm{~mL}\right.$ ) was stirred at $120{ }^{\circ} \mathrm{C}$ for $24 \mathrm{~h}$ under air. The mixture was cooled, diluted with water, and extracted with $\mathrm{CHCl}_{3}$ (three times). The organic layer was washed with water (twice). After dried over $\mathrm{Na}_{2} \mathrm{SO}_{4}$, the solvent was evaporated to give a residue. 
The residue was purified by column chromatography $\left(\mathrm{SiO}_{2}, \mathrm{CHCl}_{3} /\right.$ hexane $\left.=1 / 1\right)$ to afford $(R, R)-\mathbf{4}(42.8$ $\mathrm{mg}, 52.0 \mu \mathrm{mol}, 41 \%)$ as a yellow powder.

route B; A mixture of $(R, S, R)-\mathbf{1 6}(106 \mathrm{mg}, 128 \mu \mathrm{mol}), \mathrm{CuCl}(49.4 \mathrm{mg}, 499 \mu \mathrm{mol}), \mathrm{K}_{2} \mathrm{CO}_{3}(54.0 \mathrm{mg}, 391$ $\mu \mathrm{mol})$, and $N$-methylimidazole $(38 \mu \mathrm{L}, 0.48 \mathrm{mmol})$ in $m$-xylene $\left(0.6 \mathrm{~mL}\right.$ ) was stirred at $120{ }^{\circ} \mathrm{C}$ for $24 \mathrm{~h}$ under air. The mixture was cooled, diluted with water and extracted with $\mathrm{CHCl}_{3}$ (three times). The organic layer was washed with water (twice). After dried over $\mathrm{Na}_{2} \mathrm{SO}_{4}$, the solvent was evaporated to give a residue. The residue was purified by column chromatography $\left(\mathrm{SiO}_{2}, \mathrm{CHCl}_{3} /\right.$ hexane $\left.=1 / 1\right)$ to afford $(R, R)-4(45.5$ $\mathrm{mg}, 55.3 \mu \mathrm{mol}, 43 \%)$ as a yellow powder.

route $\mathbf{C}$; A mixture of $(R)-\mathbf{1 7}(50.2 \mathrm{mg}, 121 \mu \mathrm{mol}), \mathrm{CuCl}(27.3 \mathrm{mg}, 276 \mu \mathrm{mol}), \mathrm{K}_{2} \mathrm{CO}_{3}(29.4 \mathrm{mg}, 213$ $\mu \mathrm{mol})$, and $N$-methylimidazole $(19 \mu \mathrm{L}, 0.24 \mathrm{mmol})$ in $m$-xylene $(0.3 \mathrm{~mL})$ was stirred at $120{ }^{\circ} \mathrm{C}$ for $20 \mathrm{~h}$ under air. The mixture was cooled, diluted with water and extracted with $\mathrm{CHCl}_{3}$ (three times). The organic layer was washed with water and $1 \mathrm{M} \mathrm{HCl}$ aq. (twice). After dried over $\mathrm{Na}_{2} \mathrm{SO}_{4}$, the solvent was evaporated to give a residue. The residue was purified by column chromatography $\left(\mathrm{SiO}_{2}, \mathrm{CHCl}_{3} /\right.$ hexane $\left.=2 / 1\right)$ and PTLC $\left(\mathrm{CHCl}_{3} /\right.$ hexane (3:1)) to afford $(R, R)-\mathbf{4}(10.5 \mathrm{mg}, 12.8 \mu \mathrm{mol}, 21 \%)$ as a yellow powder.

mp 63-66 ${ }^{\circ} \mathrm{C} ;[\alpha]_{\mathrm{D}^{20}}=+172\left(\mathrm{CHCl}_{3}, c 0.10\right)$; IR (KBr) 3057, 2959, 2926, 2855, 1624, 1508, 1423, 1367 , 1261, 1213, 1070, 802, $746 \mathrm{~cm}^{-1} ;{ }^{1} \mathbf{H}$ NMR $\left(400 \mathrm{MHz}, \mathrm{CDCl}_{3}\right) \delta 0.59(\mathrm{t}, J=7.4 \mathrm{~Hz}, 6 \mathrm{H}), 0.88(\mathrm{t}, J=7.4$ $\mathrm{Hz}, 6 \mathrm{H}), 0.83-0.98(\mathrm{~m}, 4 \mathrm{H}), 1.18-1.35(\mathrm{~m}, 8 \mathrm{H}), 1.52-1.62(\mathrm{~m}, 4 \mathrm{H}), 3.79(\mathrm{dt}, J=6.4,9.2 \mathrm{~Hz}, 2 \mathrm{H}), 3.89(\mathrm{dt}$, $J=6.5,9.3 \mathrm{~Hz}, 2 \mathrm{H}), 4.02(\mathrm{dt}, J=6.6,9.4 \mathrm{~Hz}, 2 \mathrm{H}), 4.09(\mathrm{dt}, J=6.5,9.5 \mathrm{~Hz}, 2 \mathrm{H}), 6.41(\mathrm{~d}, J=8.1 \mathrm{~Hz}, 2 \mathrm{H})$, $6.65(\mathrm{~d}, J=7.2 \mathrm{~Hz}, 2 \mathrm{H}), 6.89(\mathrm{t}, J=8.1 \mathrm{~Hz}, 2 \mathrm{H}), 7.28-7.38(\mathrm{~m}, 6 \mathrm{H}), 7.41(\mathrm{~d}, J=9.0 \mathrm{~Hz}, 2 \mathrm{H}), 7.85(\mathrm{dd}, J=$ 2.3, $6.7 \mathrm{~Hz}, 2 \mathrm{H}), 7.93(\mathrm{~d}, J=9.0 \mathrm{~Hz}, 2 \mathrm{H}) ;{ }^{13} \mathbf{C} \mathbf{N M R}\left(100 \mathrm{MHz}, \mathrm{CDCl}_{3}\right) \delta 13.7,13.8,18.6,19.1,31.6,32.1$, 69.2, 73.4, 107.9, 112.9, 115.1, 118.1, 119.0, 119.1, 119.2, 123.6, 125.3, 126.6, 127.3, 128.0, 129.2, 129.6, 132.2, 133.9, 139.2, 152.5, 154.5; HR MS (ESI+) Calcd for $\mathrm{C}_{56} \mathrm{H}_{54} \mathrm{O}_{6}: 822.3915$ [M] ${ }^{+}$. Found: 822.3902.

\section{Synthesis of $(R)-10$}

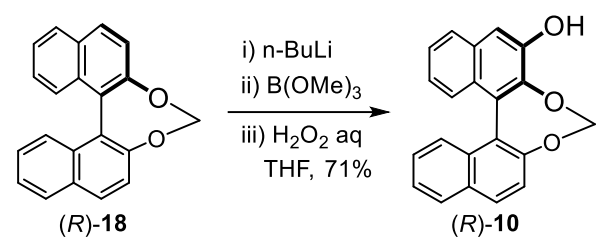

To a solution of $(R)-\mathbf{1 8}(1.52 \mathrm{~g}, 5.08 \mathrm{mmol})$ in dry THF $(15 \mathrm{~mL}), 1.6 \mathrm{M} \mathrm{n}-\mathrm{BuLi}$ in hexane $(4.9 \mathrm{~mL}, 7.8$ mmol) was added dropwise at $-78{ }^{\circ} \mathrm{C}$ under $\mathrm{N}_{2}$ atmosphere. The solution was stirred at $0{ }^{\circ} \mathrm{C}$ for $1 \mathrm{~h}$. $\mathrm{B}(\mathrm{OMe})_{3}(1.20 \mathrm{~mL}, 10.8 \mathrm{mmol})$ was added to the solution at $-78^{\circ} \mathrm{C}$, and the reaction mixture was stirred at room temperature for $18 \mathrm{~h}$. After removal of organic solvent in vacuo, $\mathrm{CHCl}_{3}$ and $\mathrm{H}_{2} \mathrm{O}_{2}(30 \mathrm{wt} \%$ solution in water, $1.60 \mathrm{~mL}$ ) was added at $0{ }^{\circ} \mathrm{C}$, and the solution was stirred at $50{ }^{\circ} \mathrm{C}$ for $4 \mathrm{~h}$. Then saturated aqueous $\mathrm{Na}_{2} \mathrm{SO}_{3}$ and $\mathrm{CHCl}_{3}$ were added. The organic layer was separated and washed with brine. After dried over $\mathrm{Na}_{2} \mathrm{SO}_{4}$, the solvent was evaporated in vacuo to give a residue. The residue was purified by column chromatography $\left(\mathrm{SiO}_{2}, \mathrm{CHCl}_{3}\right)$ to afford $(R)-\mathbf{1 0}(1.08 \mathrm{~g}, 3.44 \mathrm{mmol}, 71 \%)$ as a reddish brown powder.

mp 83-86 ${ }^{\circ} \mathrm{C} ;[\alpha]_{\mathrm{D}}^{20}=-643\left(\mathrm{CHCl}_{3}, c 0.10\right)$; IR (KBr) 3443, 3051, 2970, 2901, 1628, 1508, 1449, 1333, 
$1267,1242,1215,1065,988,947,878,829,752 \mathrm{~cm}^{-1} ;{ }^{1} \mathbf{H}$ NMR $\left(400 \mathrm{MHz}, \mathrm{CDCl}_{3}\right) \delta 5.74(\mathrm{AB} \mathrm{q}, \Delta v=$ $7.6 \mathrm{~Hz}, J=3.1 \mathrm{~Hz}, 2 \mathrm{H}), 5.91(\mathrm{~s}, 1 \mathrm{H}), 7.16$ (ddd, $J=1.4,6.9,8.4 \mathrm{~Hz}, 1 \mathrm{H}), 7.33$ (ddd, $J=1.4,6.9,8.5 \mathrm{~Hz}$, 1H), 7.35-7.44 (m, 4H), 7.50 (s, 1H), $7.54(\mathrm{~d}, J=8.6 \mathrm{~Hz}, 1 \mathrm{H}), 7.81(\mathrm{~d}, J=8.7 \mathrm{~Hz}, 1 \mathrm{H}), 7.94(\mathrm{~d}, J=8.2 \mathrm{~Hz}$, $1 \mathrm{H}), 8.00(\mathrm{~d}, J=8.7 \mathrm{~Hz}, 1 \mathrm{H}) ;{ }^{13} \mathbf{C}$ NMR $\left(100 \mathrm{MHz}, \mathrm{CDCl}_{3}\right) \delta 103.0,111.2,120.7,123.8,125.4,125.8$, $126.1,126.3,126.4,126.9,127.05,127.13,127.3,128.9,130.8,132.0,132.2,132.6,140.5,147.0,151.2$; HR MS (ESI-) Calcd for $\mathrm{C}_{21} \mathrm{H}_{13} \mathrm{O}_{3}: 313.0865[\mathrm{M}-\mathrm{H}]^{-}$. Found: 313.0866.

\section{Synthesis of $(R, R, R)-11$ and $(R, S, R)-11$}

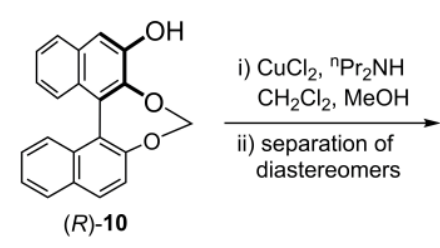

(R)-10

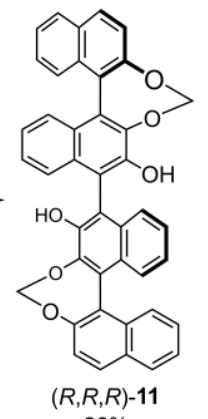

$26 \%$

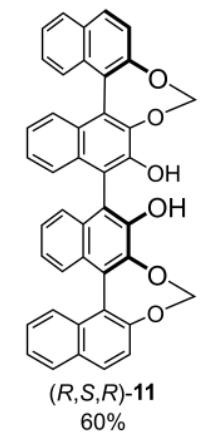

$60 \%$

To a mixture of $\mathrm{CuCl}_{2}(17.4 \mathrm{~g}, 129 \mathrm{mmol})$ in dry $\mathrm{MeOH}(240 \mathrm{~mL}), \mathrm{n}-\mathrm{Pr}_{2} \mathrm{NH}(24.0 \mathrm{~mL}, 176 \mathrm{mmol})$ was added under $\mathrm{N}_{2}$ atmosphere with ice-bath cooling. After $2.5 \mathrm{~h}$, a solution of $(R)-\mathbf{1 0}(4.05 \mathrm{~g}, 12.9 \mathrm{mmol})$ in dry $\mathrm{CH}_{2} \mathrm{Cl}_{2}(240 \mathrm{~mL})$ was added, and the reaction mixture was stirred at $0{ }^{\circ} \mathrm{C}$ for $16 \mathrm{~h}$. The reaction mixture was poured into a mixed solvent of $1 \mathrm{M} \mathrm{HCl}$ aq. and $\mathrm{CHCl}_{3}$. The aqueous layer was extracted with $\mathrm{CHCl}_{3}$. The organic layers were combined, washed with brine, dried over $\mathrm{Na}_{2} \mathrm{SO}_{4}$, and evaporated to give a residue. The residue was purified by column chromatography $\left(\mathrm{SiO}_{2}\right.$, hexane/CHCl $\left.3 / \mathrm{EtOAc}=2 / 2 / 1\right)$ to afford $(R, R, R)-\mathbf{1 1}(1.05 \mathrm{~g}, 1.68 \mathrm{mmol}, 26 \%)$ as a yellow powder and $(R, S, R)-\mathbf{1 1}(2.43 \mathrm{~g}, 3.88 \mathrm{mmol}, 60 \%)$ as a yellow powder.

$(R, R, R)-11: \mathbf{m p} 289-291{ }^{\circ} \mathrm{C} ;[\alpha]_{\mathrm{D}}^{20}=-411\left(\mathrm{CHCl}_{3}, c\right.$ 0.10); IR $(\mathrm{KBr}) 3357,3065,2967,2905,1622$, 1510, 1439, 1425, 1402, 1329, 1211, 1109, 986, 949, 831, $758 \mathrm{~cm}^{-1}$; ${ }^{1} \mathbf{H}$ NMR $\left(400 \mathrm{MHz}, \mathrm{CDCl}_{3}\right) \delta 5.85$ $(\mathrm{AB} \mathrm{q}, \Delta v=8.6 \mathrm{~Hz}, J=3.2 \mathrm{~Hz}, 4 \mathrm{H}), 5.97(\mathrm{~s}, 2 \mathrm{H}), 7.21$ (ddd, $J=1.4,6.8,8.4 \mathrm{~Hz}, 2 \mathrm{H}), 7.31$ (ddd, $J=1.4$, 6.8, 8.4 Hz, 2H), $7.38(\mathrm{ddd}, J=1.3,6.9,8.5 \mathrm{~Hz}, 2 \mathrm{H}), 7.44(\mathrm{~d}, J=8.5 \mathrm{~Hz}, 2 \mathrm{H}), 7.51(\mathrm{ddd}, J=1.1,7.0,8.2$ $\mathrm{Hz}, 2 \mathrm{H}), 7.56(\mathrm{~d}, J=8.7 \mathrm{~Hz}, 2 \mathrm{H}), 7.58(\mathrm{~d}, J=8.6 \mathrm{~Hz}, 2 \mathrm{H}), 7.74(\mathrm{~d}, J=8.5 \mathrm{~Hz}, 2 \mathrm{H}), 7.99(\mathrm{~d}, J=8.1 \mathrm{~Hz}$, $2 \mathrm{H}), 8.06(\mathrm{~d}, J=8.7 \mathrm{~Hz}, 2 \mathrm{H}) ;{ }^{13} \mathbf{C}$ NMR $\left(100 \mathrm{MHz}, \mathrm{CDCl}_{3}\right) \delta 103.2,115.9,120.8,124.1,125.5,125.6$, 126.1, 126.3, 126.4, 127.1, 127.27, 127.34, 127.4, 128.6, 130.9, 131.8, 132.1, 132.4, 140.6, 145.4, 151.5; HR MS (ESI $\left.{ }^{+}\right)$Calcd for $\mathrm{C}_{42} \mathrm{H}_{26} \mathrm{O}_{6} \mathrm{Na}: 649.1622[\mathrm{M}+\mathrm{Na}]^{+}$. Found: 649.1604 .

$(R, S, R)-\mathbf{1 1}: \mathbf{m p} 234-238{ }^{\circ} \mathrm{C} ;[\alpha]_{\mathrm{D}^{20}}^{20}=-420\left(\mathrm{CHCl}_{3}, c 0.10\right)$; IR $(\mathrm{KBr}) 3512,3059,2968,2899,1620$, $1508,1439,1425,1389,1329,1111,986,951,827,756 \mathrm{~cm}^{-1} ;{ }^{1} \mathbf{H} \mathbf{~ N M R}\left(400 \mathrm{MHz}, \mathrm{CDCl}_{3}\right) \delta 5.83(\mathrm{AB} \mathrm{q}$, $\Delta v=26.8 \mathrm{~Hz}, J=3.2 \mathrm{~Hz}, 4 \mathrm{H}), 5.91(\mathrm{~s}, 2 \mathrm{H}), 7.20(\mathrm{t}, J=7.6 \mathrm{~Hz}, 2 \mathrm{H}), 7.32(\mathrm{t}, J=7.6 \mathrm{~Hz}, 2 \mathrm{H}), 7.40-7.60(\mathrm{~m}$, 10H), $7.75(\mathrm{~d}, J=8.4 \mathrm{~Hz}, 2 \mathrm{H}), 8.00(\mathrm{~d}, J=8.2 \mathrm{~Hz}, 2 \mathrm{H}), 8.05(\mathrm{~d}, J=8.7 \mathrm{~Hz}, 2 \mathrm{H}) ;{ }^{13} \mathbf{C} \mathbf{N M R}(100 \mathrm{MHz}$, $\left.\mathrm{CDCl}_{3}\right) \delta 103.0,116.2,120.9,124.1,125.4,125.5,126.0,126.2,126.5,127.0,127.17,127.21,127.3,128.6$, 130.8, 131.8, 132.0, 132.3, 140.6, 145.7, 151.4; HR MS (ESI $\left.{ }^{+}\right)$Calcd for $\mathrm{C}_{42} \mathrm{H}_{26} \mathrm{O}_{6} \mathrm{Na}$ : 649.1622 [M+Na] $]^{+}$. 
Found: 649.1609 .

\section{Synthesis of $(R, R)-5$}

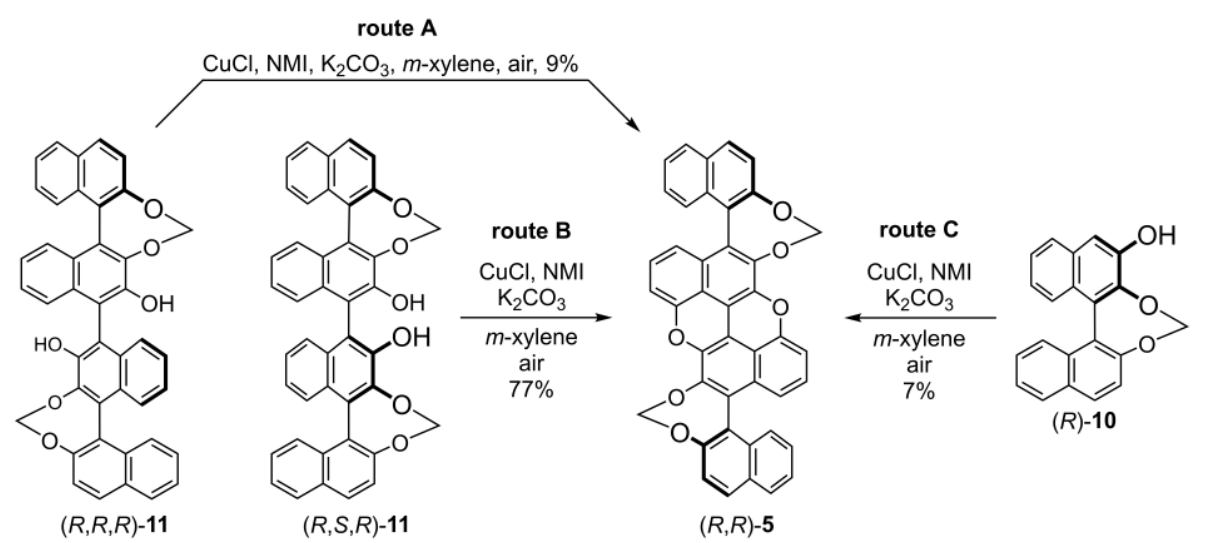

route A; A mixture of $(R, R, R)-\mathbf{1 1}(47.7 \mathrm{mg}, 76.1 \mu \mathrm{mol}), \mathrm{CuCl}(48.7 \mathrm{mg}, 492 \mu \mathrm{mol}), \mathrm{K}_{2} \mathrm{CO}_{3}(51.1 \mathrm{mg}$, $370 \mu \mathrm{mol})$, and $N$-methylimidazole $(25 \mu \mathrm{L}, 0.32 \mathrm{mmol})$ in $m$-xylene $(0.4 \mathrm{~mL})$ was stirred at $120{ }^{\circ} \mathrm{C}$ for $20 \mathrm{~h}$ under air. The mixture was cooled and purified by column chromatography $\left(\mathrm{SiO}_{2}\right.$, hexane/CHCl $\left.3=1 / 2\right)$ to afford $(R, R)-5$ (4.1 mg, $6.6 \mu \mathrm{mol}, 9 \%)$ as an orange powder.

route B; A mixture of $(R, S, R)-\mathbf{1 1}(50.7 \mathrm{mg}, 80.9 \mu \mathrm{mol}), \mathrm{CuCl}(33.9 \mathrm{mg}, 342 \mu \mathrm{mol}), \mathrm{K}_{2} \mathrm{CO}_{3}(38.5 \mathrm{mg}$, $279 \mu \mathrm{mol})$, and $N$-methylimidazole $(25 \mu \mathrm{L}, 0.32 \mathrm{mmol})$ in $m$-xylene $(0.4 \mathrm{~mL})$ was stirred at $120{ }^{\circ} \mathrm{C}$ for $20 \mathrm{~h}$ under air. The mixture was cooled, diluted with water, and extracted with EtOAc (three times). The organic layer was washed with successively water, brine, and $1 \mathrm{M} \mathrm{HCl}$ aq. After dried over $\mathrm{Na}_{2} \mathrm{SO}_{4}$, the solvent was evaporated to give a residue. The residue was purified by column chromatography $\left(\mathrm{SiO}_{2}, \mathrm{CHCl}_{3}\right)$ to afford $(R, R)-5(38.7 \mathrm{mg}, 62.2 \mu \mathrm{mol}, 77 \%)$ as an orange powder.

route C; A mixture of $(R)-\mathbf{1 0}(50.9 \mathrm{mg}, 162 \mu \mathrm{mol}), \mathrm{CuCl}(40.8 \mathrm{mg}, 412 \mu \mathrm{mol}), \mathrm{K}_{2} \mathrm{CO}_{3}(44.4 \mathrm{mg}, 321$ $\mu \mathrm{mol})$, and $N$-methylimidazole $(25 \mu \mathrm{L}, 0.32 \mathrm{mmol})$ in $m$-xylene $(0.8 \mathrm{~mL})$ was stirred at $120{ }^{\circ} \mathrm{C}$ for $20 \mathrm{~h}$ under air. The mixture was cooled, and the solvent was evaporated to give a residue. The residue was purified by column chromatography $\left(\mathrm{SiO}_{2}, \mathrm{CHCl}_{3} /\right.$ hexane $\left.=1 / 1\right)$ to afford $(R, R)-5(3.5 \mathrm{mg}, 5.6 \mu \mathrm{mol}, 7 \%)$ as an orange powder.

$\mathbf{m p}>300{ }^{\circ} \mathrm{C} ;[\alpha]_{\mathrm{D}}{ }^{24}=-337\left(\mathrm{CHCl}_{3}, c 0.10\right) ; \mathbf{I R}(\mathrm{KBr}) 3053,2922,2897,2853,1628,1503,1422,1391$, 1335, 1262, 1206, 1069, 1016, 829, 806, $752 \mathrm{~cm}^{-1}$; ${ }^{1} \mathbf{H}$ NMR $\left(400 \mathrm{MHz}, \mathrm{CDCl}_{3}\right) \delta 5.77$ (AB q, $\Delta v=34.5$ $\mathrm{Hz}, J=3.4 \mathrm{~Hz}, 4 \mathrm{H}), 6.73(\mathrm{~d}, J=8.6 \mathrm{~Hz}, 2 \mathrm{H}), 6.82(\mathrm{~d}, J=7.8 \mathrm{~Hz}, 2 \mathrm{H}), 6.98-7.04(\mathrm{~m}, 2 \mathrm{H}), 7.41$ (ddd $J=$ $1.3,6.9,8.3 \mathrm{~Hz}, 2 \mathrm{H}), 7.46-7.52(\mathrm{~m}, 4 \mathrm{H}), 7.64(\mathrm{~d}, J=8.4 \mathrm{~Hz}, 2 \mathrm{H}), 7.95(\mathrm{~d}, J=8.0 \mathrm{~Hz}, 2 \mathrm{H}), 7.99$ (d, $J=8.7$ $\mathrm{Hz}, 2 \mathrm{H}) ;{ }^{13} \mathrm{C}$ NMR $\left(100 \mathrm{MHz}, \mathrm{CDCl}_{3}\right) \delta 103.2,109.0,113.6,119.2,120.4,121.0,125.4,125.7,126.4$, 126.7, 127.0, 127.5, 128.7, 130.2, 130.8, 131.6, 132.0, 138.3, 141.3, 151.2, 152.5; HR MS (ESI ${ }^{+}$) Calcd for $\mathrm{C}_{42} \mathrm{H}_{22} \mathrm{O}_{6} \mathrm{Na}$ : $645.1309[\mathrm{M}+\mathrm{Na}]^{+}$. Found: 645.1288. 


\section{Determination of the absolute configuration of 7 and 11.}

Each diastereomer of $\mathbf{7}$ and $\mathbf{1 1}$ was converted into $\mathbf{1 2}$ as follows (Scheme S3). X-ray analyses revealed that the absolute configuration of the obtained diastereomer 12 was $R, S, R$ (Figure $\mathrm{S} 1$ ).

Scheme S3. Synthesis of 12 from 7 or 11

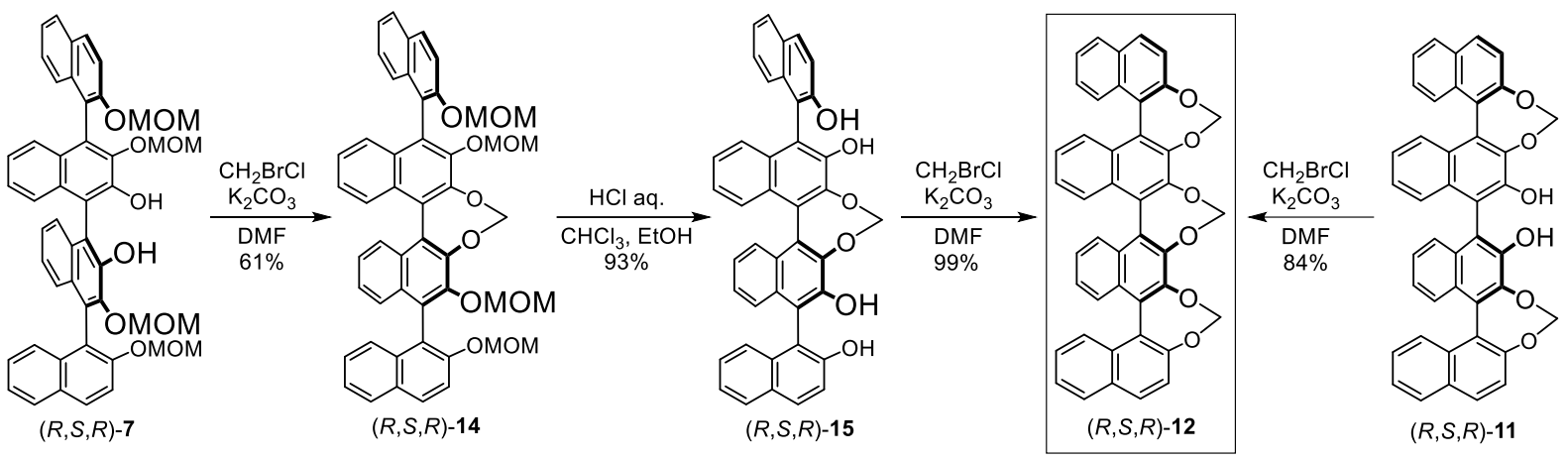

\section{Synthesis of $(R, S, R)-14$}
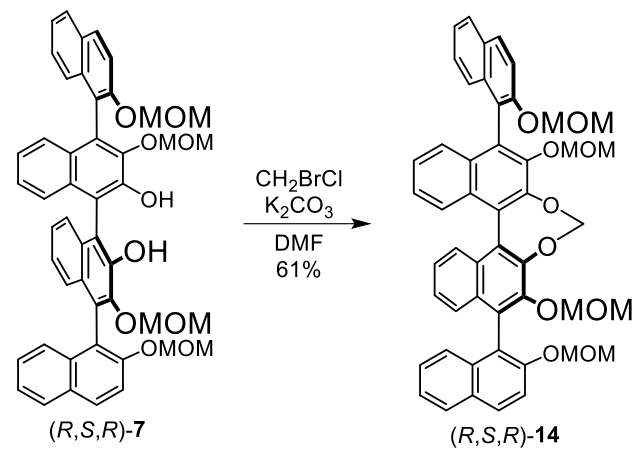

A mixture of $(R, S, R)-7(101 \mathrm{mg}, 130 \mu \mathrm{mol}), \mathrm{CH}_{2} \mathrm{BrCl}(0.25 \mathrm{~mL}, 3.7 \mathrm{mmol})$, and $\mathrm{K}_{2} \mathrm{CO}_{3}(218 \mathrm{mg}, 1.58$ $\mathrm{mmol})$ in dry DMF $(3.7 \mathrm{~mL})$ was stirred at $50{ }^{\circ} \mathrm{C}$ for $13 \mathrm{~h}$ under $\mathrm{N}_{2}$ atmosphere. The reaction mixture was poured into water and EtOAc. The aqueous layer was extracted with EtOAc. The organic layers were combined and washed with water (twice). After dried over $\mathrm{Na}_{2} \mathrm{SO}_{4}$, the solvent was evaporated in vacuo to give a residue. The residue was purified by column chromatography $\left(\mathrm{SiO}_{2}, \mathrm{CHCl}_{3} / \mathrm{EtOAc}=20 / 1\right)$ to afford $(R, S, R)-\mathbf{1 4}(74.2 \mathrm{mg}, 93.8 \mu \mathrm{mol}, 72 \%)$ as a colorless powder.

mp $149-152{ }^{\circ} \mathrm{C} ;[\alpha]_{\mathrm{D}}{ }^{19}=+167\left(\mathrm{CHCl}_{3}, c 0.11\right)$; IR (KBr) 3455, 3063, 2957, 2905, 2826, 1734, 1622, 1472, 1418, 1381, 1260, 1242, 1153, 1051, 1013, 970, 924, 810, $760 \mathrm{~cm}^{-1} ;{ }^{\mathbf{1}} \mathbf{H} \mathbf{N M R}\left(400 \mathrm{MHz}, \mathrm{CDCl}_{3}\right) \delta$ $2.56(\mathrm{~s}, 6 \mathrm{H}), 3.19(\mathrm{~s}, 6 \mathrm{H}), 4.93(\mathrm{AB} \mathrm{q}, \Delta v=37.9 \mathrm{~Hz}, J=6.0 \mathrm{~Hz}, 4 \mathrm{H}), 5.17(\mathrm{AB} \mathrm{q}, \Delta v=56.4 \mathrm{~Hz}, J=6.8 \mathrm{~Hz}$, $4 \mathrm{H}), 5.84(\mathrm{~s}, 2 \mathrm{H}), 7.20-7.35(\mathrm{~m}, 6 \mathrm{H}), 7.35-7.45(\mathrm{~m}, 6 \mathrm{H}), 7.63(\mathrm{~d}, J=9.2 \mathrm{~Hz}, 2 \mathrm{H}), 7.74(\mathrm{~d}, J=8.0 \mathrm{~Hz}, 2 \mathrm{H})$, $7.91(\mathrm{~d}, J=8.4 \mathrm{~Hz}, 2 \mathrm{H}), 8.01(\mathrm{~d}, J=8.8 \mathrm{~Hz}, 2 \mathrm{H}) ;{ }^{13} \mathbf{C} \mathbf{~ N M R}\left(100 \mathrm{MHz}, \mathrm{CDCl}_{3}\right) \delta 56.0,56.1,95.3,98.2$, $102.8,117.2$, 120.9, 124.4, 125.2, 125.7, 126.0, 126.68, 126.73, 127.3, 127.9, 128.2, 128.4, 129.5, 129.9, 130.0, 131.9, 134.3, 145.7, 146.9, 153.1; HR MS (ESI ${ }^{+}$) Calcd for $\mathrm{C}_{49} \mathrm{H}_{42} \mathrm{O}_{10} \mathrm{Na}: 813.2670[\mathrm{M}+\mathrm{Na}]^{+}$. Found: 813.2677. 


\section{Synthesis of $(R, S, R)-15$}
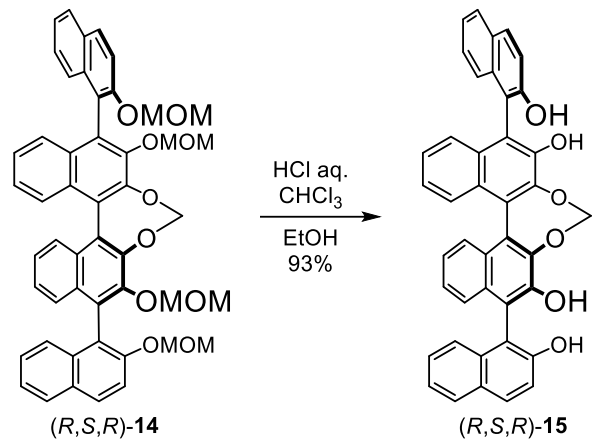

A solution of $(R, S, R)-\mathbf{1 4}(132 \mathrm{mg}, 167 \mu \mathrm{mol})$ in a mixed solvent of $6 \mathrm{M} \mathrm{HCl}$ aq. $(1.7 \mathrm{~mL}), \mathrm{CHCl}_{3}(17$ $\mathrm{mL}$ ), and $\mathrm{EtOH}(3.3 \mathrm{~mL})$ was stirred at $50{ }^{\circ} \mathrm{C}$ for $17 \mathrm{~h}$ under $\mathrm{N}_{2}$ atmosphere. Then water and $\mathrm{CHCl}_{3}$ was added. The organic layer was washed with water (twice). After dried over $\mathrm{Na}_{2} \mathrm{SO}_{4}$, the solvent was evaporated to afford $(R, S, R)-\mathbf{1 5}(95.9 \mathrm{mg}, 156 \mu \mathrm{mol}, 93 \%)$ as a colorless powder.

mp 251-254 ${ }^{\circ} \mathrm{C} ;[\alpha]_{\mathrm{D}^{24}}=+109\left(\mathrm{CHCl}_{3}, c\right.$ 0.10); IR (KBr) 3458, 3061, 2918, 1622, 1508, 1418, 1346, 1267, 1238, 1141, 1090, 1013, 930, 814, $748 \mathrm{~cm}^{-1} ;{ }^{1} \mathbf{H}$ NMR (400 MHz, $\left.\mathrm{CDCl}_{3}\right) \delta 5.21(\mathrm{~s}, 2 \mathrm{H}), 5.83(\mathrm{~s}$, 2H), $5.91(\mathrm{~s}, 2 \mathrm{H}), 7.25-7.45(\mathrm{~m}, 12 \mathrm{H}), 7.45(\mathrm{~d}, J=8.8 \mathrm{~Hz}, 2 \mathrm{H}), 7.74(\mathrm{~d}, J=7.6 \mathrm{~Hz}, 2 \mathrm{H}), 7.92-7.97(\mathrm{~m}$, 2H), $8.02(\mathrm{~d}, J=9.0 \mathrm{~Hz}, 2 \mathrm{H}) ;{ }^{13} \mathbf{C}$ NMR $\left(100 \mathrm{MHz}, \mathrm{CDCl}_{3}\right) \delta 103.1,112.5,114.9,117.9,124.0,124.7$, $124.8,125.2$, 127.1, 127.3, 127.5, 127.6, 127.9, 128.6, 129.5, 131.2, 131.8, 133.6, 140.9, 146.1, 152.2; HR MS $\left(\mathrm{ESI}^{+}\right)$Calcd for $\mathrm{C}_{41} \mathrm{H}_{26} \mathrm{O}_{6} \mathrm{Na}: 637.1622[\mathrm{M}+\mathrm{Na}]^{+}$. Found: 637.1607.

\section{Synthesis of $(R, S, R)-12$}
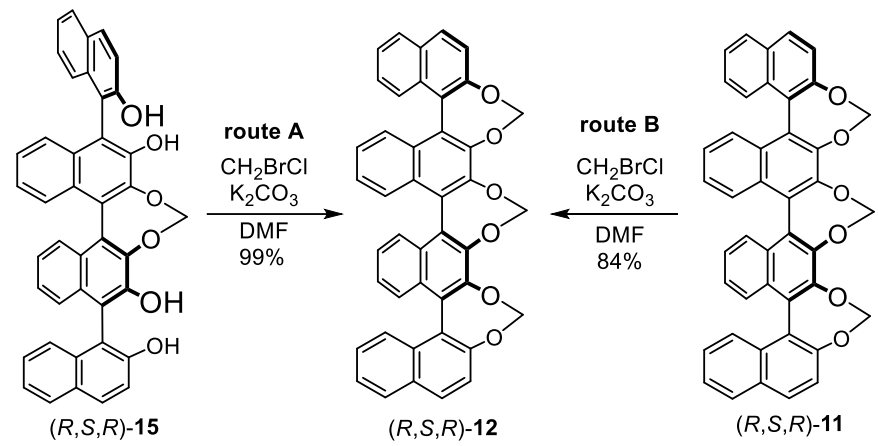

route $\mathrm{A}$; A mixture of $(R, S, R)-\mathbf{1 5}(627 \mathrm{mg}, 1.00 \mathrm{mmol}), \mathrm{CH}_{2} \mathrm{BrCl}(2.01 \mathrm{~mL}, 30.0 \mathrm{mmol})$, and $\mathrm{K}_{2} \mathrm{CO}_{3}$ $(1.38 \mathrm{~g}, 10.0 \mathrm{mmol})$ in dry DMF $(10 \mathrm{~mL})$ was stirred at $50{ }^{\circ} \mathrm{C}$ for $20 \mathrm{~h}$ under $\mathrm{N}_{2}$ atmosphere. The reaction mixture was poured into water. The aqueous layer was extracted with $\mathrm{CHCl}_{3}$. The organic layers were combined and washed successively with water and brine. After dried over $\mathrm{Na}_{2} \mathrm{SO}_{4}$, the solvent was evaporated in vacuo to give a residue. The residue was purified by column chromatography $\left(\mathrm{SiO}_{2}, \mathrm{CHCl}_{3}\right)$ to afford $(R, S, R)-\mathbf{1 2}(634 \mathrm{mg}, 993 \mu \mathrm{mol}, 99 \%)$ as a yellow powder.

route B; A mixture of $(R, S, R)-\mathbf{1 1}(30.1 \mathrm{mg}, 49.0 \mu \mathrm{mol}), \mathrm{CH}_{2} \mathrm{BrCl}(196 \mu \mathrm{L}, 2.92 \mathrm{mmol})$, and $\mathrm{K}_{2} \mathrm{CO}_{3}(137$ $\mathrm{mg}, 991 \mu \mathrm{mol})$ in dry DMF $(1.6 \mathrm{~mL})$ was stirred at $50{ }^{\circ} \mathrm{C}$ for $15 \mathrm{~h}$ under $\mathrm{N}_{2}$ atmosphere. The reaction 
mixture was poured into water. The aqueous layer was extracted with EtOAc (twice). The organic layers were combined and washed with water (twice). After dried over $\mathrm{Na}_{2} \mathrm{SO}_{4}$, the solvent was evaporated in vacuo to give a residue. The residue was purified by column chromatography $\left(\mathrm{SiO}_{2}, \mathrm{CHCl}_{3}\right)$ to afford $(R, S, R)-\mathbf{1 2}(26.2 \mathrm{mg}, 41.0 \mu \mathrm{mol}, 84 \%)$ as a yellow powder.

$\mathbf{m p}>300{ }^{\circ} \mathrm{C} ;[\alpha]_{\mathrm{D}}{ }^{19}=-315\left(\mathrm{CHCl}_{3}, c 0.10\right) ; \mathbf{I R}(\mathrm{KBr}) 3057,2965,2901,1620,1508,1485,1421,1383$, 1329, 1263, 1215, 1140, 1026, 988, 941, 908, 827, $804 \mathrm{~cm}^{-1} ;{ }^{1} \mathbf{H}$ NMR (400 MHz, $\left.\mathrm{CDCl}_{3}\right) \delta 5.86(\mathrm{AB} \mathrm{q}$, $\Delta v=22.4 \mathrm{~Hz}, J=3.4 \mathrm{~Hz}, 4 \mathrm{H}), 5.93(\mathrm{~s}, 2 \mathrm{H}), 7.30(\mathrm{t}, J=7.6 \mathrm{~Hz}, 2 \mathrm{H}), 7.37(\mathrm{t}, J=7.0 \mathrm{~Hz}, 2 \mathrm{H}), 7.45(\mathrm{t}, J=$ $7.1 \mathrm{~Hz}, 2 \mathrm{H}), 7.52(\mathrm{~d}, J=7.1 \mathrm{~Hz}, 2 \mathrm{H}), 7.56(\mathrm{~d}, J=8.7 \mathrm{~Hz}, 2 \mathrm{H}), 7.62(\mathrm{~d}, J=8.3 \mathrm{~Hz}, 2 \mathrm{H}), 7.76(\mathrm{~d}, J=8.4 \mathrm{~Hz}$, 2H), $7.80(\mathrm{~d}, J=8.0 \mathrm{~Hz}, 2 \mathrm{H}), 8.00(\mathrm{~d}, J=8.0 \mathrm{~Hz}, 2 \mathrm{H}), 8.06(\mathrm{~d}, J=8.7 \mathrm{~Hz}, 2 \mathrm{H}) ;{ }^{13} \mathbf{C} \mathbf{N M R}(100 \mathrm{MHz}$, $\left.\mathrm{CDCl}_{3}\right) \delta 103.9,104.2,121.1,125.4,125.66,125.74,126.1,126.6,127.1,127.6,127.7,128.5,128.7,129.0$, 130.3, 130.4, 131.0, 132.1, 132.3, 144.7, 144.9, 151.6; HR MS (ESI ${ }^{+}$Calcd for $\mathrm{C}_{43} \mathrm{H}_{26} \mathrm{O}_{6} \mathrm{Na}$ : 661.1622 $[\mathrm{M}+\mathrm{Na}]^{+}$. Found: 661.1614 . 


\section{X-ray structures}

Single crystals of $(R, R)-\mathbf{5}$ and $(R, S, R)-\mathbf{1 2}$ were obtained by vapor diffusion of $\mathrm{CHCl}_{3} / \mathrm{EtOH}$ and $\mathrm{CHCl}_{3}$ solution, respectively. Data of $(R, R)-\mathbf{5}$ were collected by a Rigaku Varimax with RAPID diffractometer using multi-layer mirror monochromated $\mathrm{Cu}-\mathrm{K} \alpha$ radiation. Data of $(R, S, R)-\mathbf{1 2}$ were collected by a Rigaku Saturn724 diffractometer using multi-layer mirror monochromated Mo-K $\alpha$ radiation. CIF files CCDC 1918068 for $(R, R)-\mathbf{5}$ and 1909712 for $(R, S, R)$-12 can be obtained free of charge from the Cambridge Crystallographic Data Centre via www.ccdc.cam.ac.uk/data_request/cif.
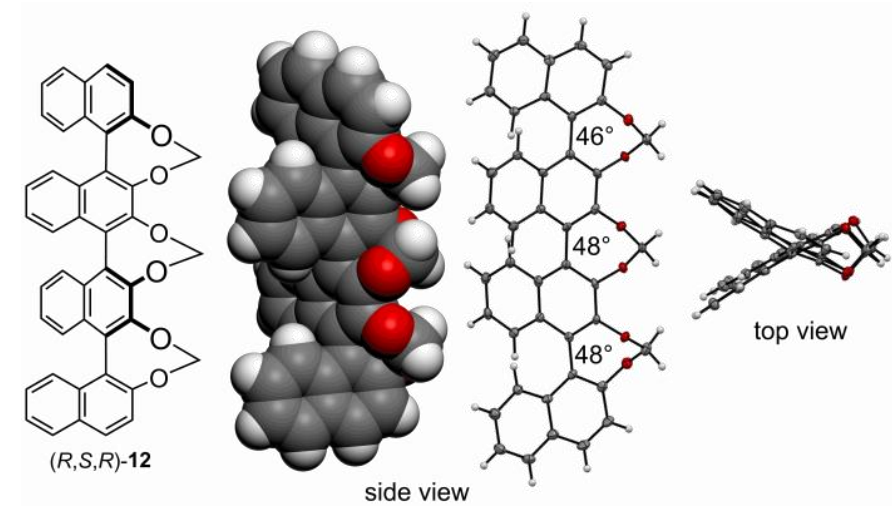

Figure S1. X-ray crystal structure of $(R, S, R)$-12. The solvent molecules are omitted for clarity. Thermal ellipsoids are set at $50 \%$ probability.

(a)

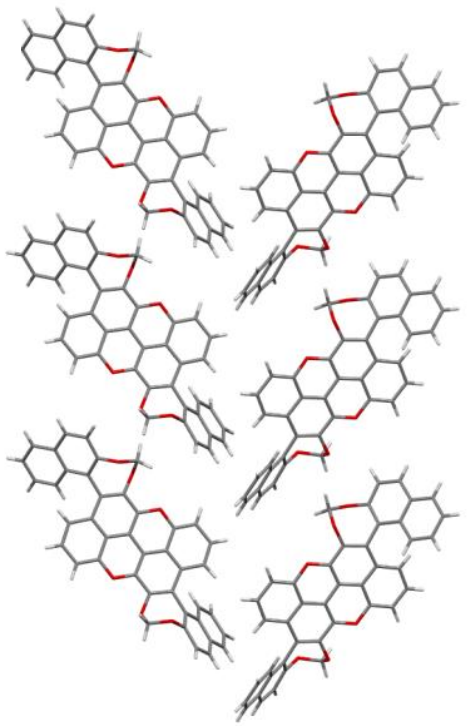

(b)

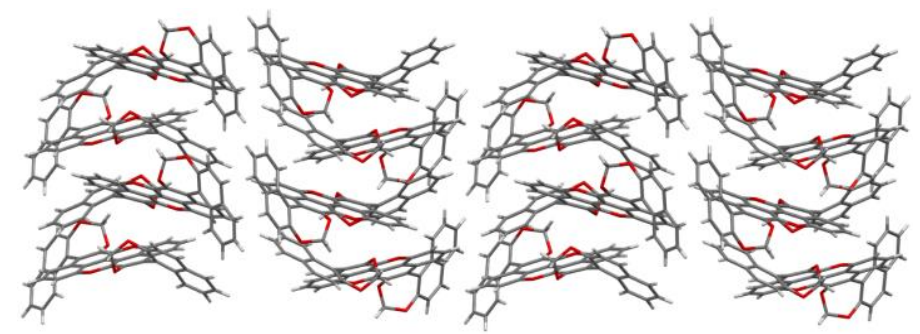

Figure S2. (a) One-layer-top view and (b) side view of crystal packing of $(R, R)-\mathbf{5}$. 


\section{DFT calculations}

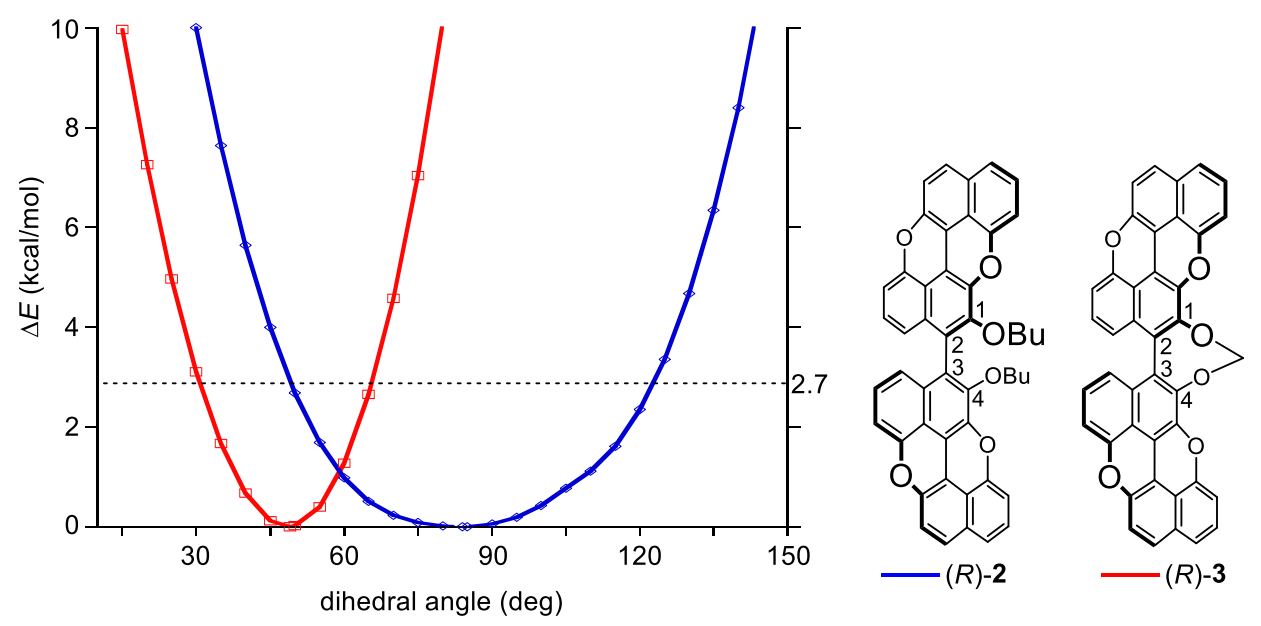

Figure S3. Relationship between the energy difference and biaryl dihedral angle of $\mathrm{C}(1)-\mathrm{C}(2)-\mathrm{C}(3)-\mathrm{C}(4)$ of $(R)-2$ and $(R)-3$. DFT calculations were performed at the B3LYP/6-31G(d,p) level.

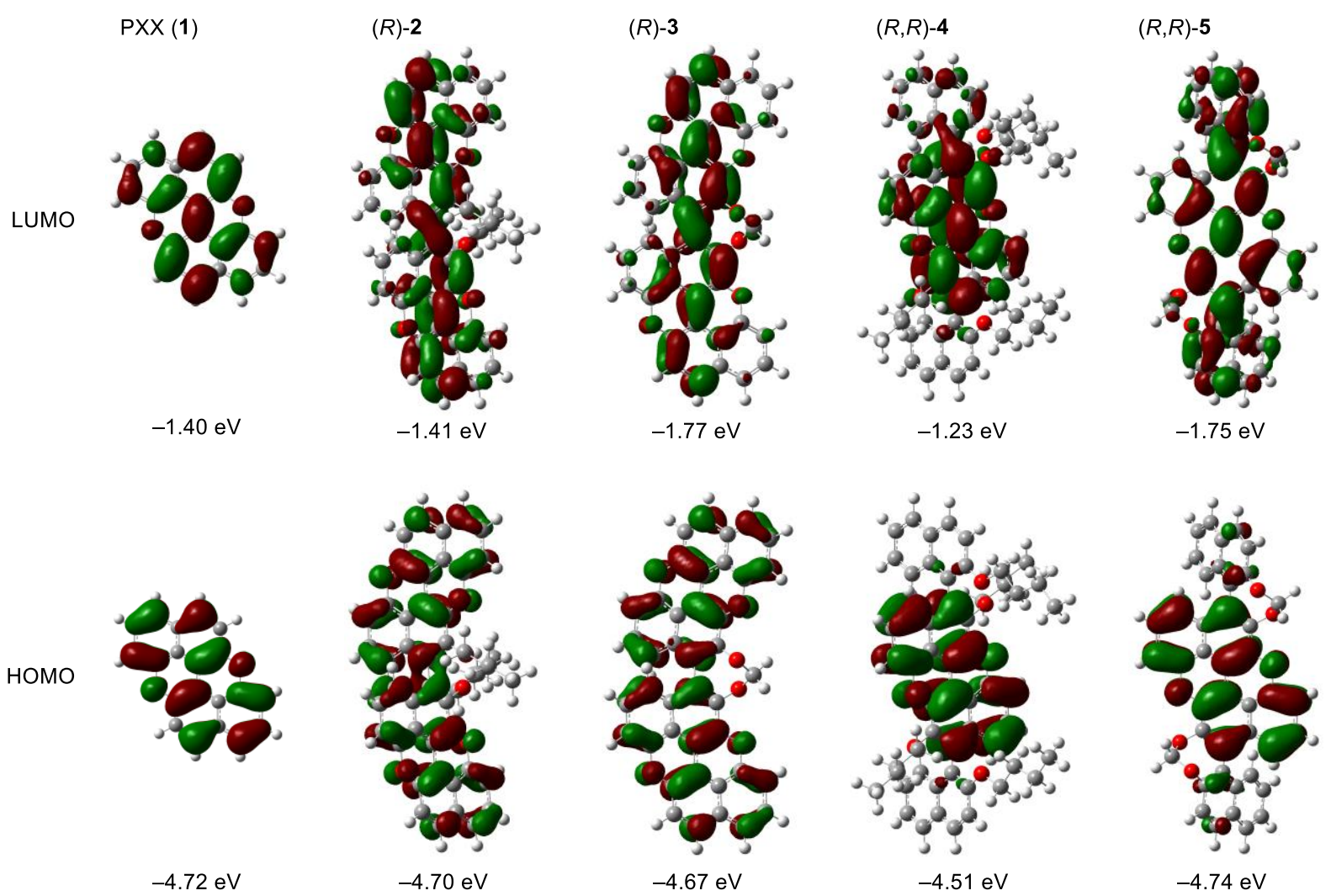

Figure S4. Calculated HOMOs and LUMOs of 1-5. DFT calculations were performed at the B3LYP/6-31G(d,p) level. 


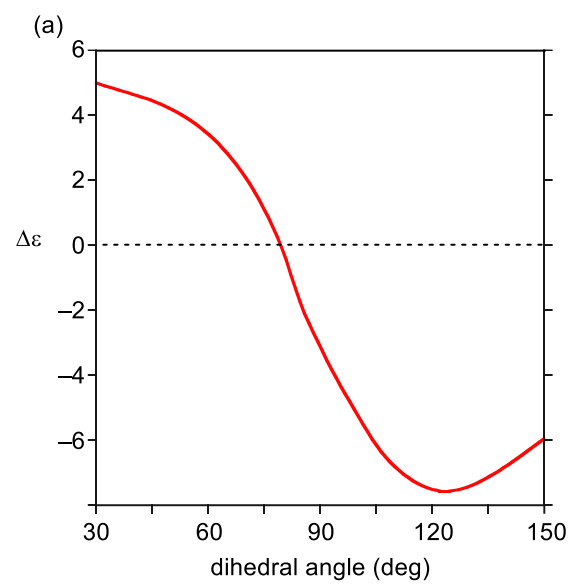

(b)

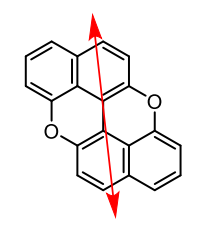

Figure S5. (a) Relationship between the CD intensity at 450-500 nm and dihedral angle of PXX rings of $(R)$-2. TD DFT calculations were performed at the B3LYP/6-31G $(\mathrm{d}, \mathrm{p})$ level. Half-width at half height $=$ $2500 \mathrm{~cm}^{-1}$. (b) A calculated electric dipole moment of PXX (1) at the B3LYP/6-31G(d,p) level.

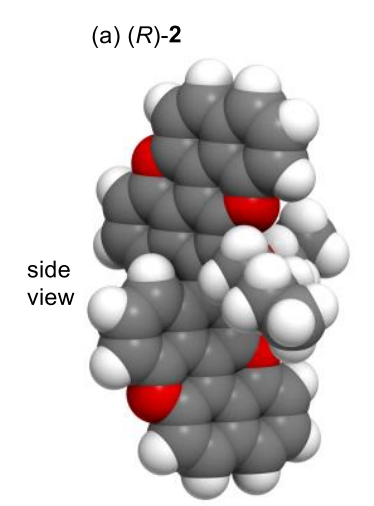

(b) $(R)-3$
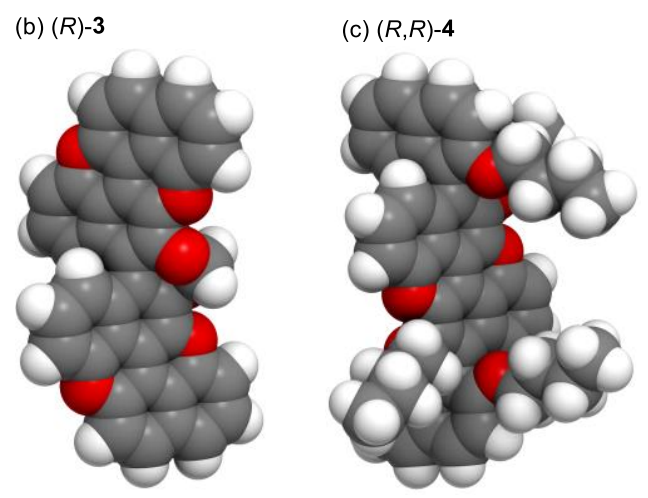

(d) $(R, R)-5$
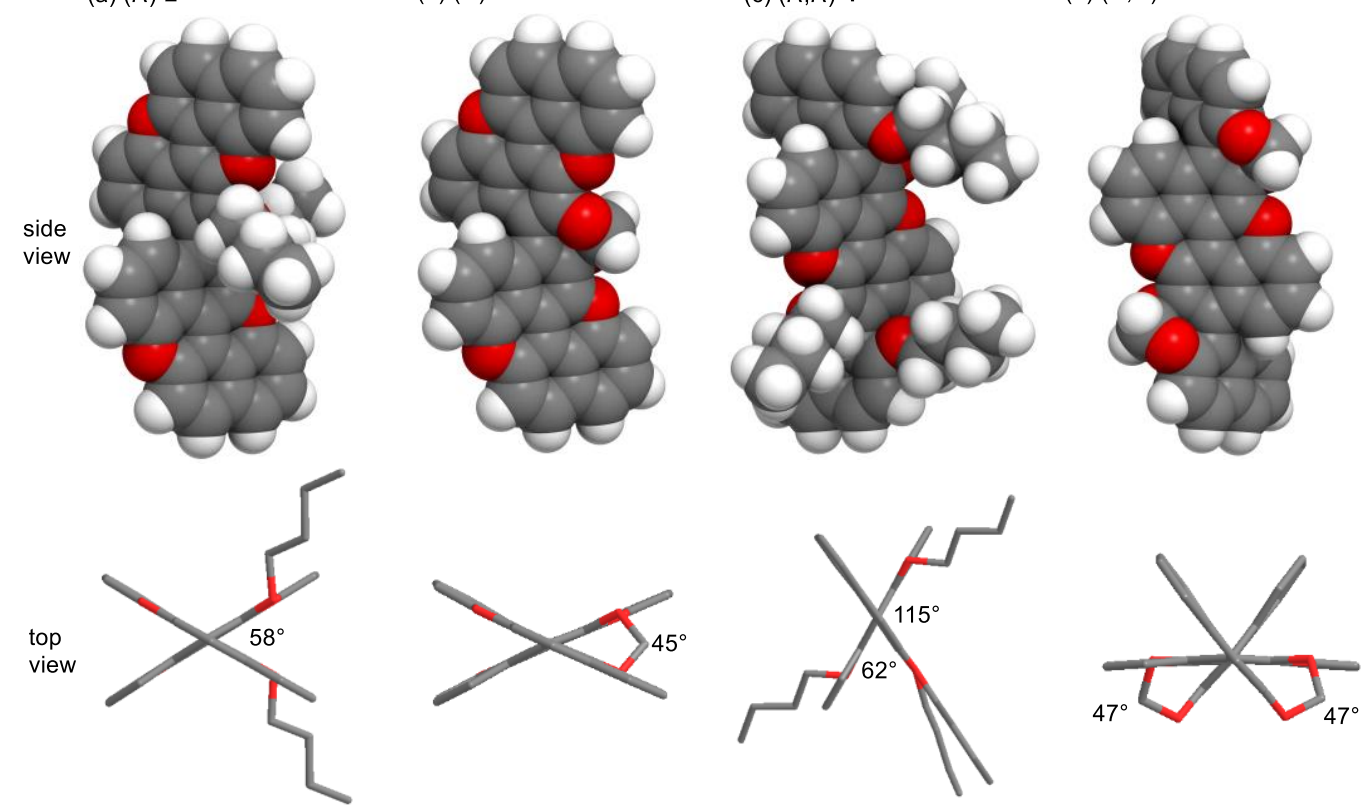

Figure S6. TD DFT-optimized excited states of 2-5 at the B3LYP/6-31G(d,p) level. The optimized ground-state structure was used as an initial structure. 


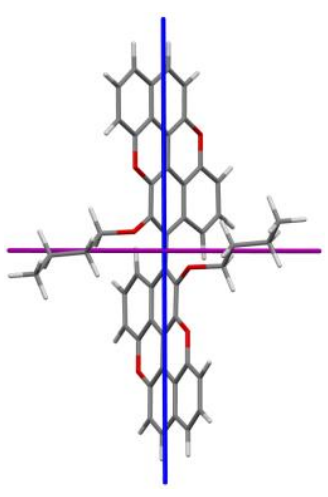

$(R)-2$

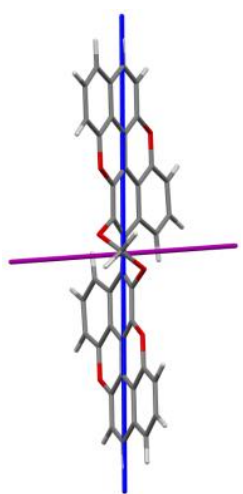

$(R)-3$

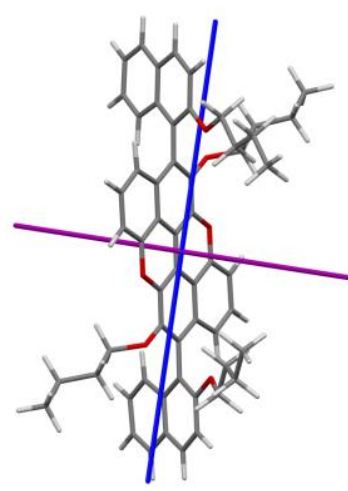

$(R, R)-4$

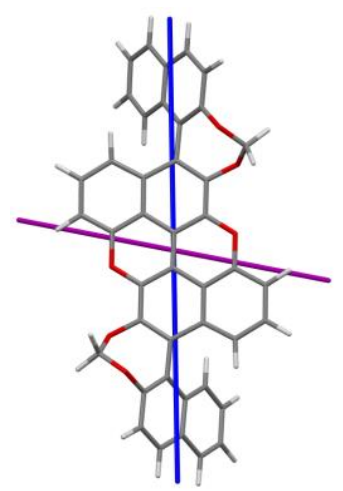

$(R, R)-5$

Figure S7. Orientations of the electric (blue) and magnetic (purple) transition dipole moments of 2-5 at the CAM-B3LYP/6-311+G(2d,p) level with the SCRF method (1,4-dioxane). 


\section{FL and CPL spectra}

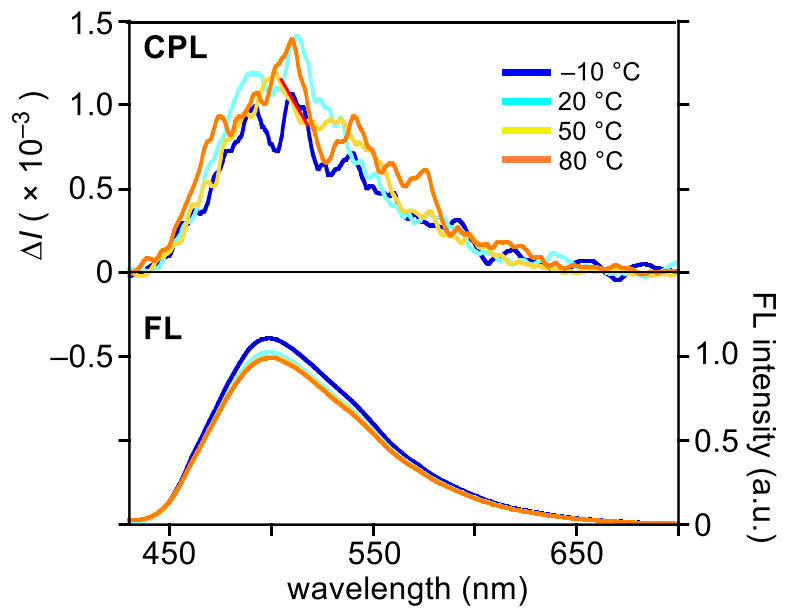

Figure S8. CPL and FL spectra of $(R, R)-5$. Conditions: 1-octanol, $5.0 \times 10^{-6} \mathrm{M},-10$ to $80{ }^{\circ} \mathrm{C}$, light path length $=1 \mathrm{~cm}, \lambda_{\mathrm{ex}}=350 \mathrm{~nm}$.

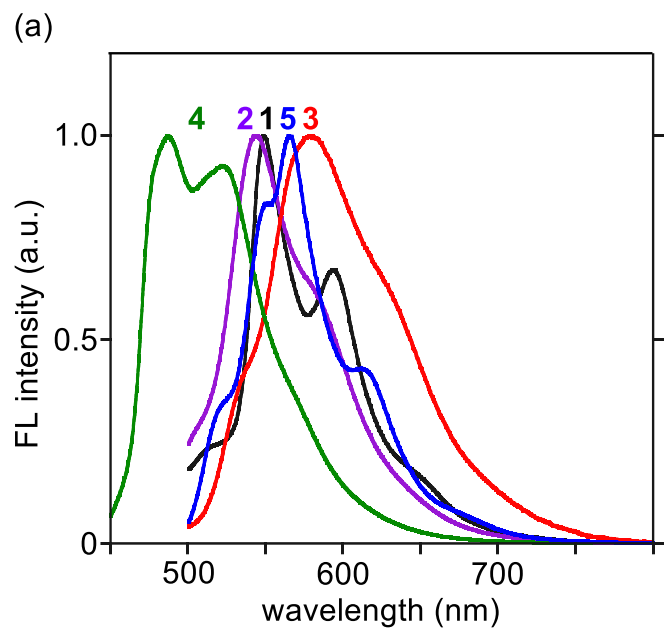

(b)
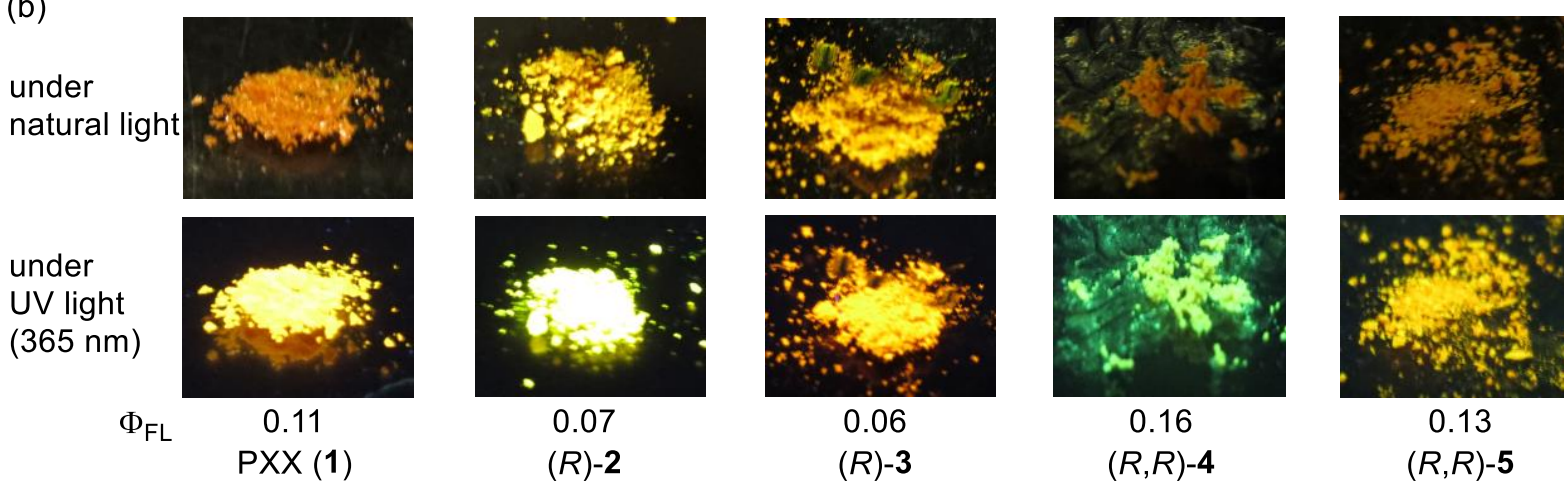

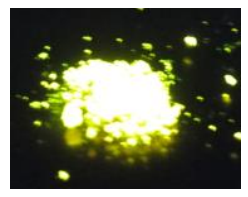

0.07

$(R)-2$

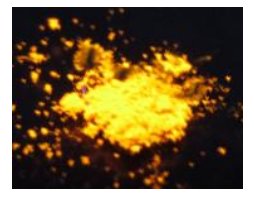

0.06

$(R)-3$

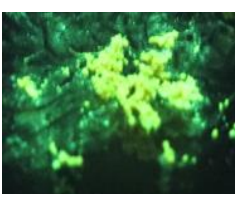

0.16 $(R, R)-4$

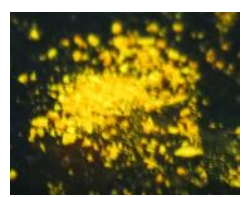

0.13

$(R, R)-5$

Figure S9. (a) FL spectra and (b) photographs of 1-5 in the solid state. $\lambda_{\text {ex }}=400 \mathrm{~nm}$, rt. 

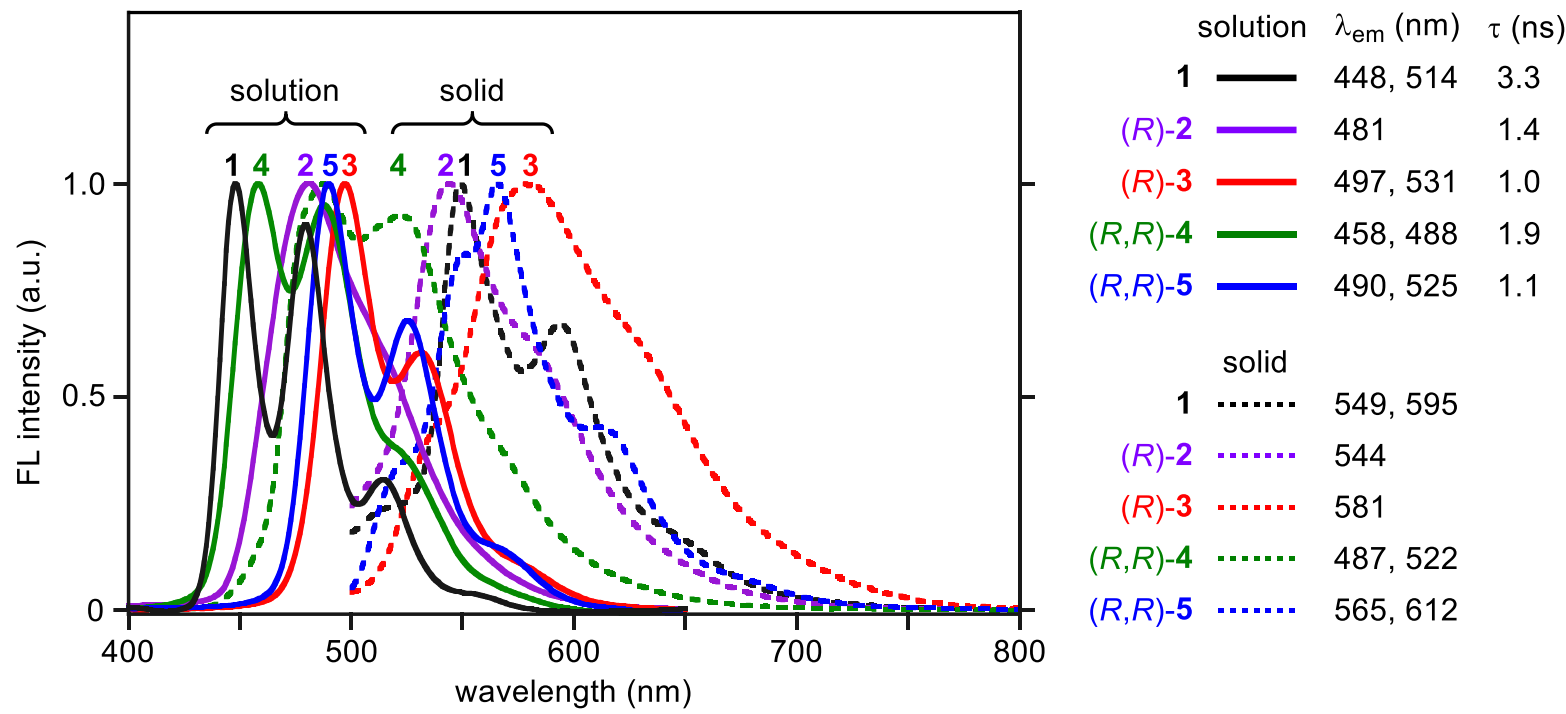

Figure S10. FL spectra of $\mathbf{1 - 5}$ in solution (solid line) and in the solid state (dotted line). Conditions for solutions: 1,4-dioxane, $5.0 \times 10^{-6} \mathrm{M}, 20^{\circ} \mathrm{C}, l=1 \mathrm{~cm}, \lambda_{\mathrm{ex}}=350 \mathrm{~nm}$. Conditions for the solid state: $\lambda_{\mathrm{ex}}=$ $400 \mathrm{~nm}$, rt. 


\section{6. ${ }^{1} \mathrm{H}$ NMR and ${ }^{13} \mathrm{C}$ NMR spectra}
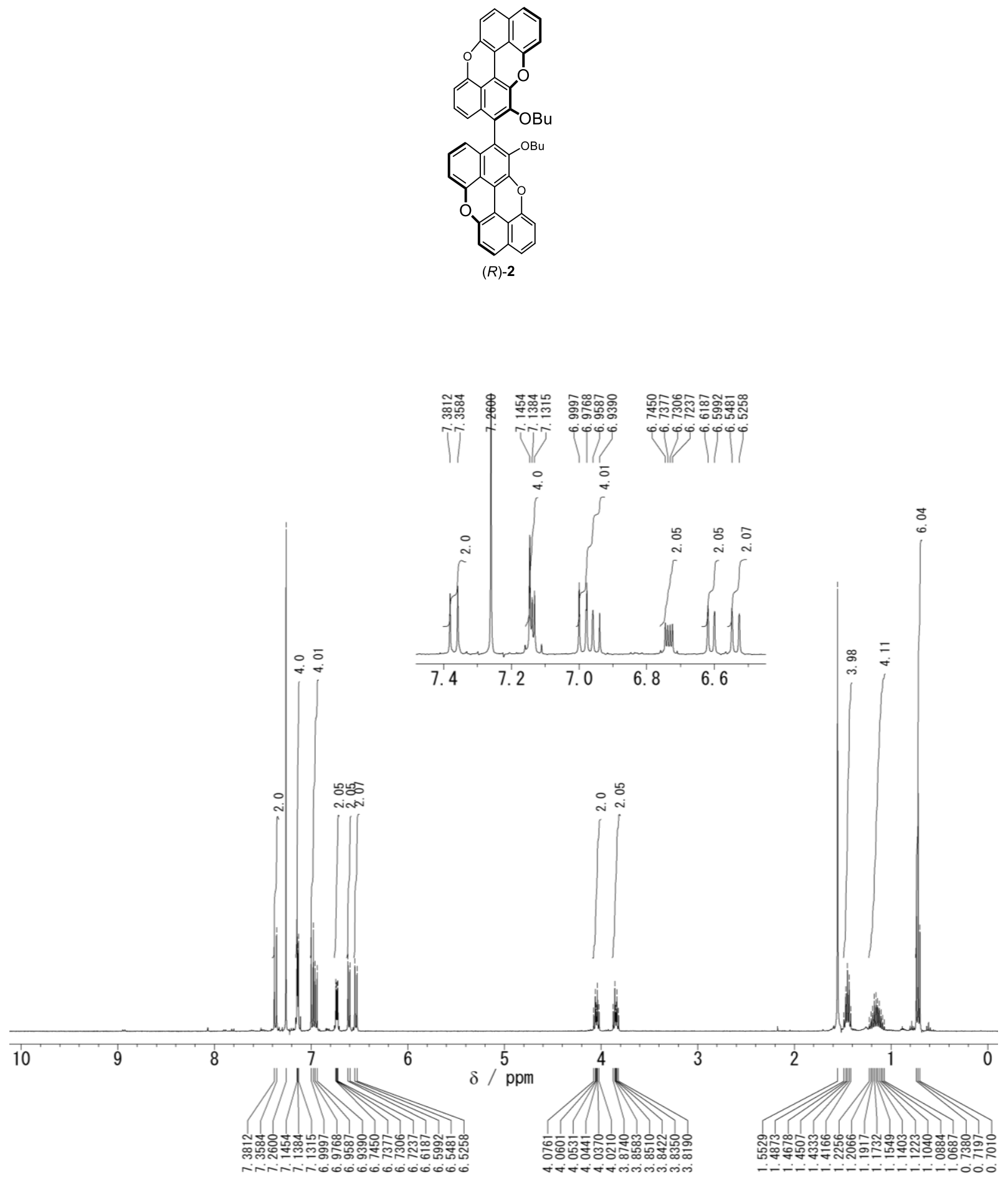

${ }^{1} \mathrm{H}$ NMR of $(R)-2\left(400 \mathrm{MHz}, \mathrm{CDCl}_{3}\right)$ 


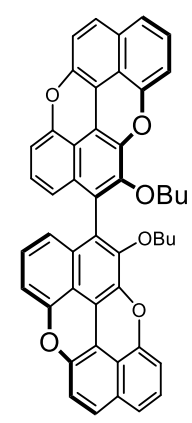

(R)-2

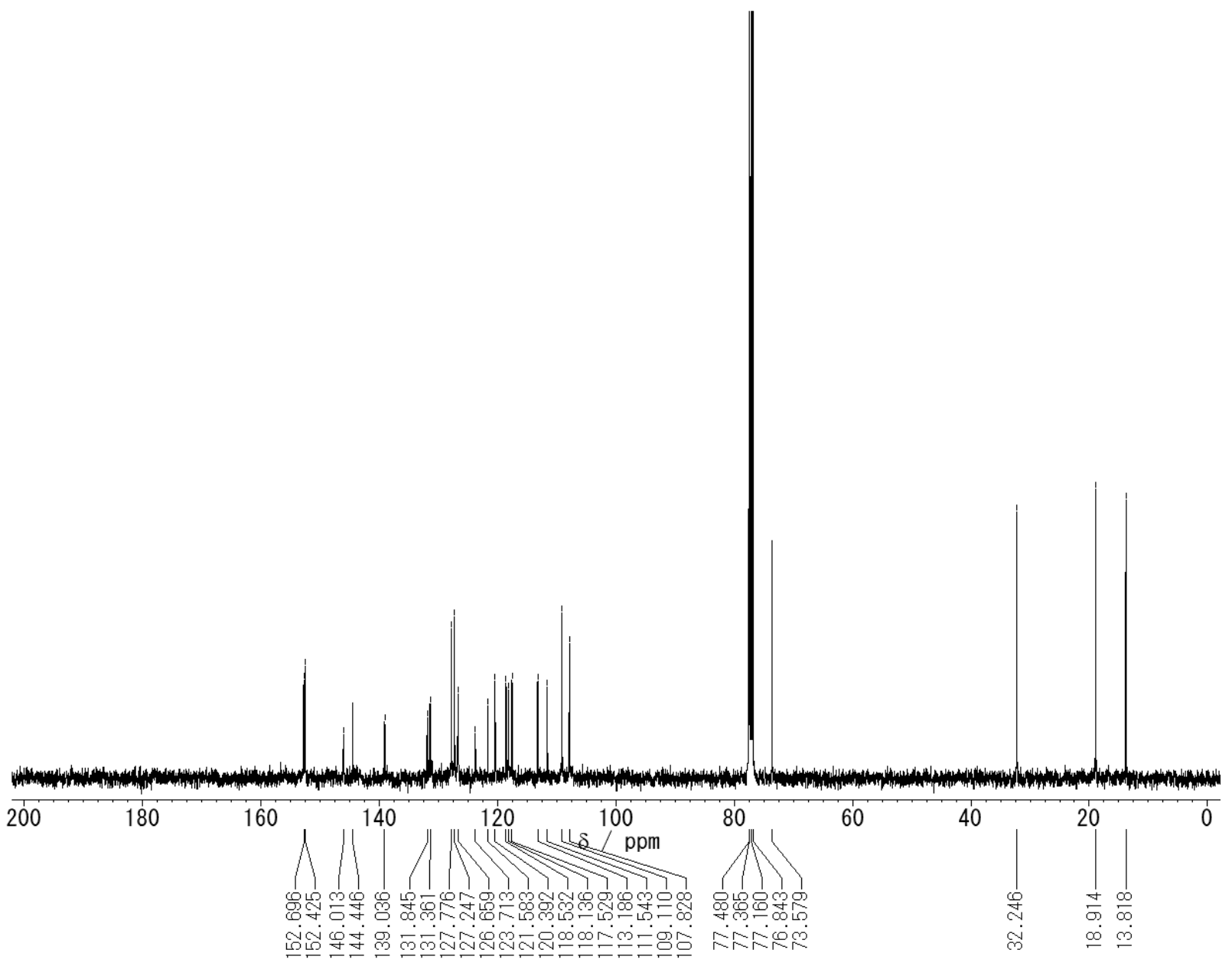

${ }^{13} \mathrm{C}$ NMR of $(R)-2\left(100 \mathrm{MHz}, \mathrm{CDCl}_{3}\right)$ 

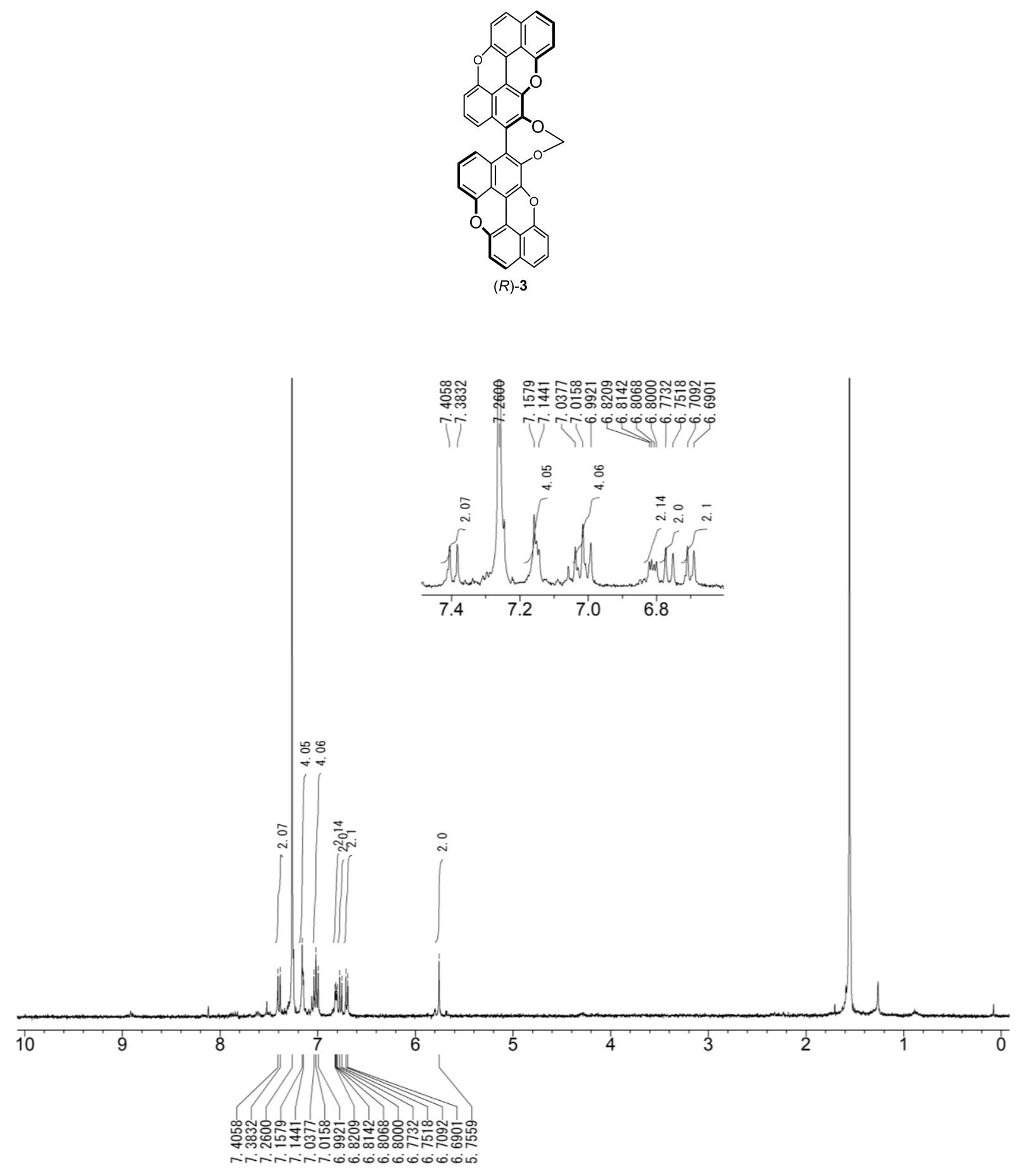

${ }^{1} \mathrm{H}$ NMR of $(R)-3\left(400 \mathrm{MHz}, \mathrm{CDCl}_{3}\right)$ 

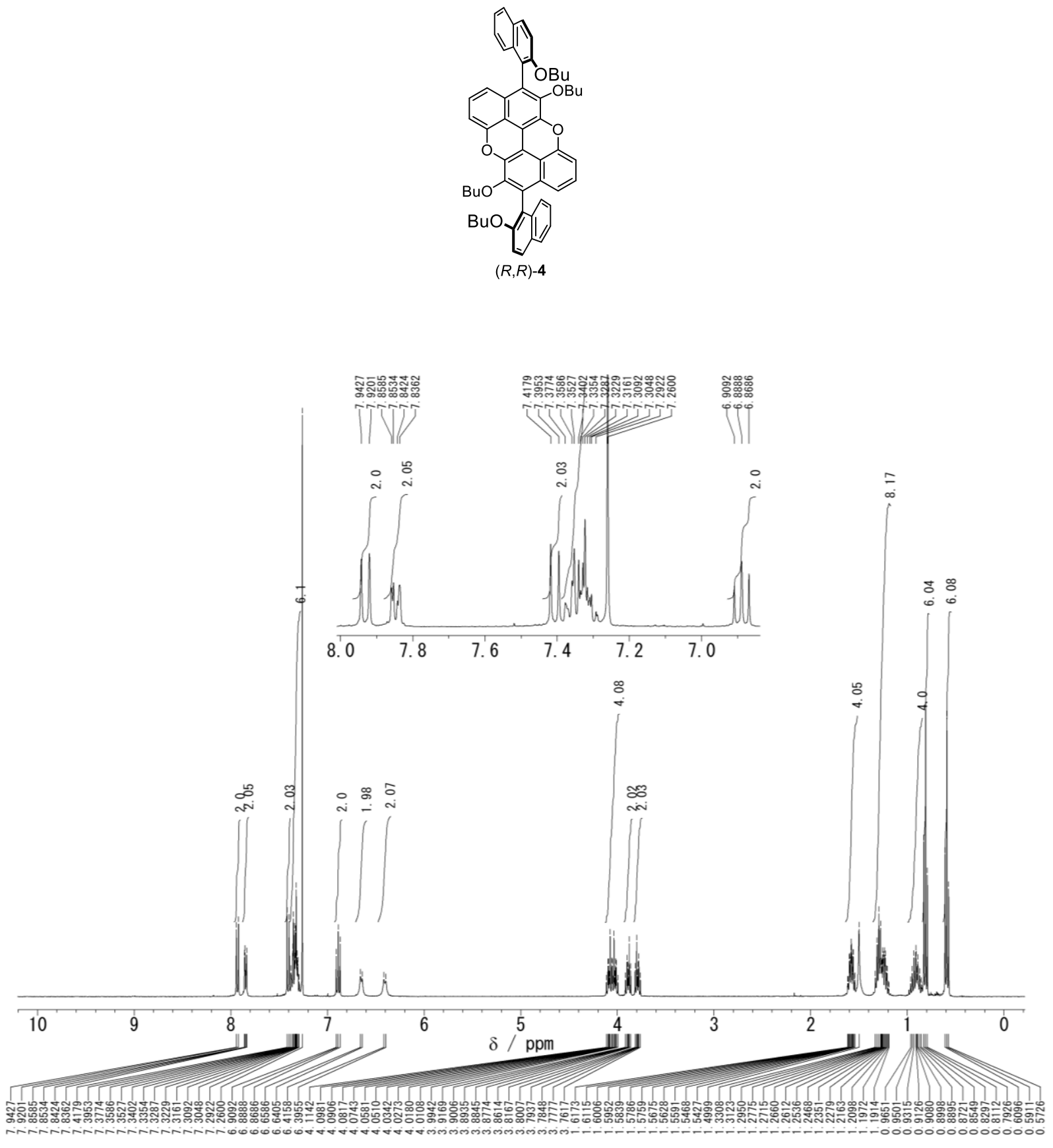

${ }^{1} \mathrm{H} \mathrm{NMR}$ of $(R, R)-\mathbf{4}\left(400 \mathrm{MHz}, \mathrm{CDCl}_{3}\right)$ 

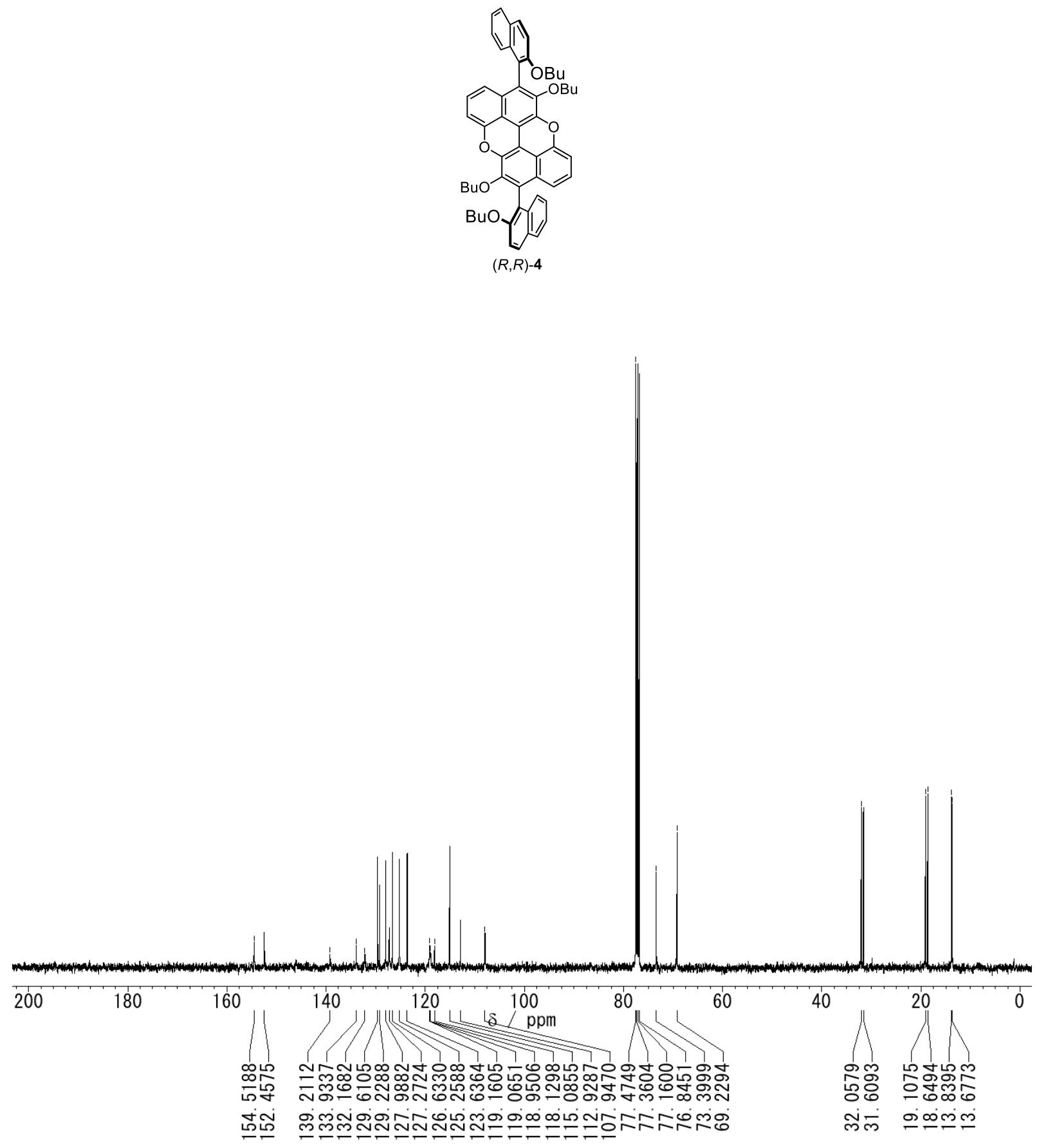

${ }^{13} \mathrm{C}$ NMR of $(R, R)-4\left(100 \mathrm{MHz}, \mathrm{CDCl}_{3}\right)$ 

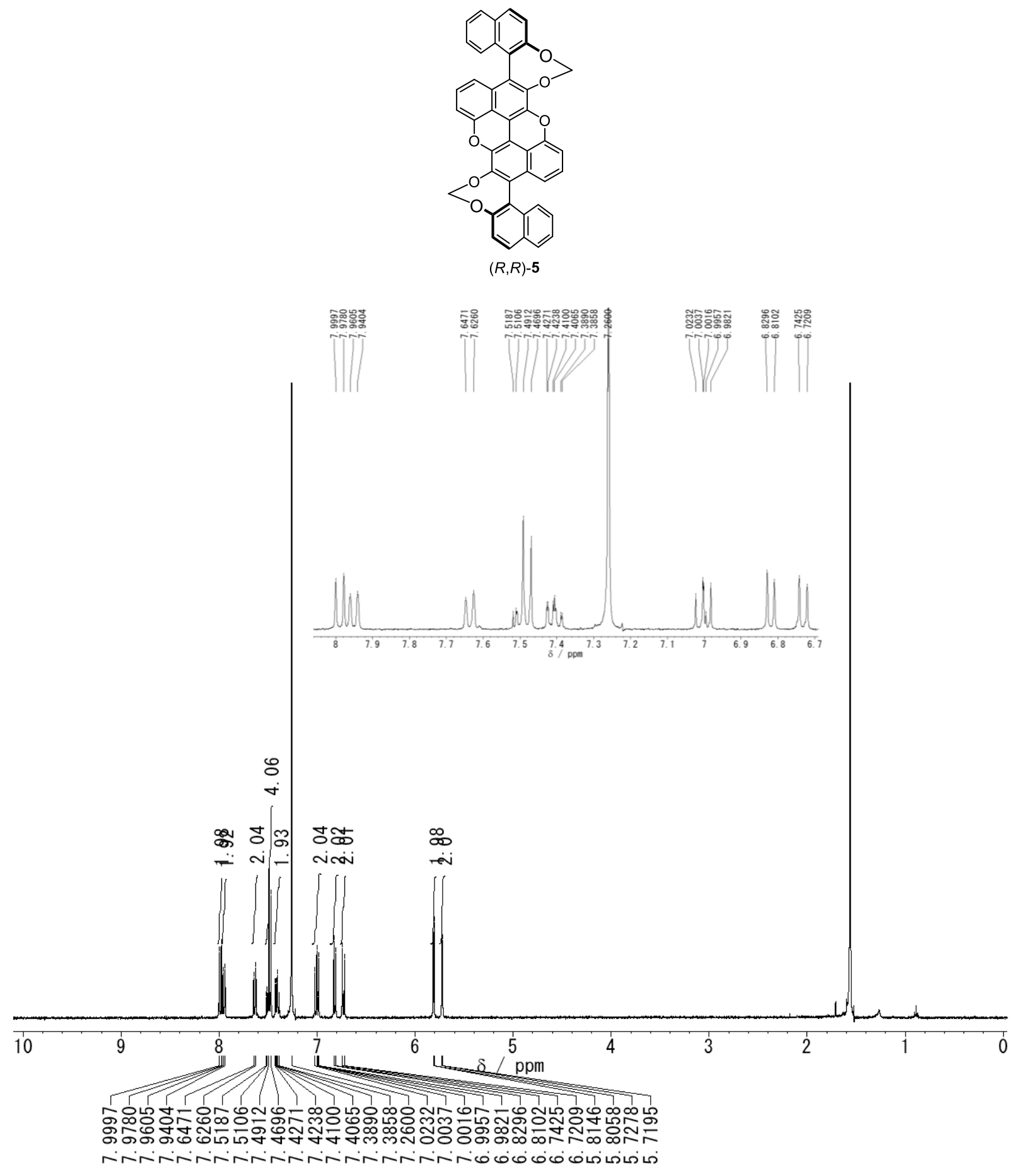

${ }^{1} \mathrm{H}$ NMR of $(R, R)-5\left(400 \mathrm{MHz}, \mathrm{CDCl}_{3}\right)$ 


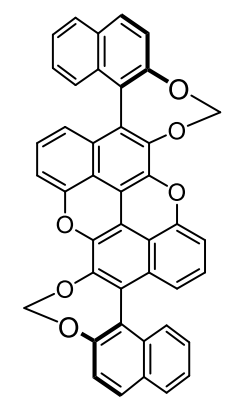

$(R, R)-5$

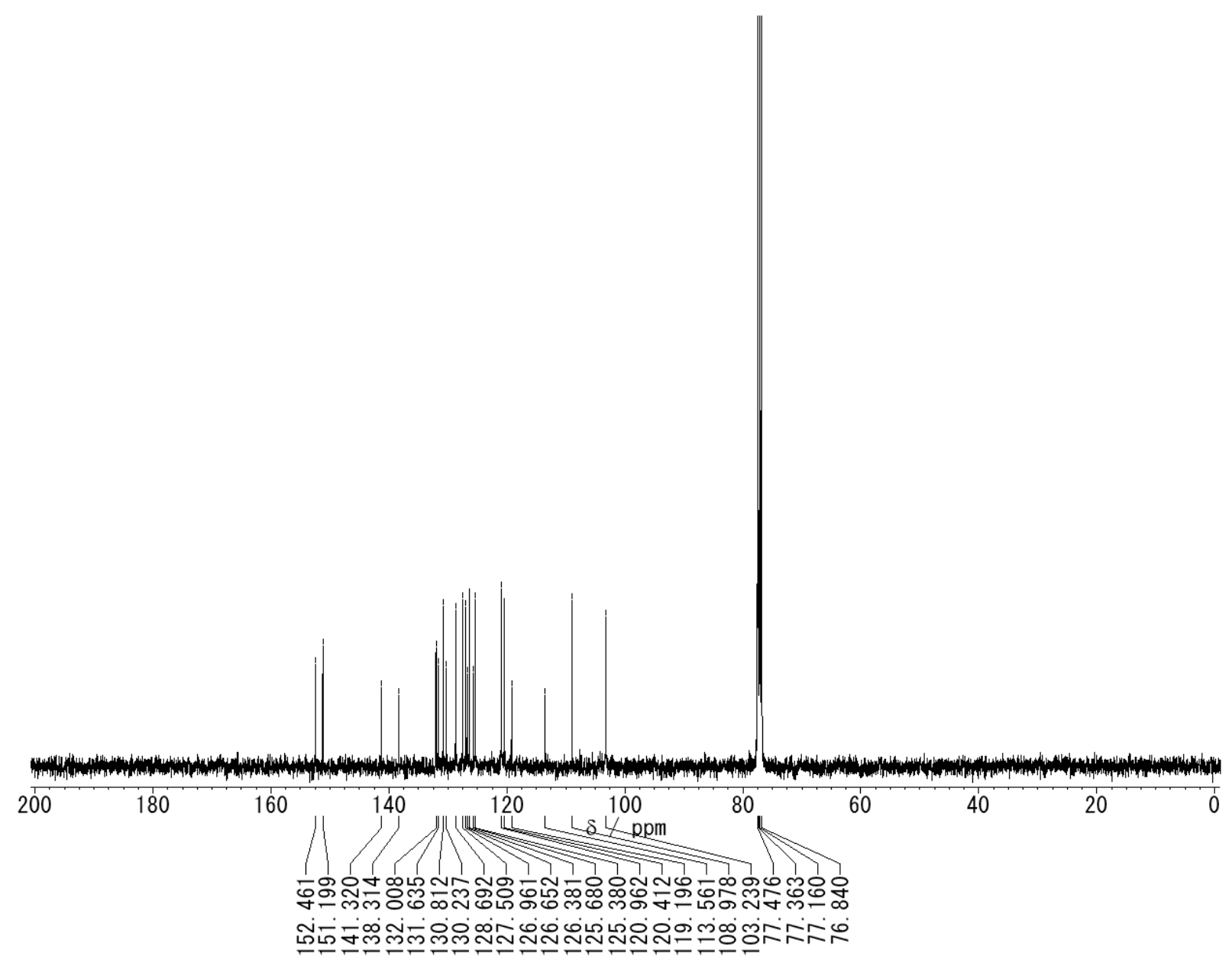

${ }^{13} \mathrm{C}$ NMR of $(R, R)-5\left(100 \mathrm{MHz}, \mathrm{CDCl}_{3}\right)$ 

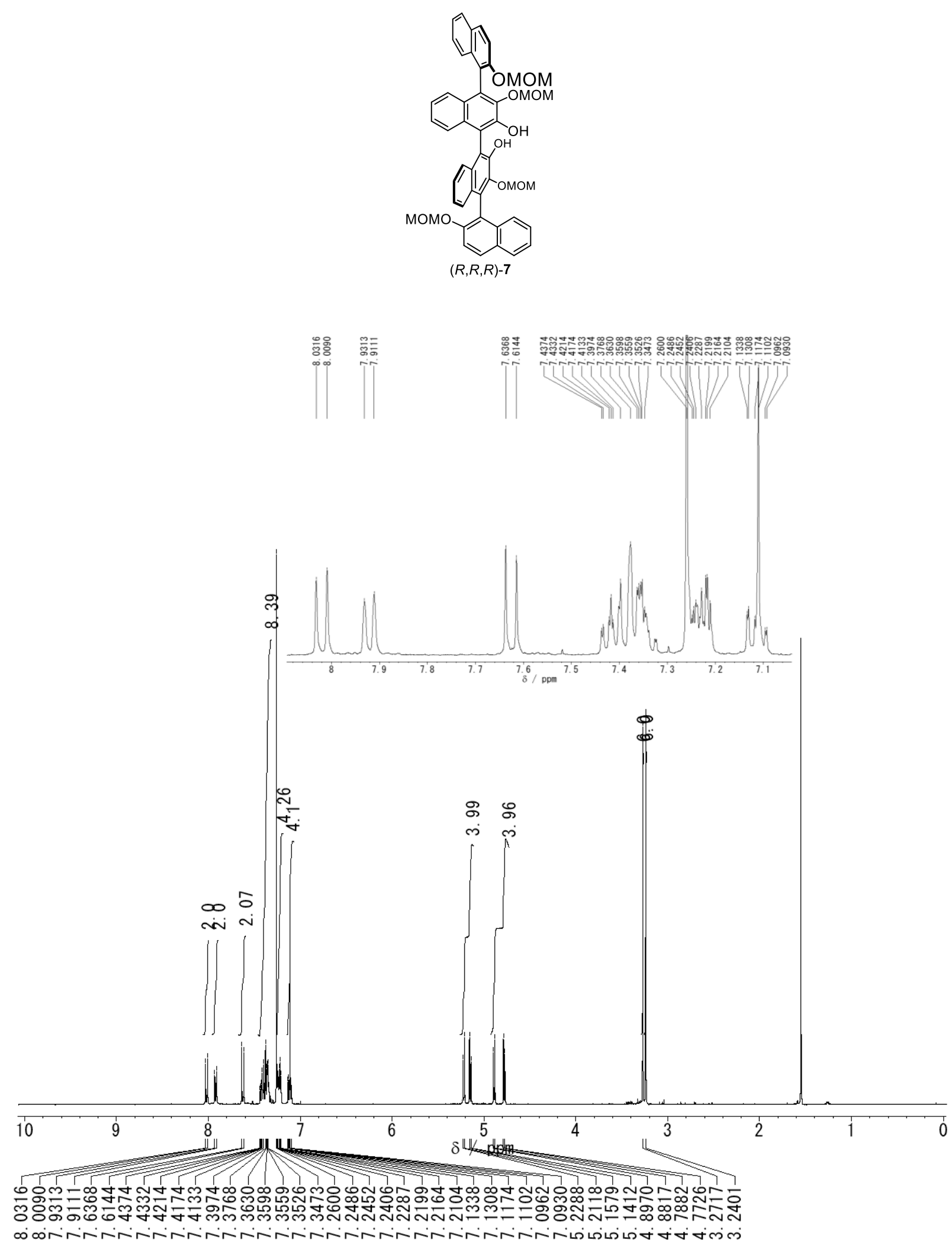

${ }^{1} \mathrm{H}$ NMR of $(R, R, R)-7\left(400 \mathrm{MHz}, \mathrm{CDCl}_{3}\right)$ 

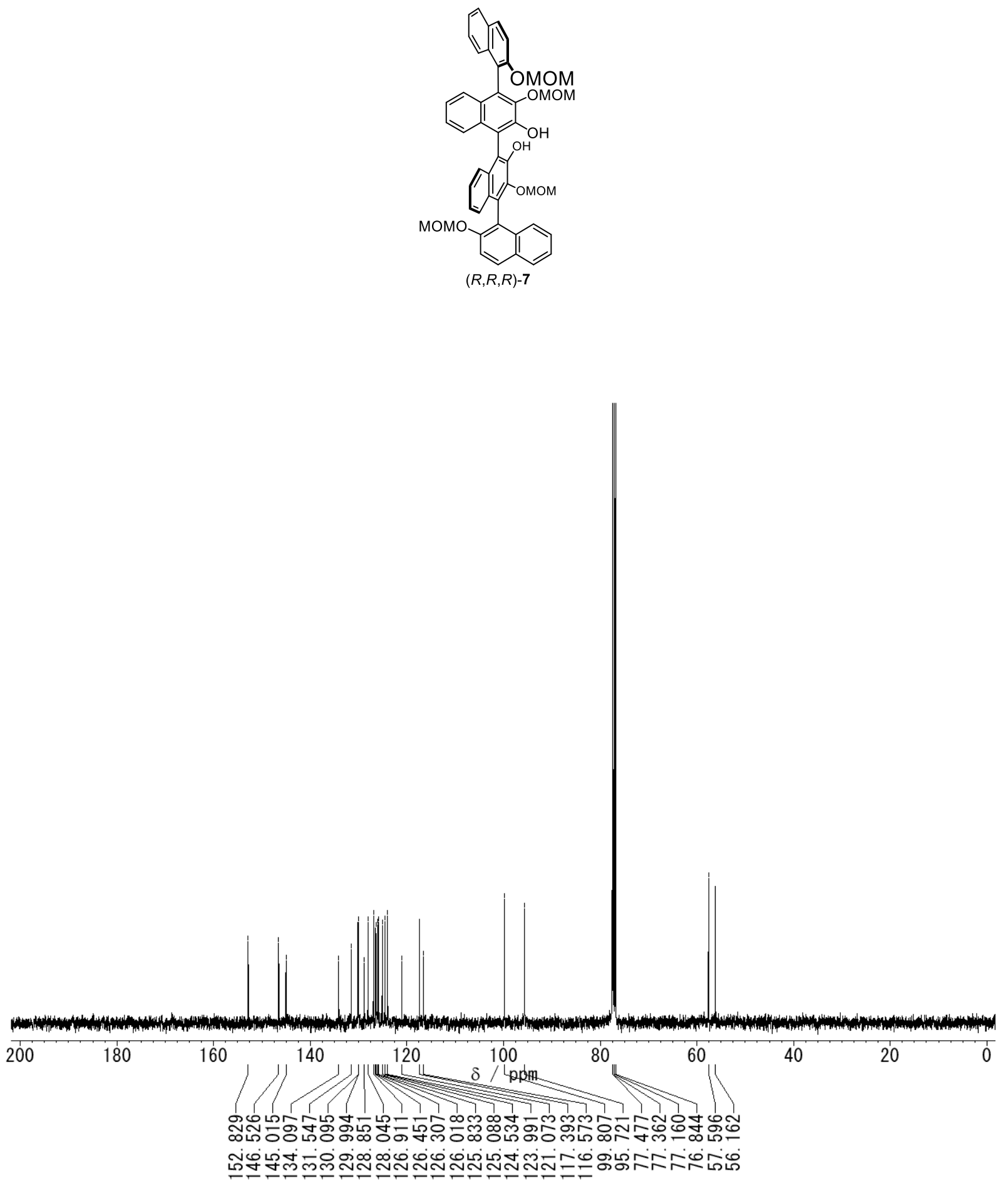

${ }^{13} \mathrm{C}$ NMR of $(R, R, R)-7\left(100 \mathrm{MHz}, \mathrm{CDCl}_{3}\right)$ 

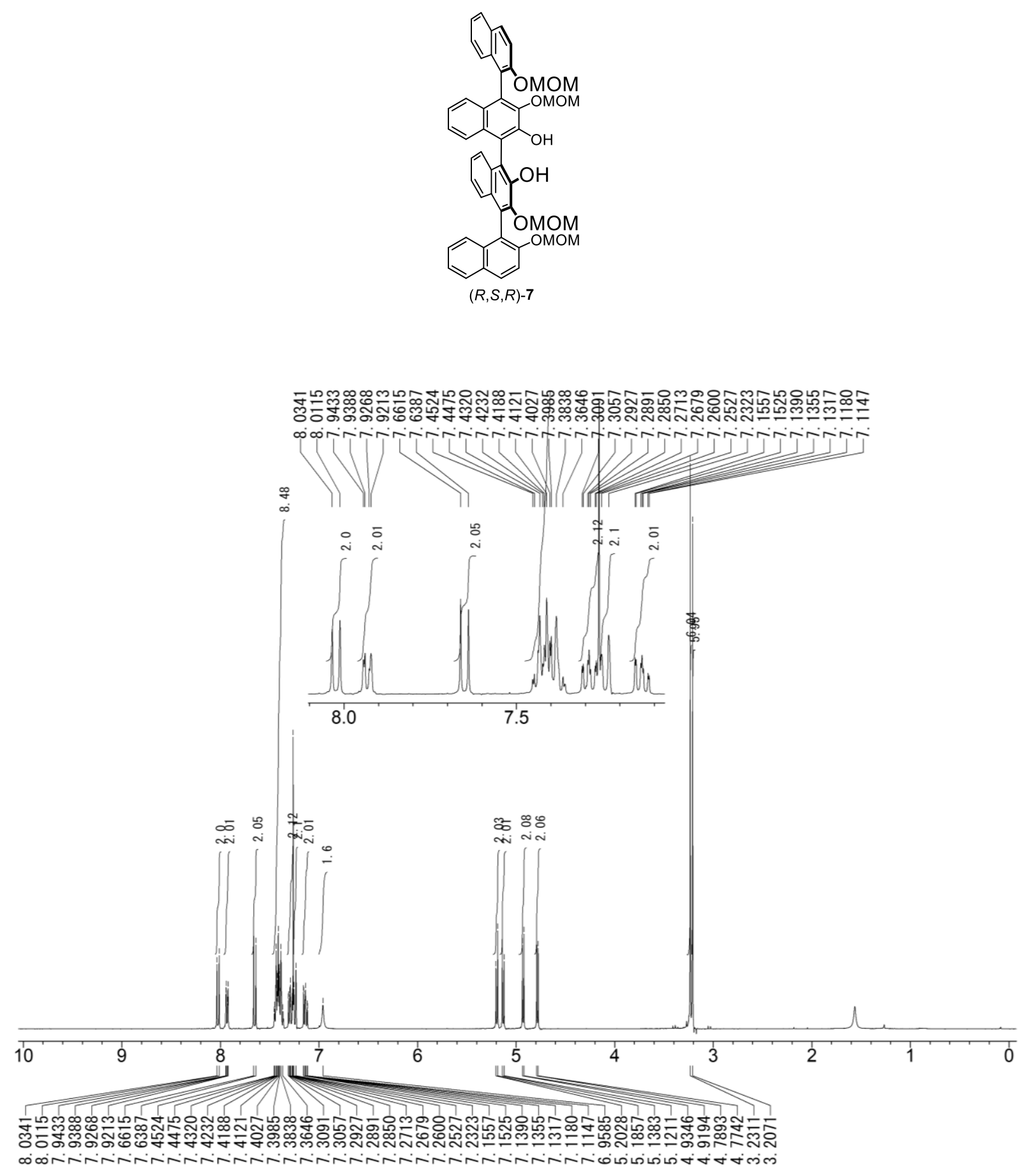

${ }^{1} \mathrm{H}$ NMR of $(R, S, R)-7\left(400 \mathrm{MHz}, \mathrm{CDCl}_{3}\right)$ 

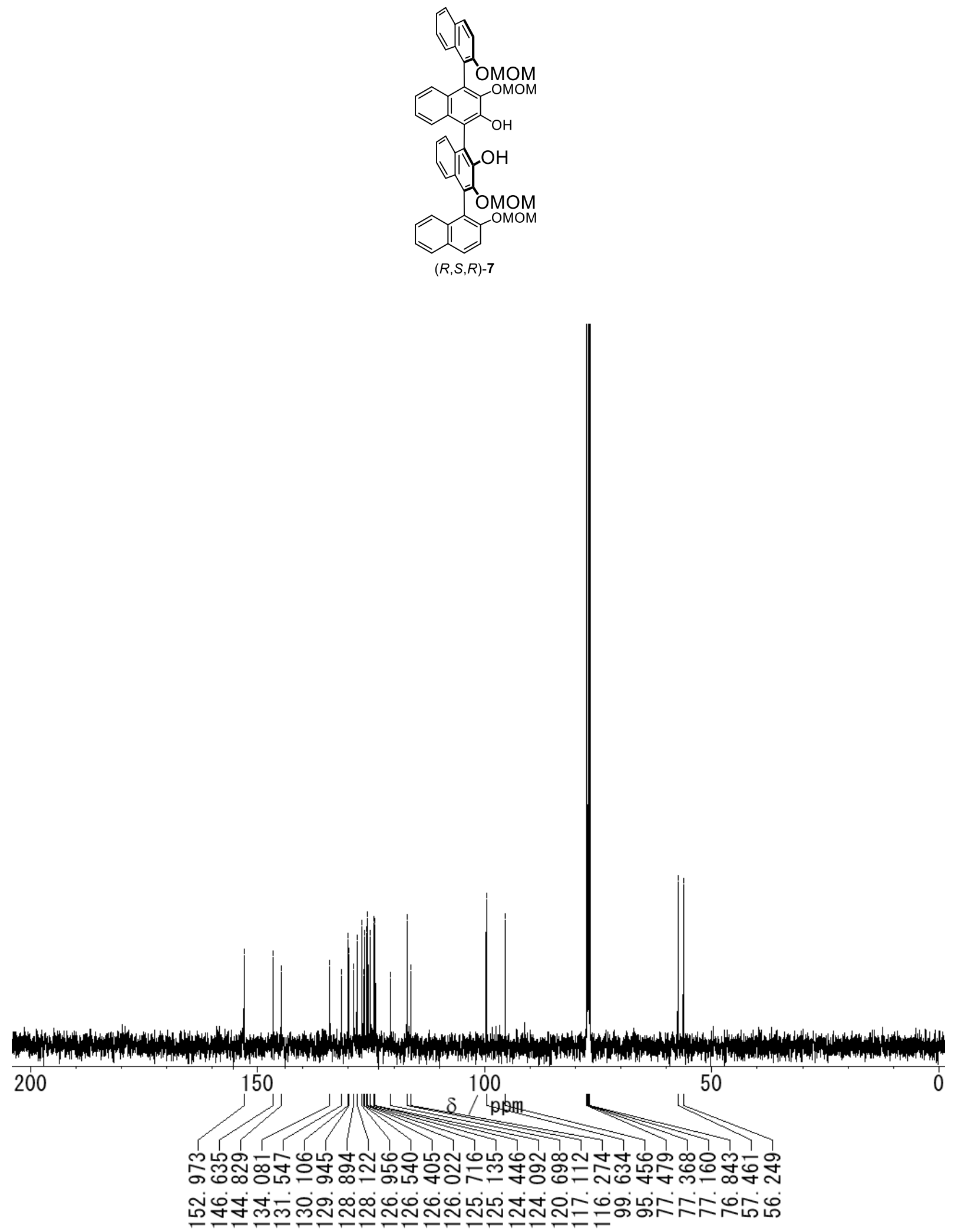

${ }^{13} \mathrm{C}$ NMR of $(R, S, R)-7\left(100 \mathrm{MHz}, \mathrm{CDCl}_{3}\right)$ 

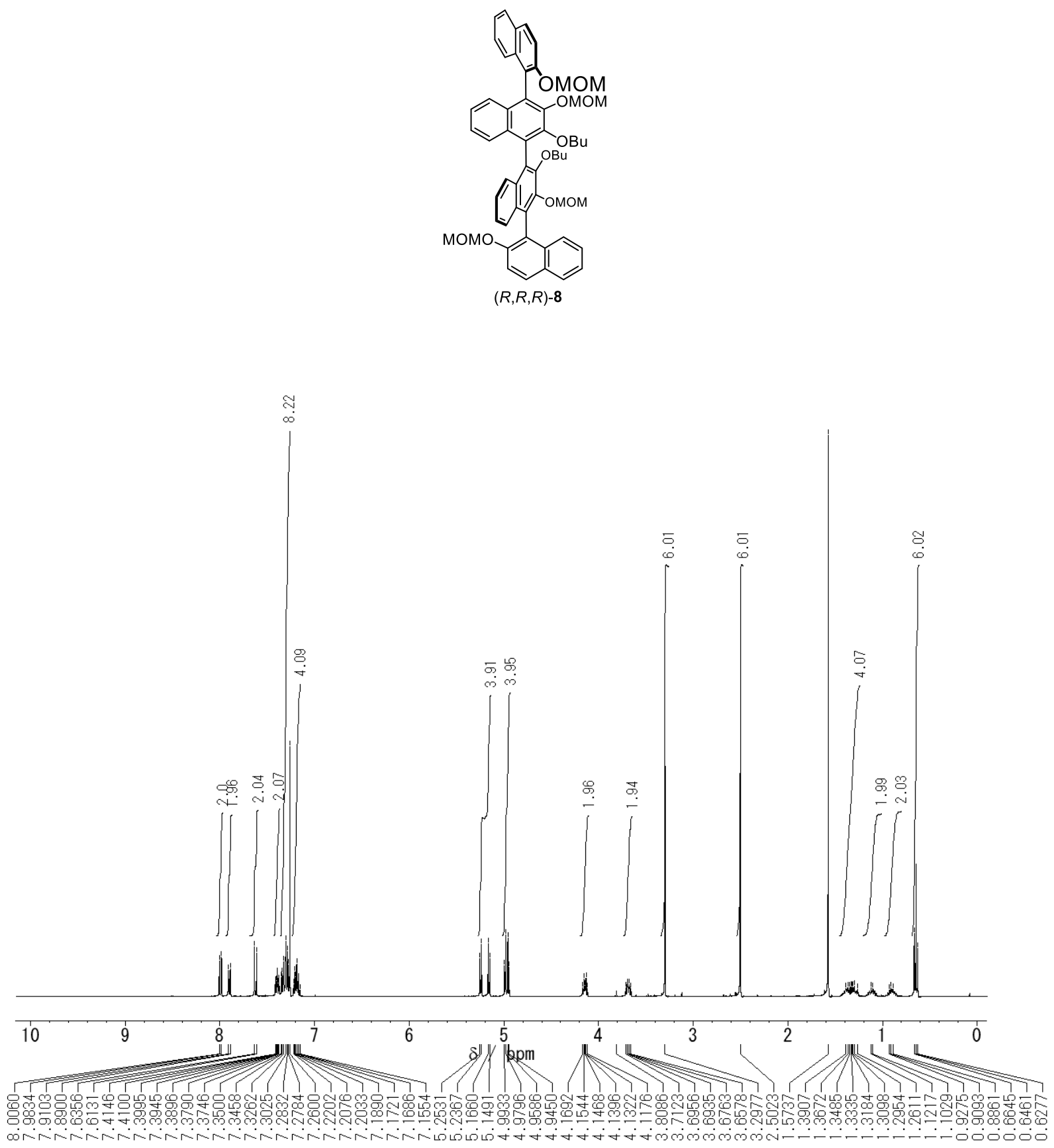

${ }^{1} \mathrm{H}$ NMR of $(R, R, R)-8\left(400 \mathrm{MHz}, \mathrm{CDCl}_{3}\right)$ 

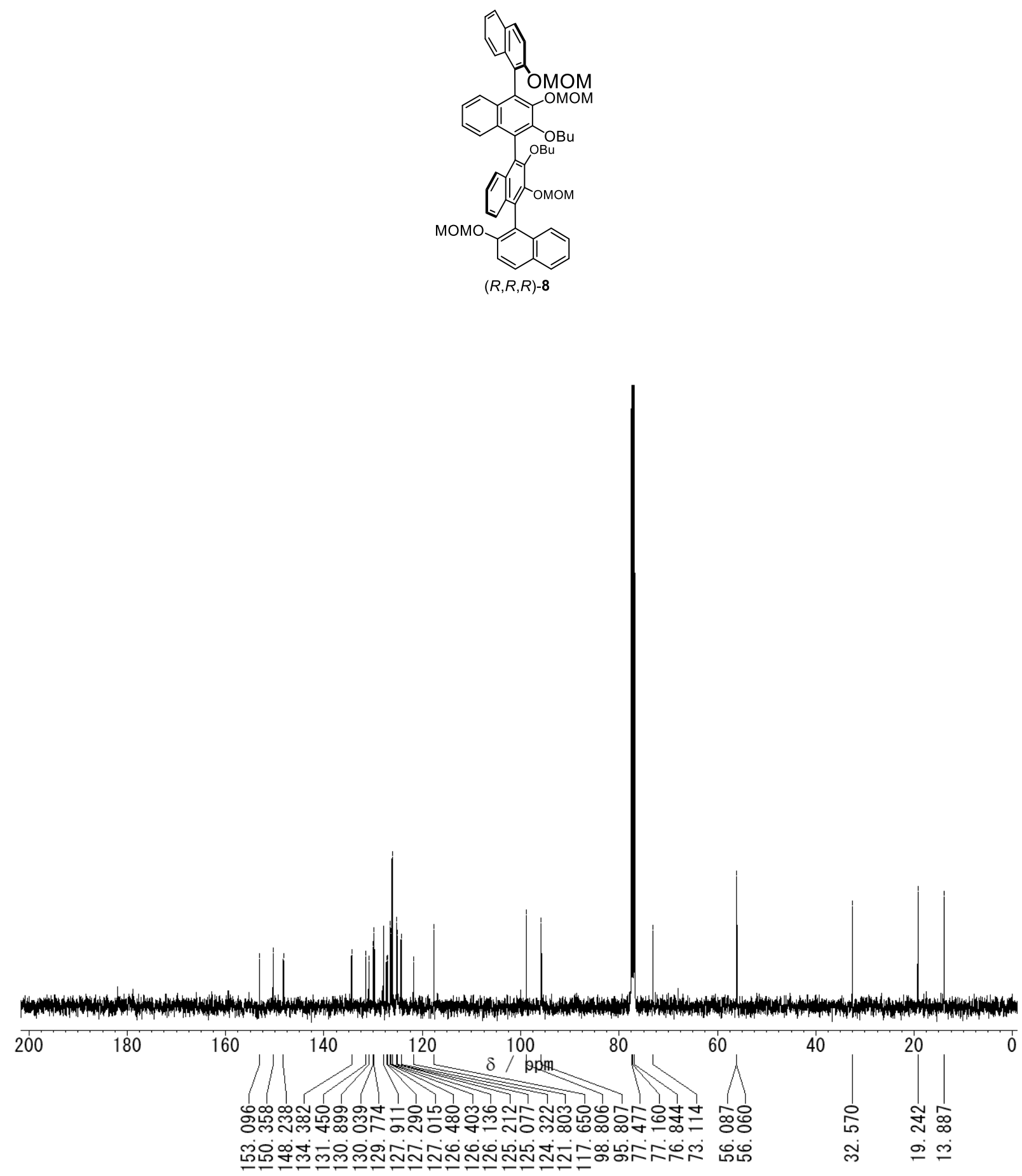

${ }^{13} \mathrm{C} \mathrm{NMR}$ of $(R, R, R)-\mathbf{8}\left(100 \mathrm{MHz}, \mathrm{CDCl}_{3}\right)$ 

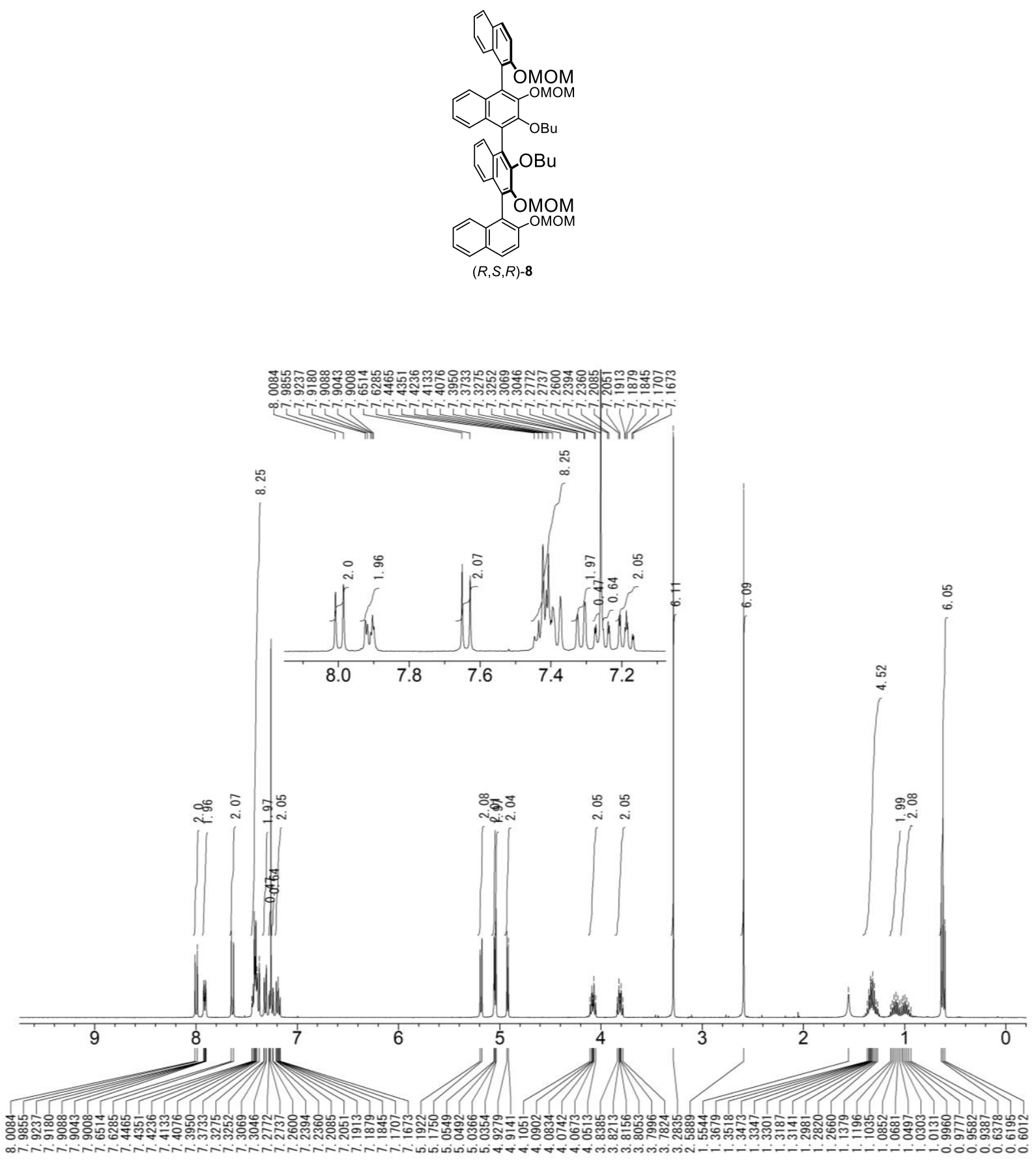

${ }^{1} \mathrm{H}$ NMR of $(R, S, R)-8\left(400 \mathrm{MHz}, \mathrm{CDCl}_{3}\right)$ 

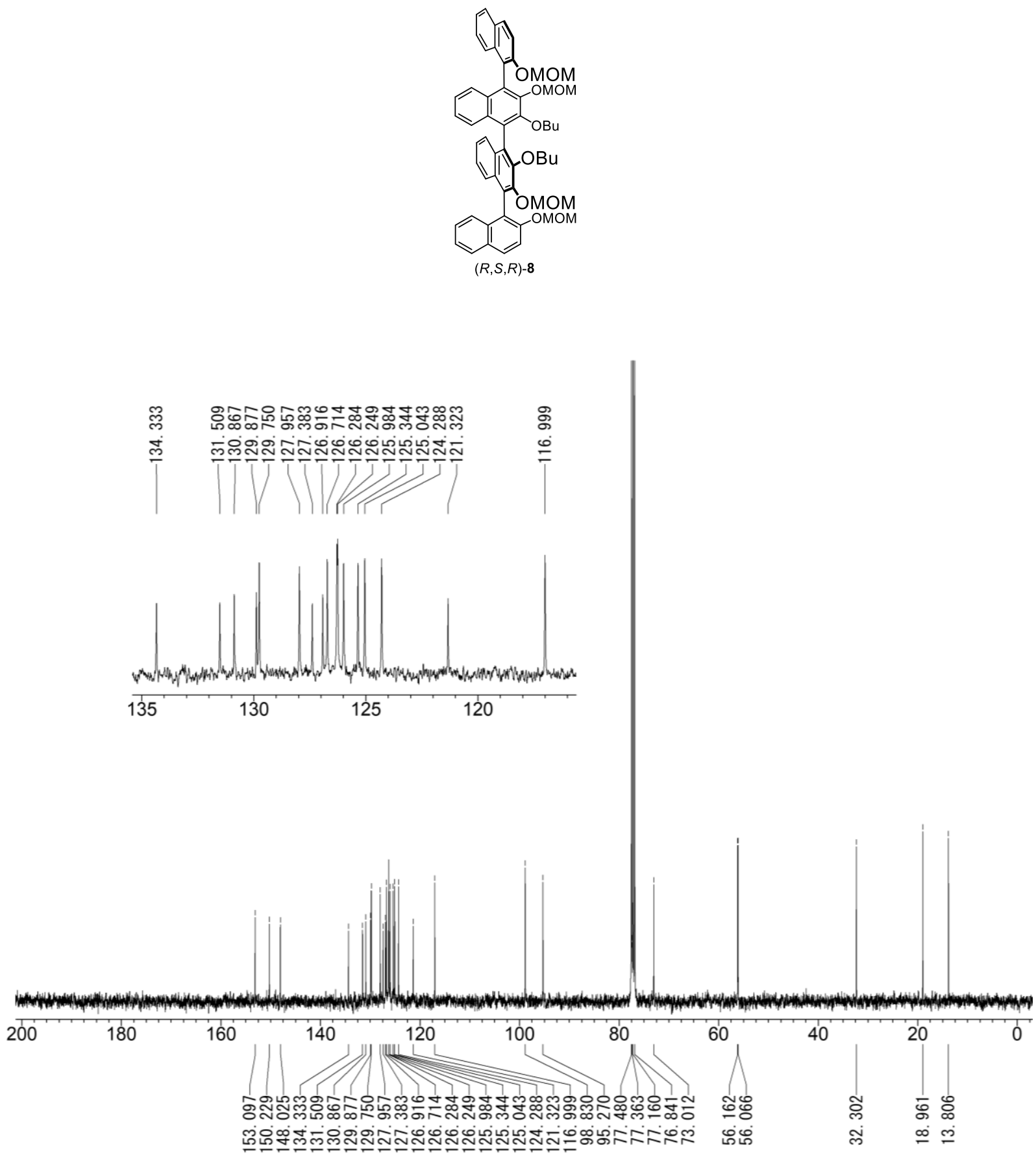

${ }^{13} \mathrm{C}$ NMR of $(R, S, R)-8\left(100 \mathrm{MHz}, \mathrm{CDCl}_{3}\right)$ 

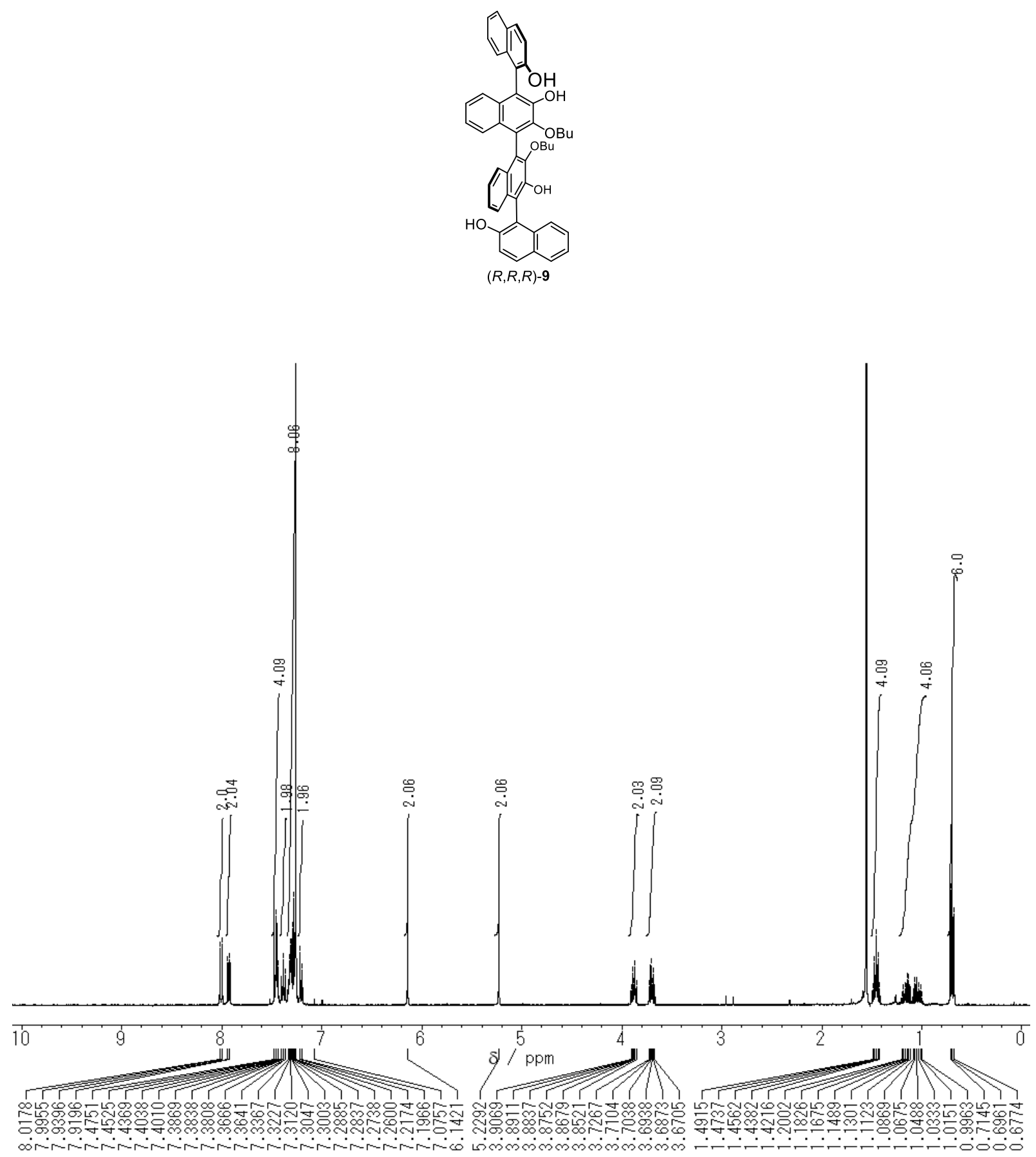

${ }^{1} \mathrm{H}$ NMR of $(R, R, R)-9\left(400 \mathrm{MHz}, \mathrm{CDCl}_{3}\right)$ 

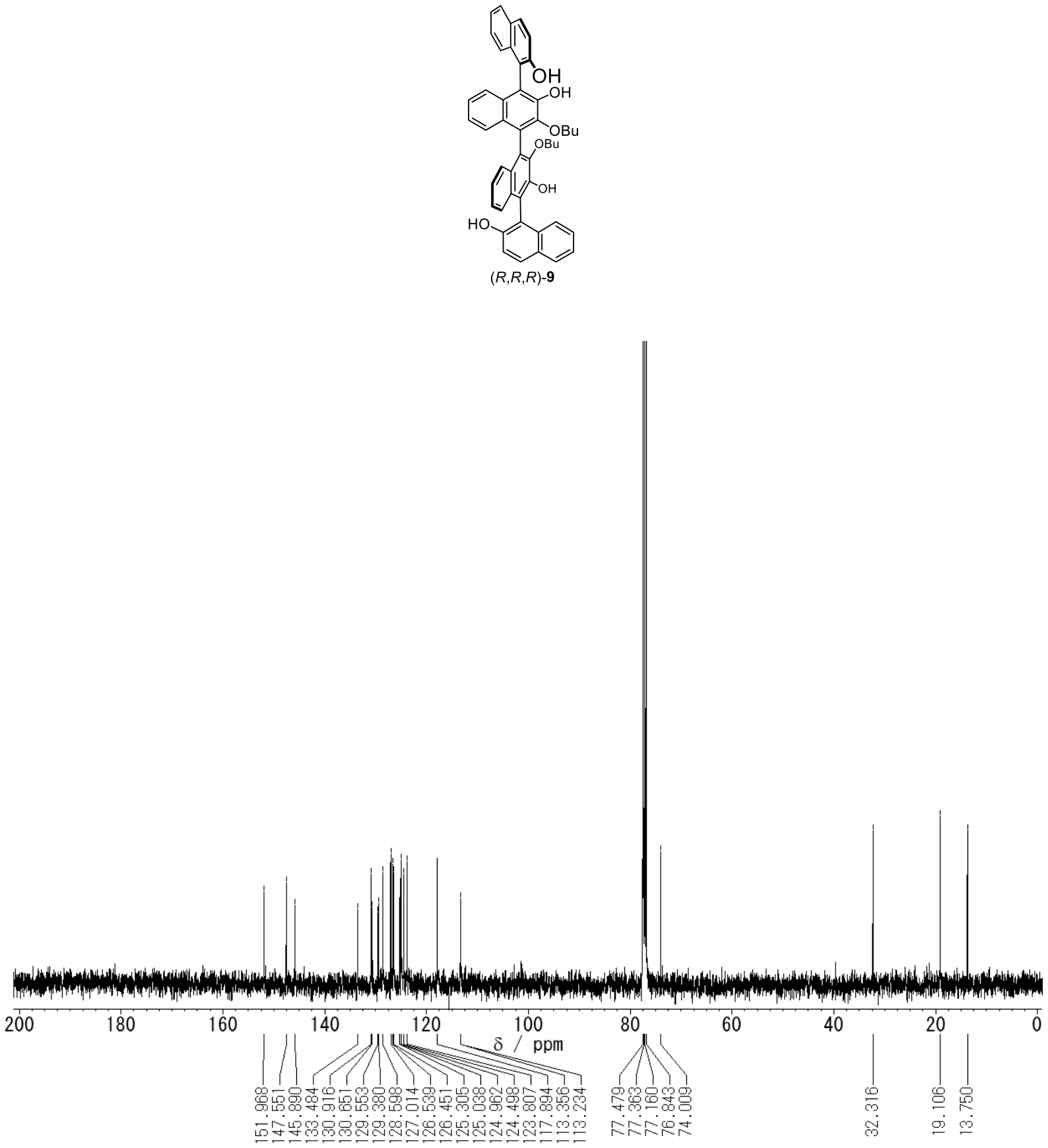

${ }^{13} \mathrm{C}$ NMR of $(R, R, R)-9\left(100 \mathrm{MHz}, \mathrm{CDCl}_{3}\right)$ 

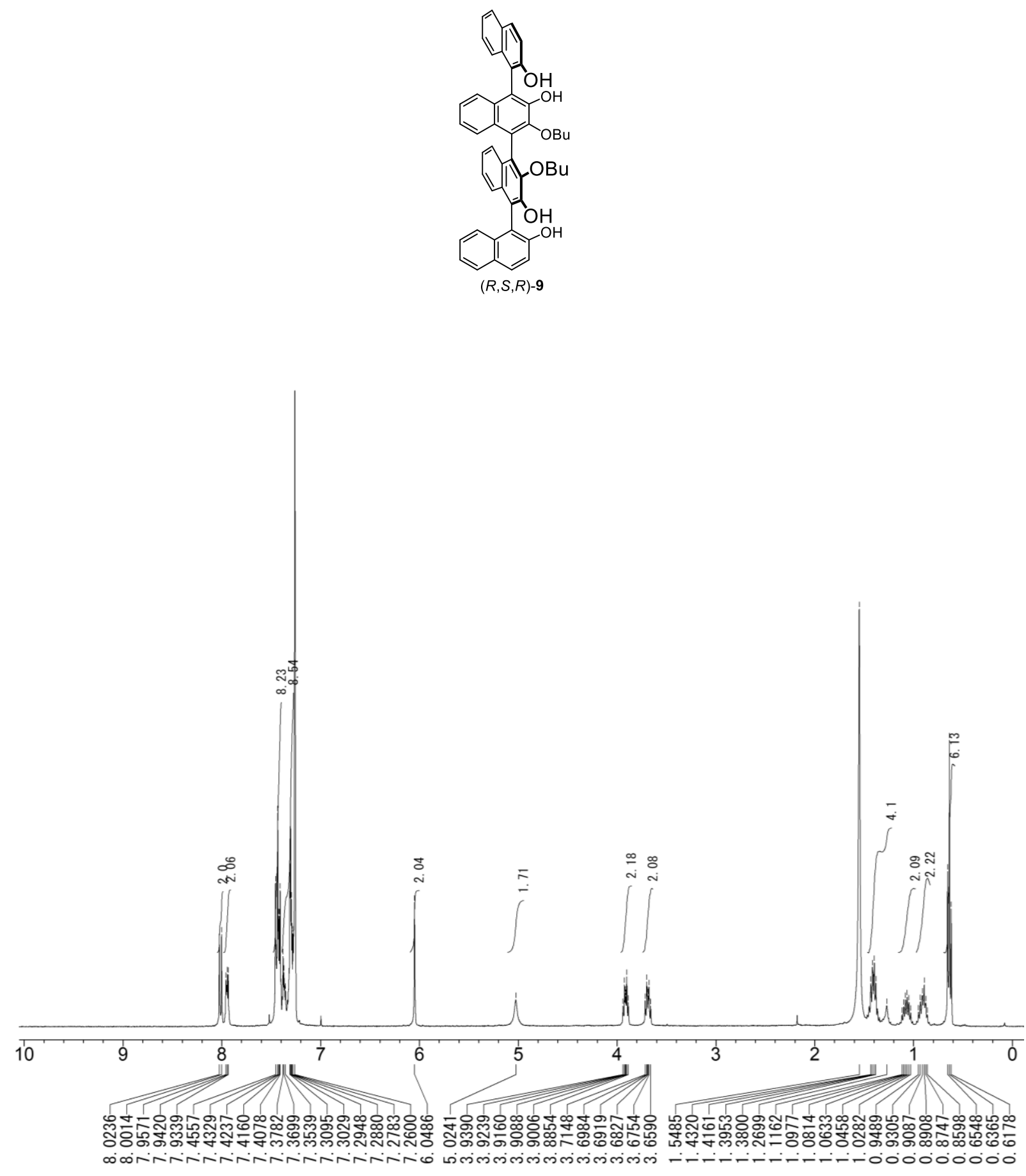

${ }^{1} \mathrm{H}$ NMR of $(R, S, R)-9\left(400 \mathrm{MHz}, \mathrm{CDCl}_{3}\right)$ 

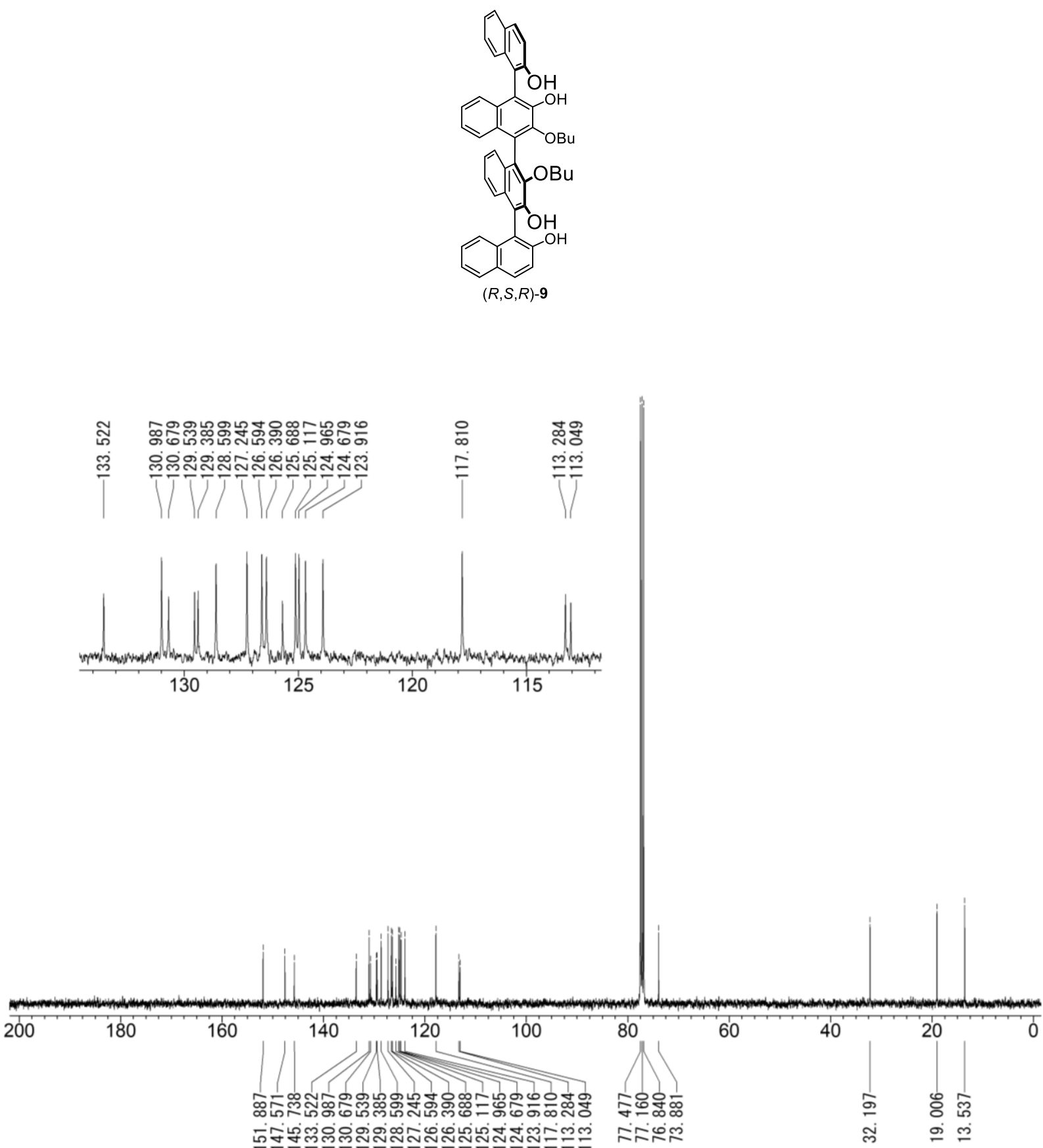

${ }^{13} \mathrm{C}$ NMR of $(R, S, R)-9\left(100 \mathrm{MHz}, \mathrm{CDCl}_{3}\right)$ 

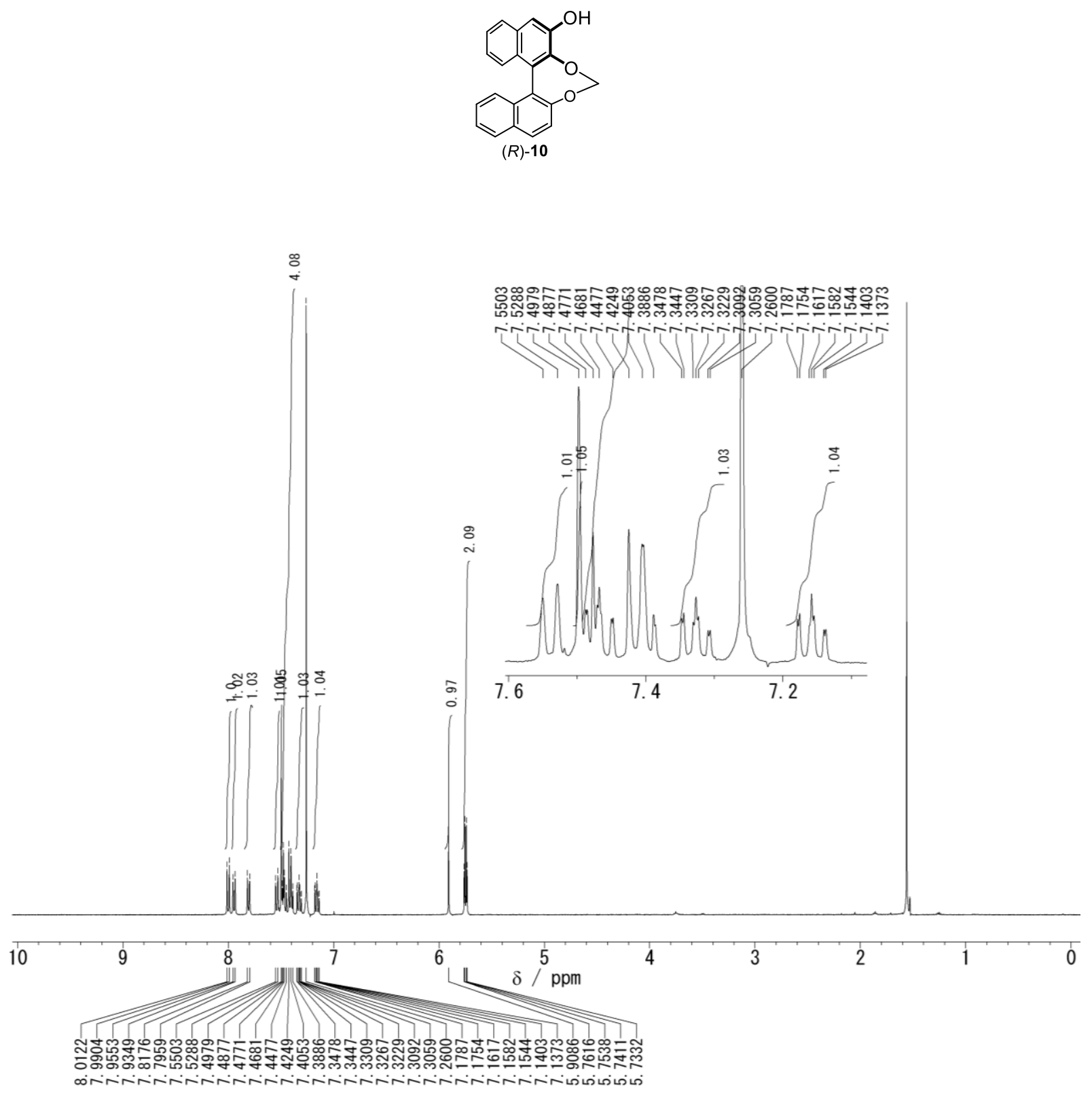

${ }^{1} \mathrm{H}$ NMR of $(R)-10\left(400 \mathrm{MHz}, \mathrm{CDCl}_{3}\right)$ 

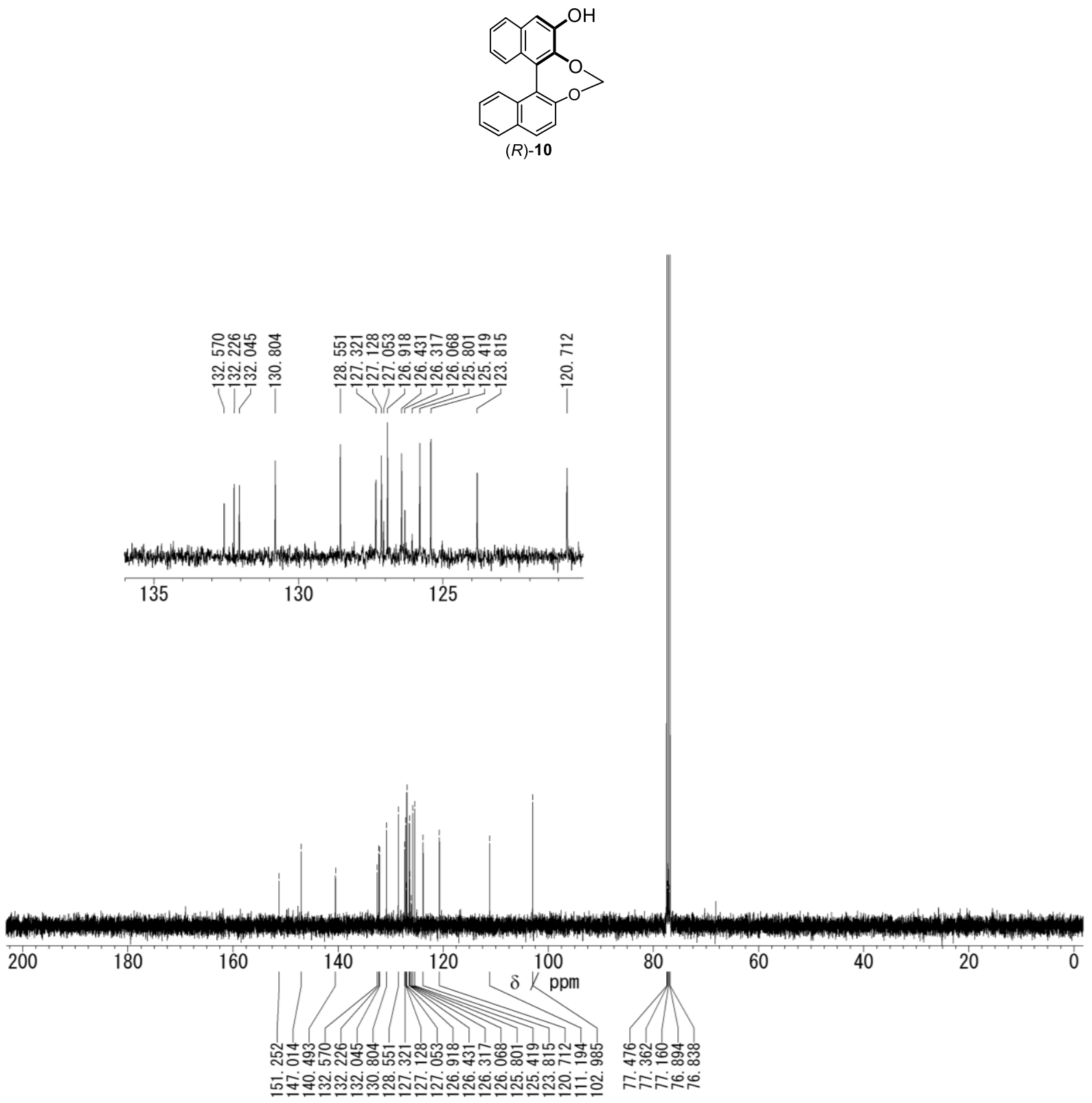

${ }^{13} \mathrm{C}$ NMR of $(R)-\mathbf{1 0}\left(100 \mathrm{MHz}, \mathrm{CDCl}_{3}\right)$ 

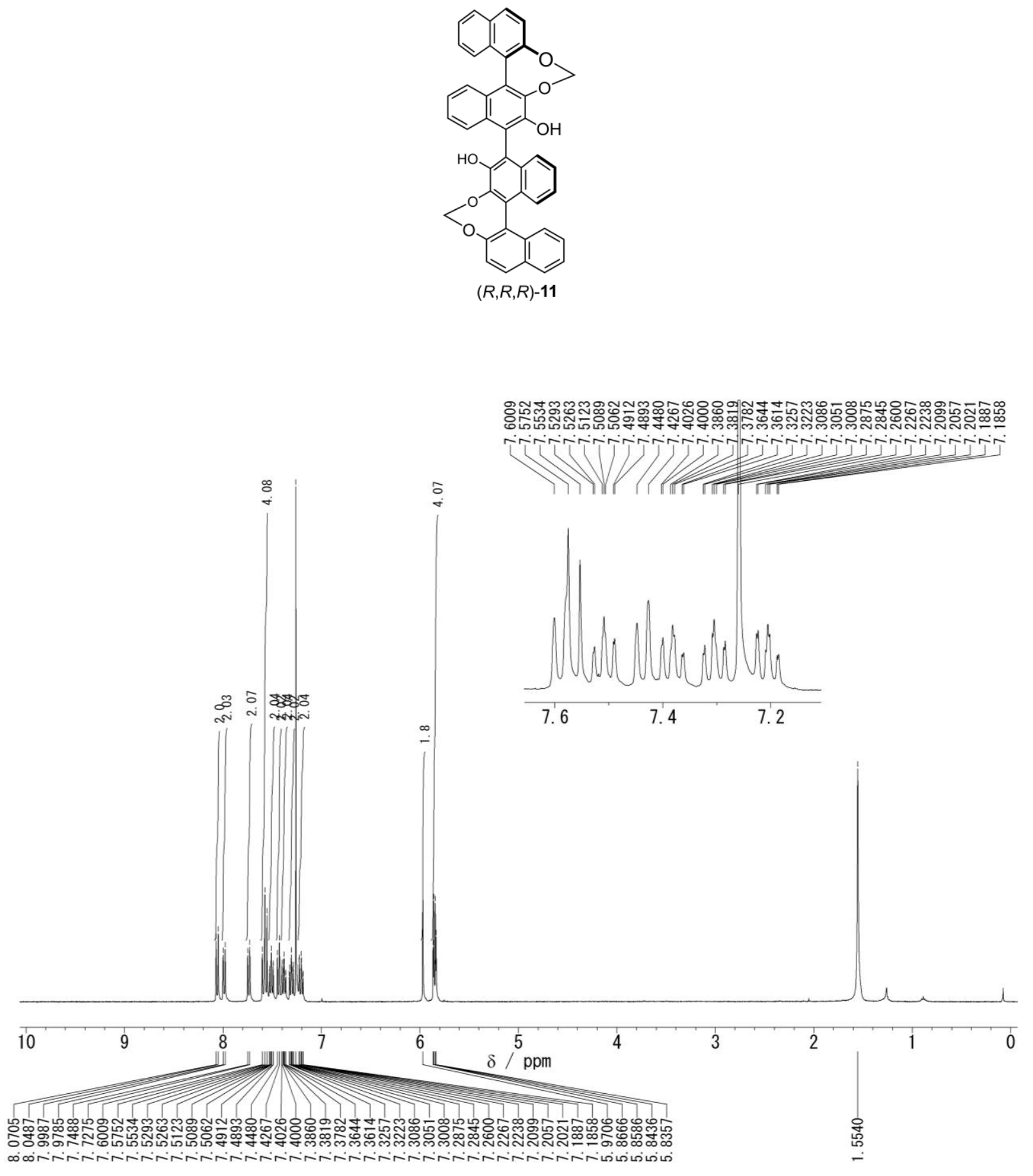

${ }^{1} \mathrm{H}$ NMR of $(R, R, R)-11\left(400 \mathrm{MHz}, \mathrm{CDCl}_{3}\right)$ 


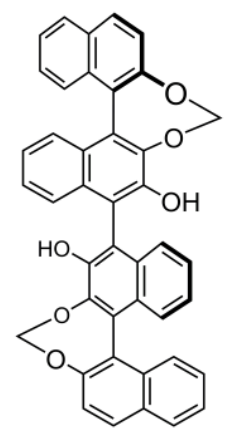

$(R, R, R)-11$
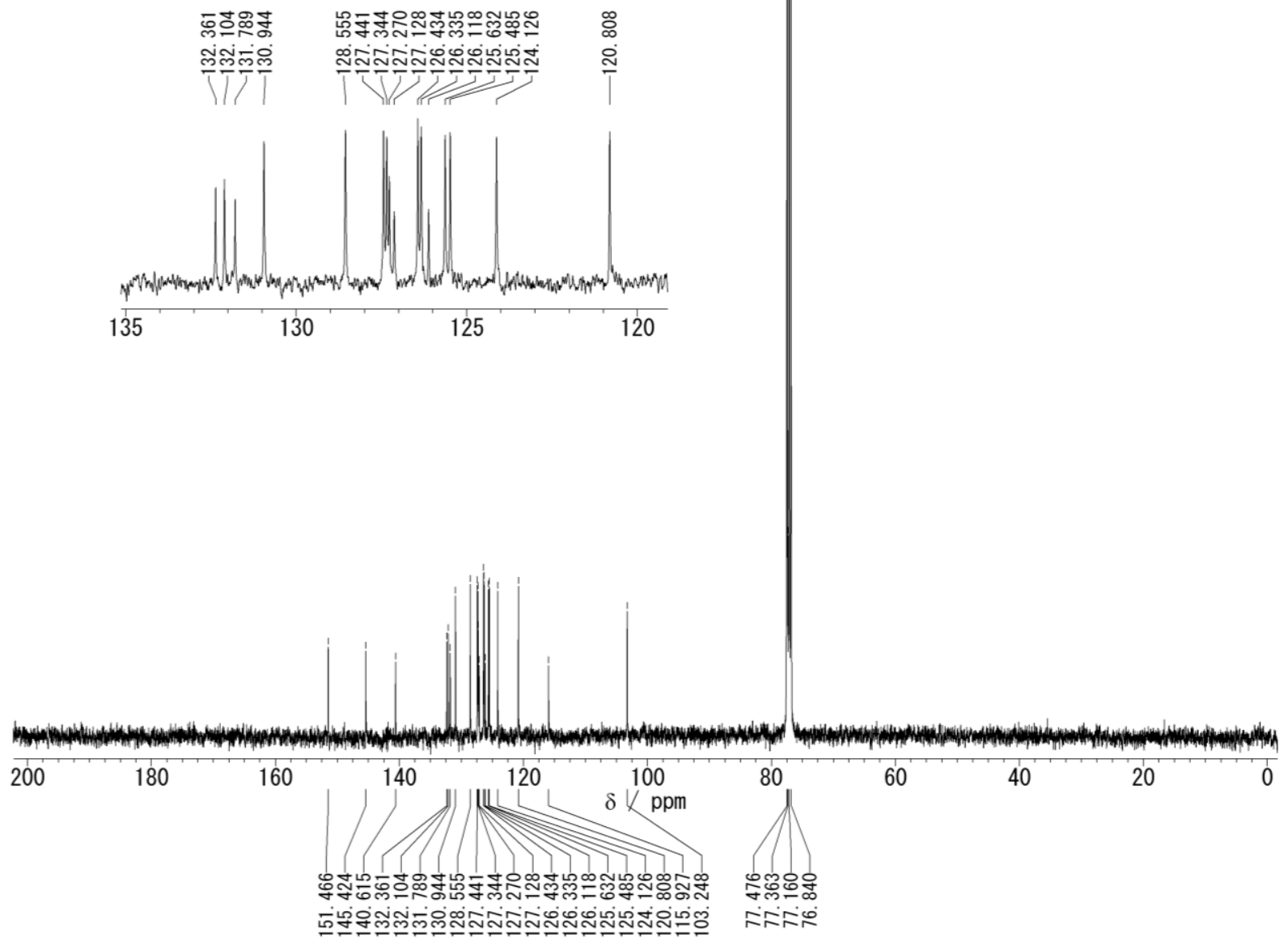

${ }^{13} \mathrm{C}$ NMR of $(R, R, R)-\mathbf{1 1}\left(100 \mathrm{MHz}, \mathrm{CDCl}_{3}\right)$ 

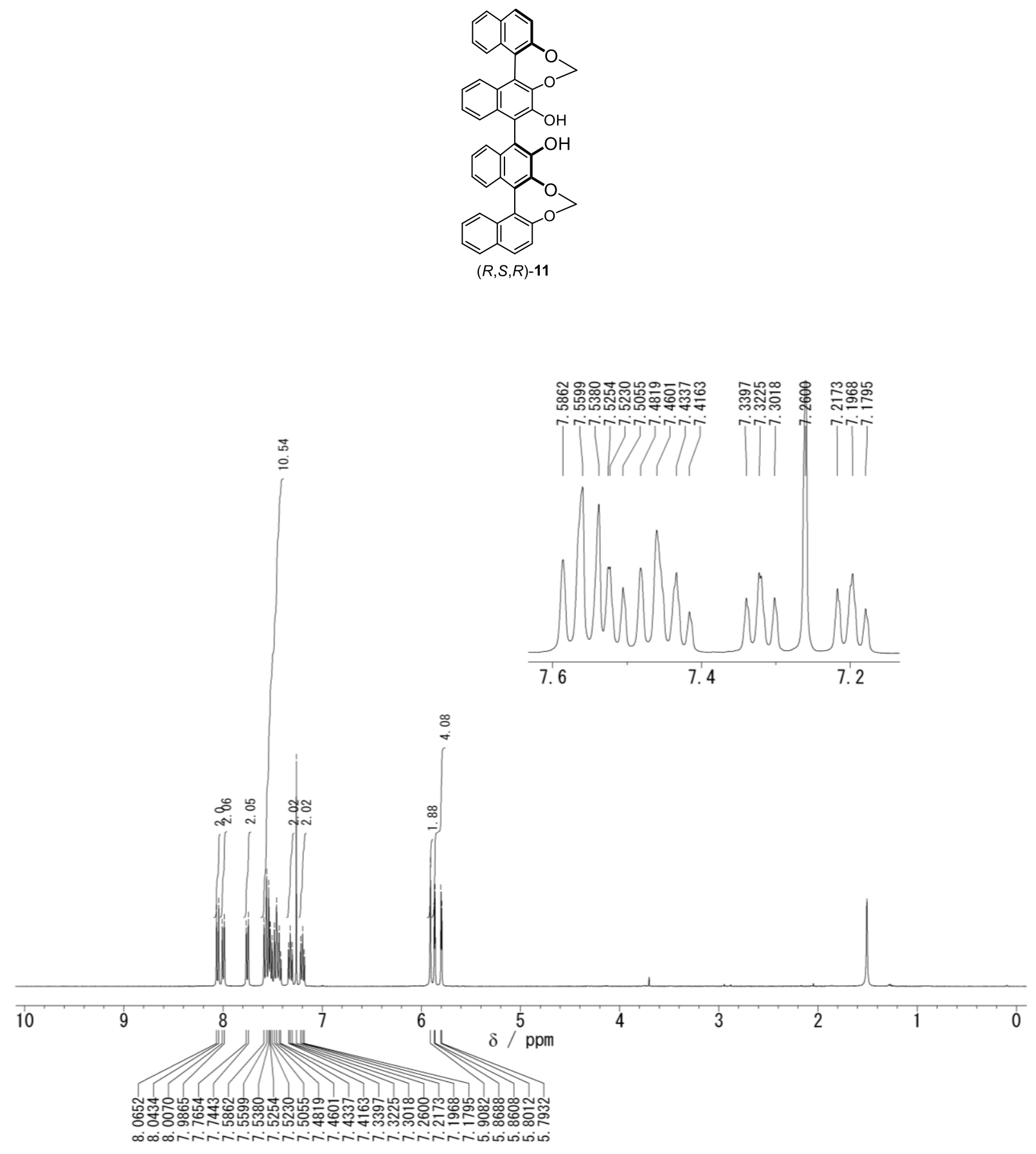

${ }^{1} \mathrm{H}$ NMR of $(R, S, R)-\mathbf{1 1}\left(400 \mathrm{MHz}, \mathrm{CDCl}_{3}\right)$ 


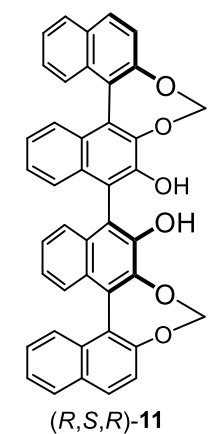

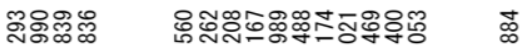

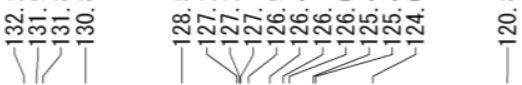
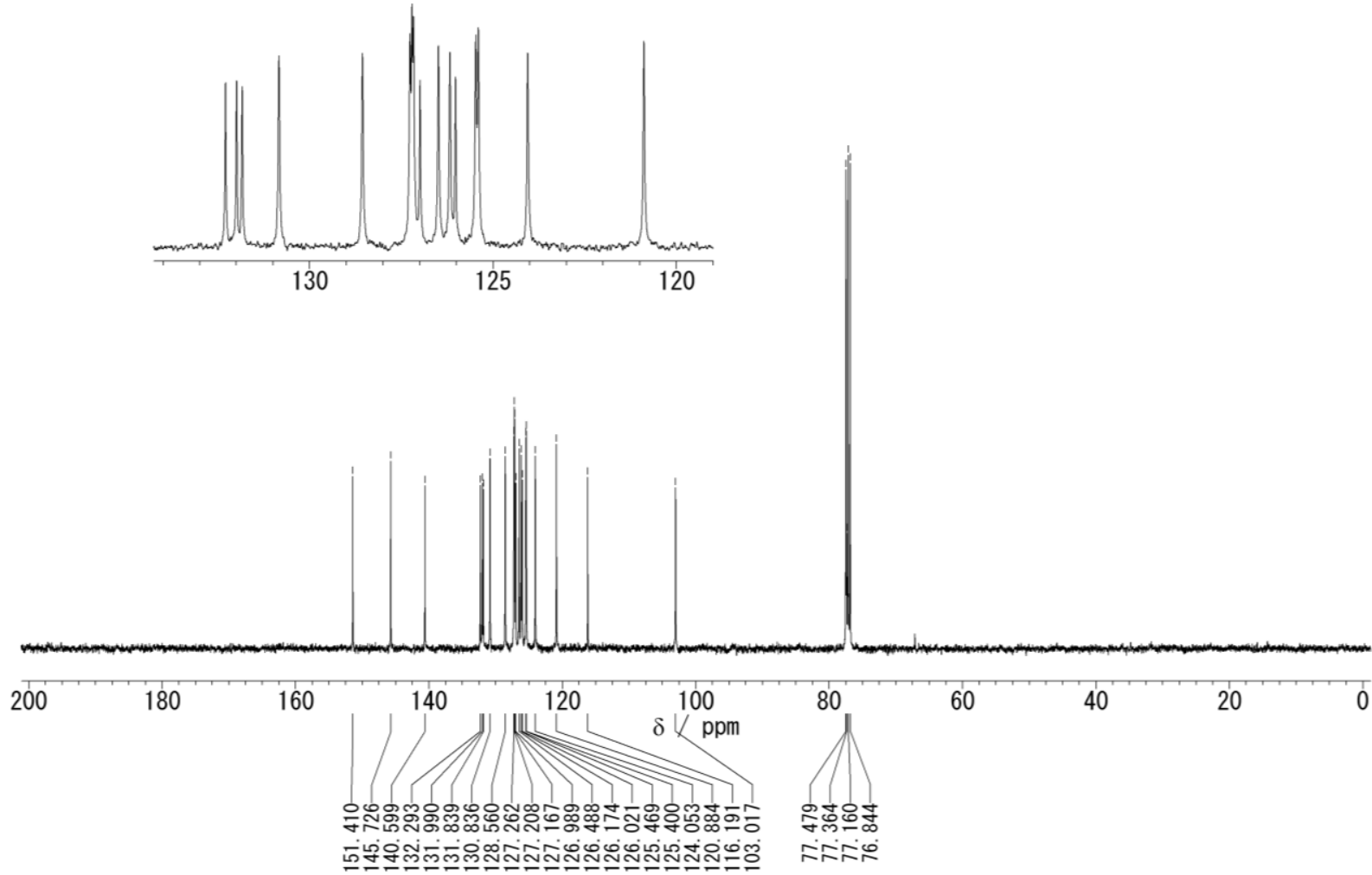

${ }^{13} \mathrm{C}$ NMR of $(R, S, R)-\mathbf{1 1}\left(100 \mathrm{MHz}, \mathrm{CDCl}_{3}\right)$ 

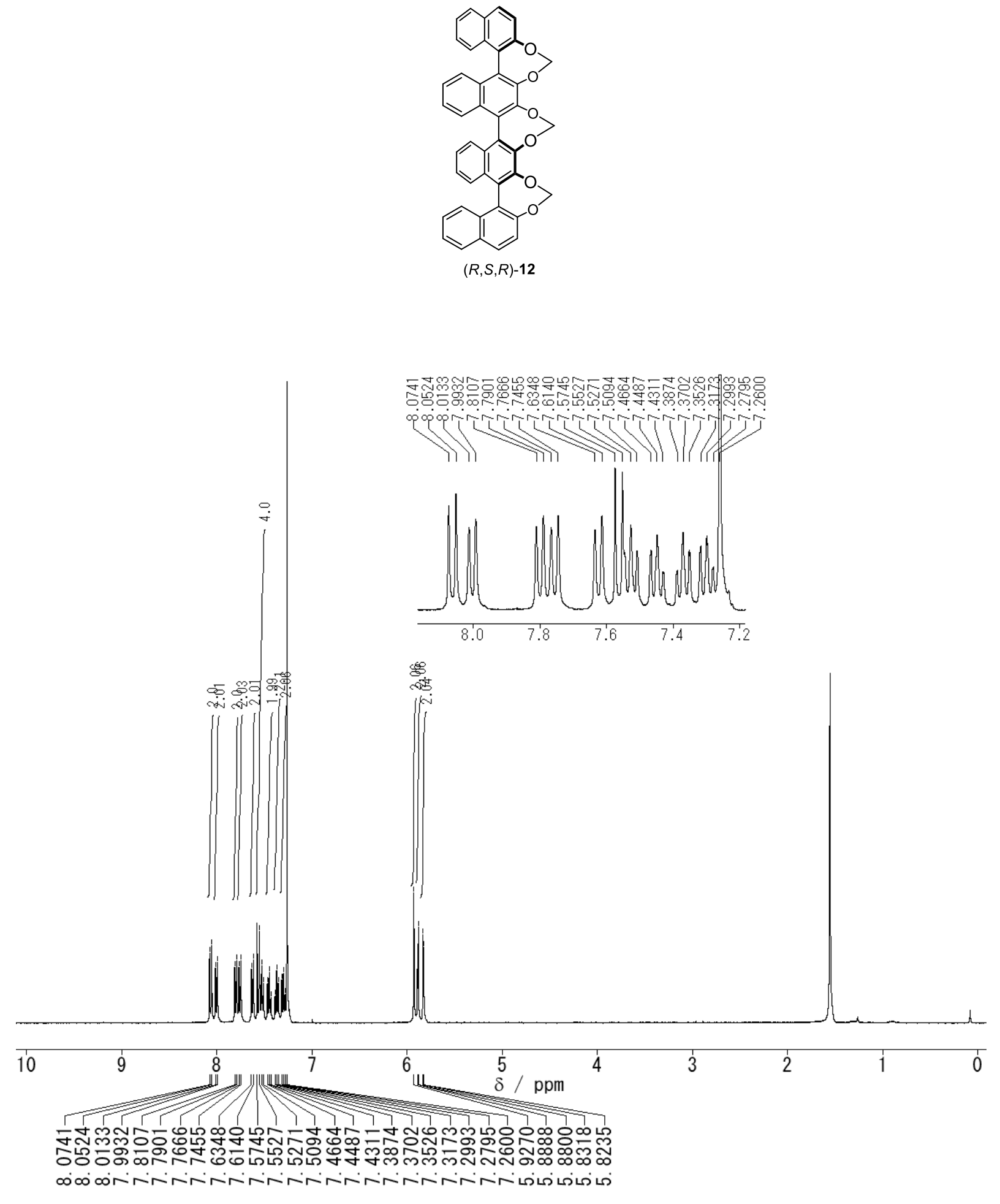

${ }^{1} \mathrm{H} \mathrm{NMR}$ of $(R, S, R)-12\left(400 \mathrm{MHz}, \mathrm{CDCl}_{3}\right)$ 

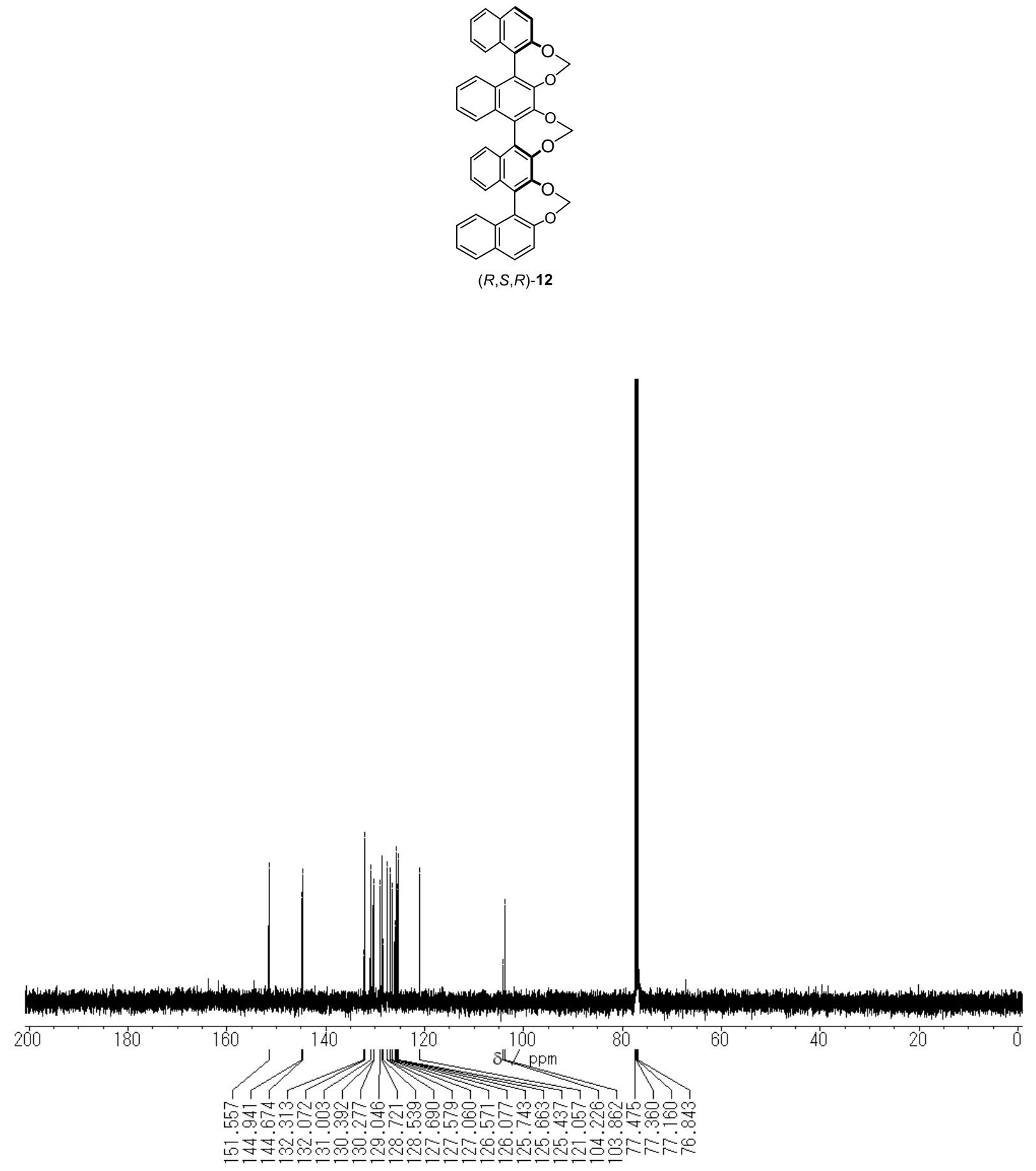

${ }^{13} \mathrm{C}$ NMR of $(R, S, R)-\mathbf{1 2}\left(100 \mathrm{MHz}, \mathrm{CDCl}_{3}\right)$ 

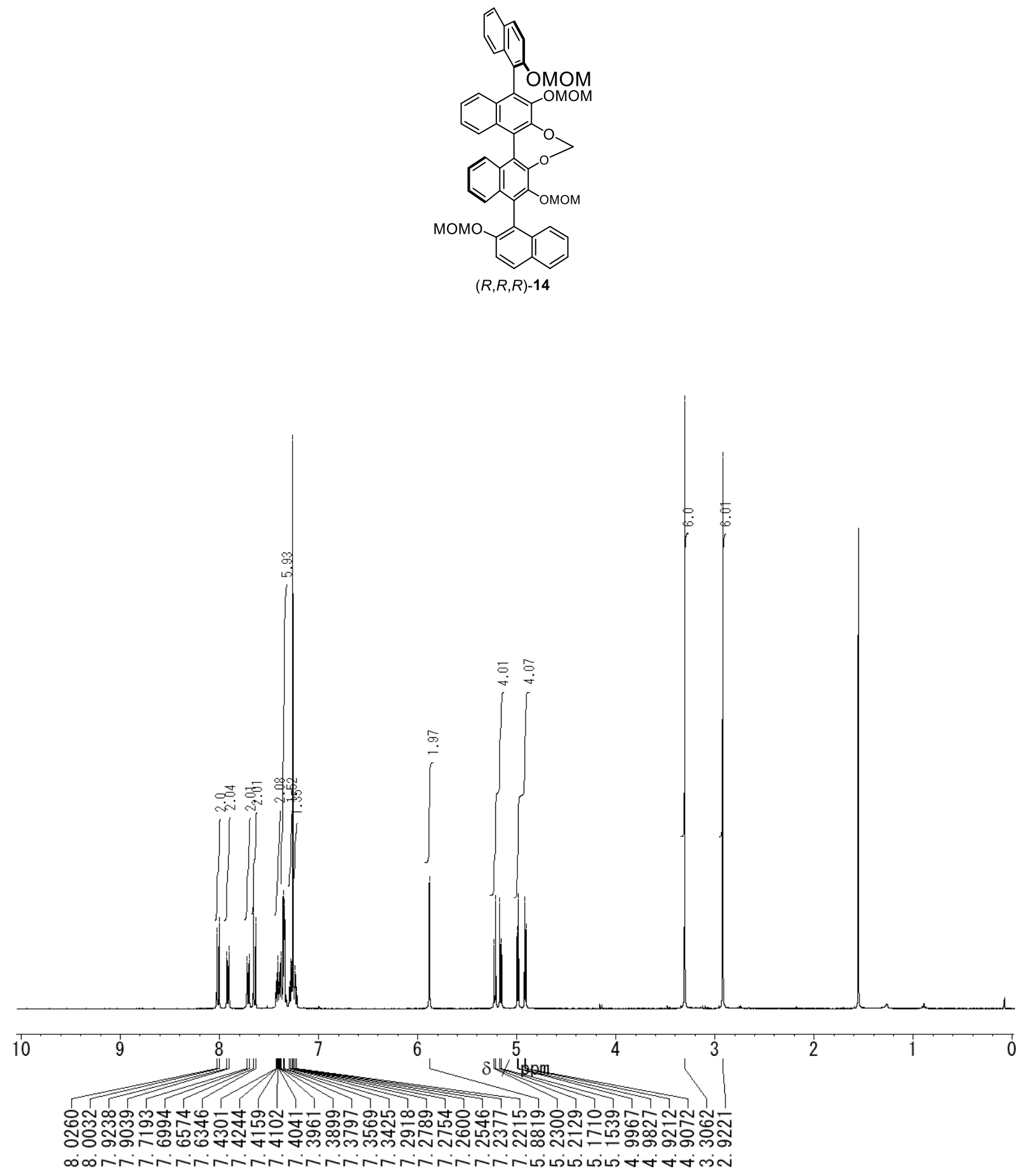

${ }^{1} \mathrm{H}$ NMR of $(R, R, R)-14\left(400 \mathrm{MHz}, \mathrm{CDCl}_{3}\right)$ 

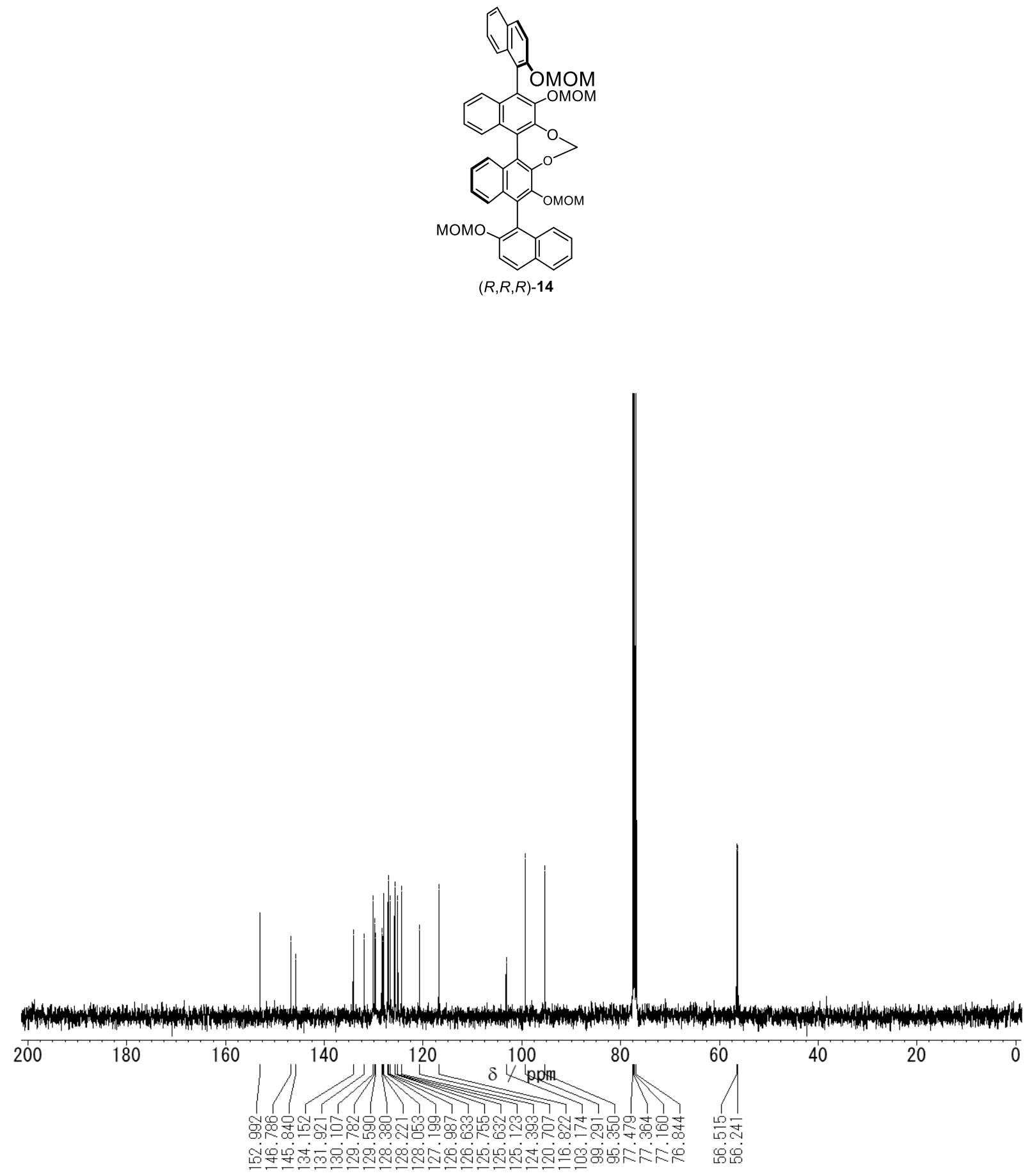

${ }^{13} \mathrm{C}$ NMR of $(R, R, R)-\mathbf{1 4}\left(100 \mathrm{MHz}, \mathrm{CDCl}_{3}\right)$ 

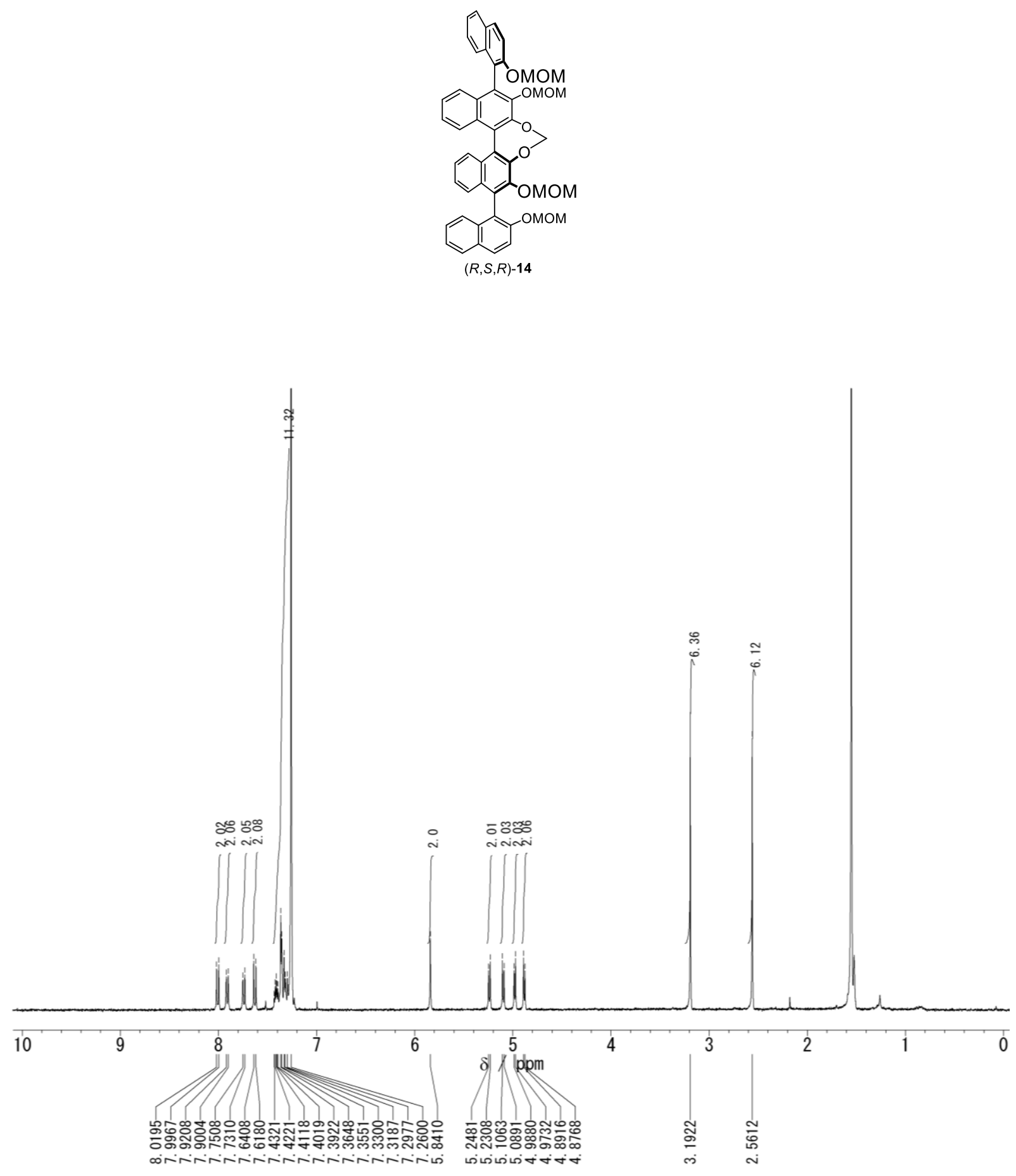

${ }^{1} \mathrm{H}$ NMR of $(R, S, R)-\mathbf{1 4}\left(400 \mathrm{MHz}, \mathrm{CDCl}_{3}\right)$ 

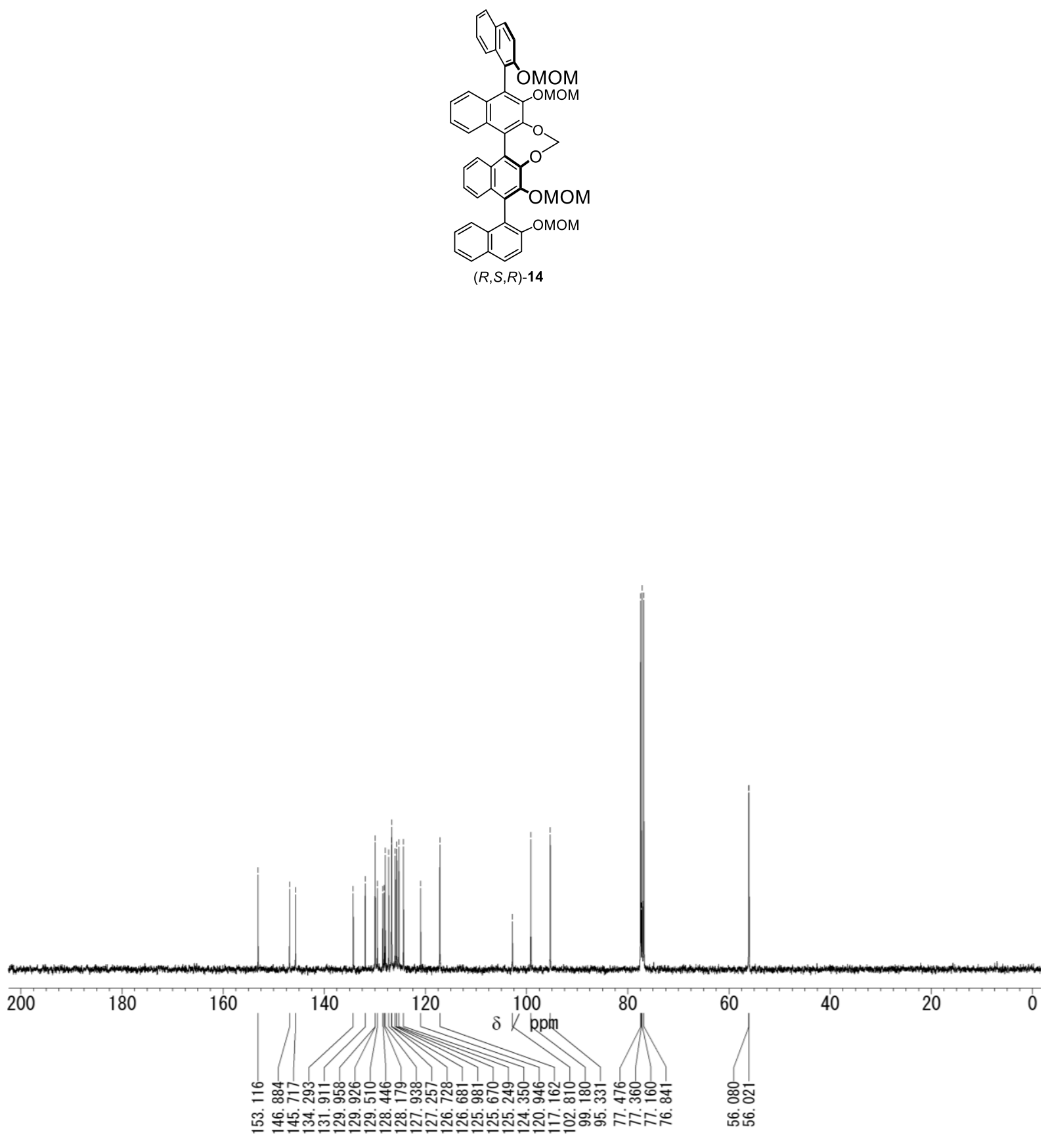

${ }^{13} \mathrm{C}$ NMR of $(R, S, R)-\mathbf{1 4}\left(100 \mathrm{MHz}, \mathrm{CDCl}_{3}\right)$ 

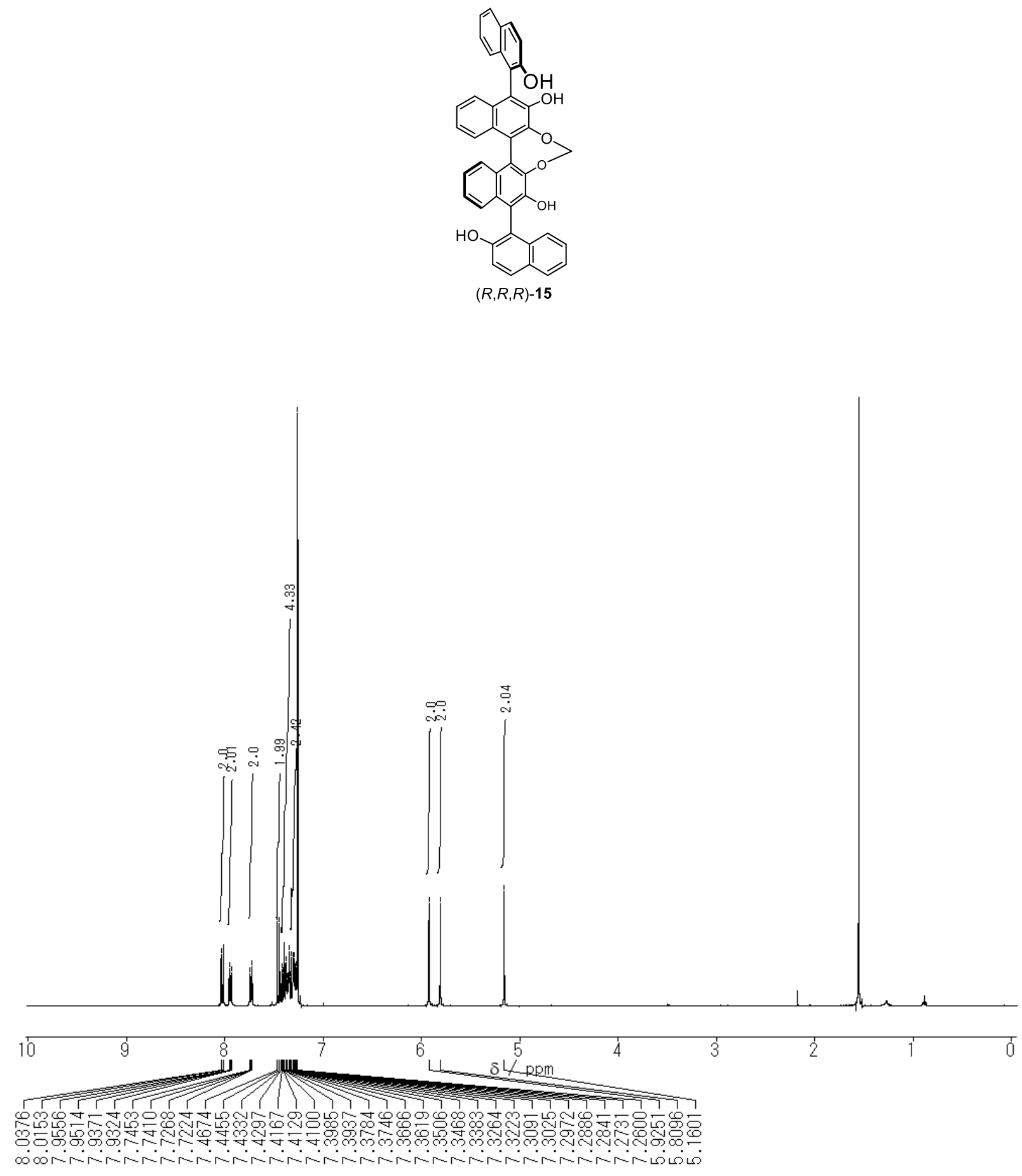

${ }^{1} \mathrm{H}$ NMR of $(R, R, R)-15\left(400 \mathrm{MHz}, \mathrm{CDCl}_{3}\right)$ 

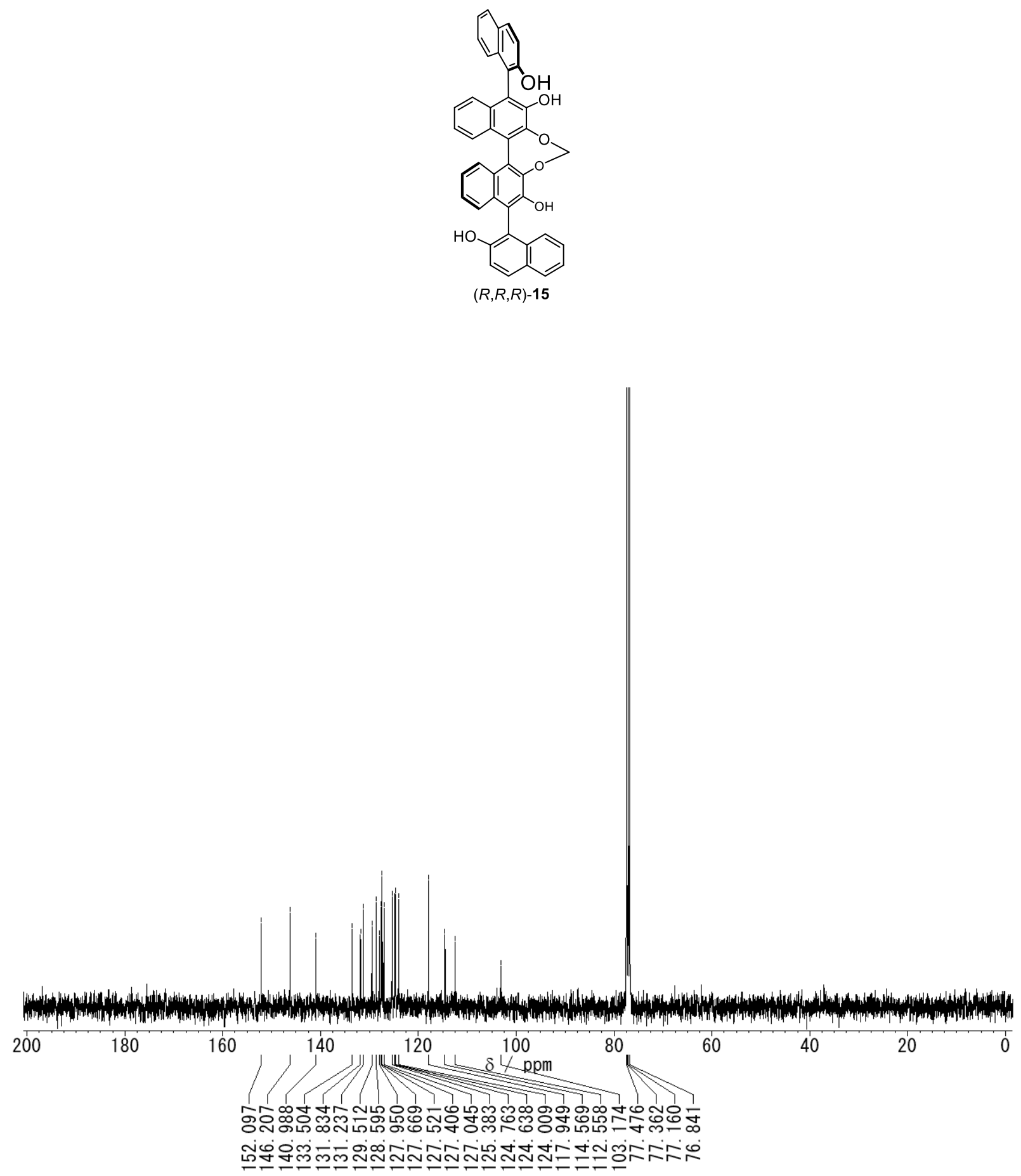

${ }^{13} \mathrm{C}$ NMR of $(R, R, R)-\mathbf{1 5}\left(100 \mathrm{MHz}, \mathrm{CDCl}_{3}\right)$ 

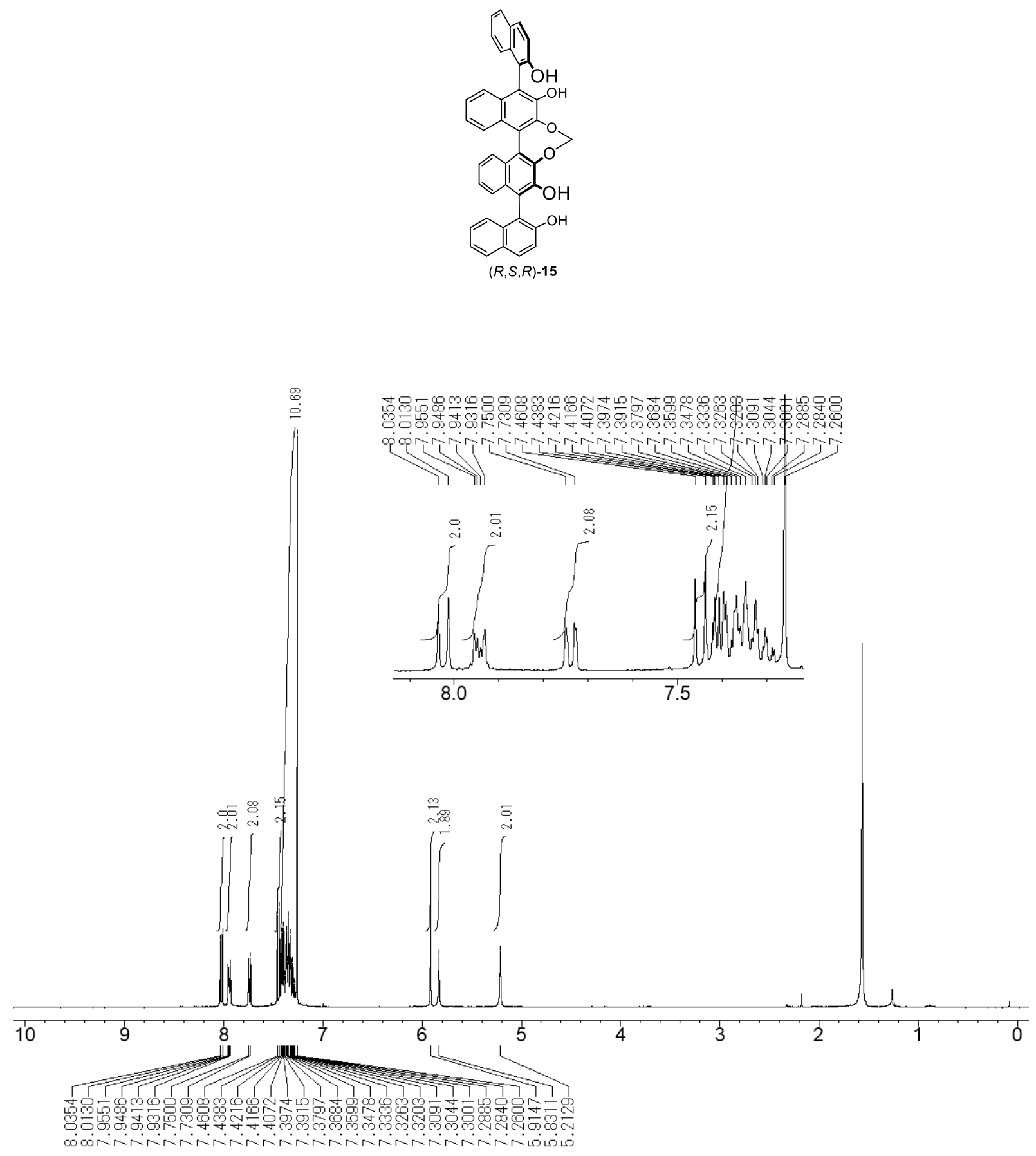

${ }^{1} \mathrm{H}$ NMR of $(R, S, R)-15\left(400 \mathrm{MHz}, \mathrm{CDCl}_{3}\right)$ 

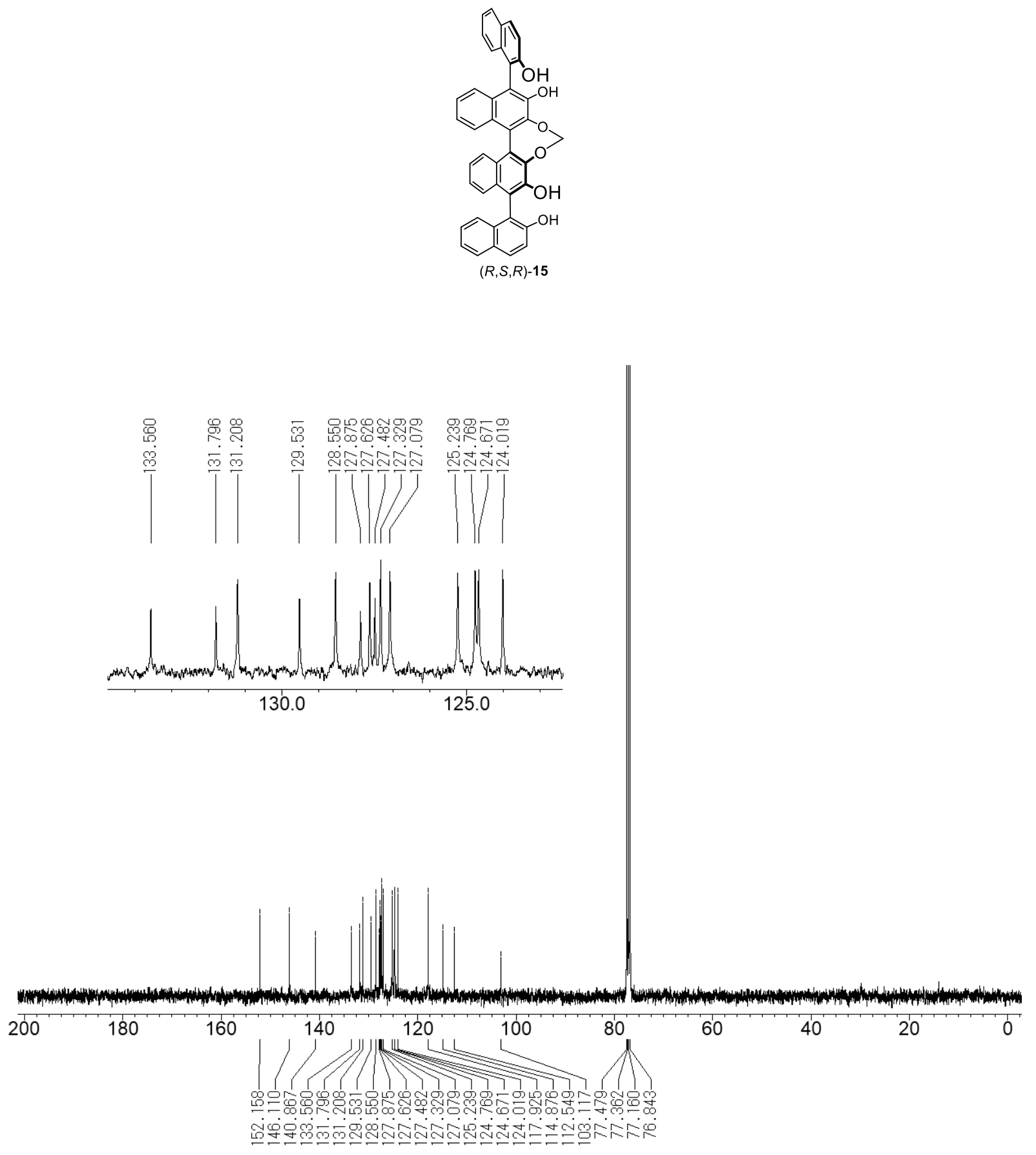

${ }^{13} \mathrm{C}$ NMR of $(R, S, R)-\mathbf{1 5}\left(100 \mathrm{MHz}, \mathrm{CDCl}_{3}\right)$ 


\section{Coordinates of optimized structures}

Coordinates of $(R)-\mathbf{2}$ in Figure 2.

\begin{tabular}{|c|c|c|c|}
\hline $\mathrm{C}$ & 7.750417 & 0.525002 & 0.461460 \\
\hline $\mathrm{C}$ & 7.109919 & -0.354240 & -0.461180 \\
\hline $\mathrm{C}$ & 5.689929 & -0.434136 & -0.401587 \\
\hline $\mathrm{C}$ & 4.973953 & 0.336101 & 0.541145 \\
\hline $\mathrm{C}$ & 5.628336 & 1.177946 & 1.415093 \\
\hline $\mathrm{C}$ & 7.035461 & 1.274103 & 1.376819 \\
\hline $\mathrm{C}$ & 7.784561 & -1.147491 & -1.427568 \\
\hline $\mathrm{C}$ & 7.064013 & -1.963355 & -2.275411 \\
\hline $\mathrm{C}$ & 5.651750 & -2.042011 & -2.217241 \\
\hline C & 4.975447 & -1.282597 & -1.286609 \\
\hline $\mathrm{O}$ & 3.593480 & -1.369035 & -1.239568 \\
\hline $\mathrm{C}$ & -3.548031 & -1.859787 & 2.372341 \\
\hline $\mathrm{C}$ & -2.877513 & -2.639954 & 3.289898 \\
\hline $\mathrm{C}$ & -1.466435 & -2.573338 & 3.339158 \\
\hline $\mathrm{C}$ & -0.740861 & -1.754842 & 2.496710 \\
\hline $\mathrm{C}$ & -1.477325 & 0.707244 & -0.286535 \\
\hline $\mathrm{C}$ & -2.895859 & 0.617051 & -0.314399 \\
\hline $\mathrm{C}$ & -3.544225 & -0.222163 & 0.562197 \\
\hline $\mathrm{C}$ & -2.829566 & -1.005627 & 1.494900 \\
\hline $\mathrm{C}$ & -1.408165 & -0.940867 & 1.539894 \\
\hline $\mathrm{C}$ & -0.745714 & -0.054888 & 0.622403 \\
\hline $\mathrm{C}$ & 0.740747 & 1.755169 & 2.496479 \\
\hline $\mathrm{C}$ & 1.466308 & 2.573780 & 3.338826 \\
\hline $\mathrm{C}$ & 2.877388 & 2.640392 & 3.289573 \\
\hline $\mathrm{C}$ & 3.547919 & 1.860105 & 2.372127 \\
\hline $\mathrm{O}$ & 4.931270 & 1.935304 & 2.333742 \\
\hline $\mathrm{C}$ & 2.895784 & -0.617071 & -0.314307 \\
\hline $\mathrm{C}$ & 1.477257 & -0.707258 & -0.286455 \\
\hline $\mathrm{C}$ & 0.745629 & 0.054957 & 0.622403 \\
\hline $\mathrm{C}$ & 1.408064 & 0.941064 & 1.539783 \\
\hline $\mathrm{C}$ & 2.829465 & 1.005829 & 1.494791 \\
\hline $\mathrm{C}$ & 3.544137 & 0.222245 & 0.562198 \\
\hline $\mathrm{C}$ & -4.974041 & -0.336033 & 0.541135 \\
\hline $\mathrm{C}$ & -5.690007 & 0.434069 & -0.401716 \\
\hline
\end{tabular}




\begin{tabular}{|c|c|c|c|}
\hline $\mathrm{C}$ & -7.109996 & 0.354158 & -0.461320 \\
\hline $\mathrm{C}$ & -7.750504 & -0.524958 & 0.461432 \\
\hline $\mathrm{C}$ & -7.035560 & -1.273930 & 1.376905 \\
\hline $\mathrm{C}$ & -5.628435 & -1.177762 & 1.415185 \\
\hline $\mathrm{C}$ & -4.975514 & 1.282403 & -1.286849 \\
\hline $\mathrm{C}$ & -5.651806 & 2.041683 & -2.217599 \\
\hline $\mathrm{C}$ & -7.064068 & 1.963016 & -2.275777 \\
\hline $\mathrm{C}$ & -7.784626 & 1.147269 & -1.427832 \\
\hline $\mathrm{O}$ & -3.593549 & 1.368863 & -1.239788 \\
\hline $\mathrm{O}$ & -4.931381 & -1.934997 & 2.333945 \\
\hline $\mathrm{H}$ & 8.833300 & 0.605012 & 0.437986 \\
\hline $\mathrm{H}$ & 7.536685 & 1.939996 & 2.071289 \\
\hline $\mathrm{H}$ & 8.867652 & -1.103801 & -1.492006 \\
\hline $\mathrm{H}$ & 7.585702 & -2.566109 & -3.012736 \\
\hline $\mathrm{H}$ & 5.097318 & -2.684948 & -2.891637 \\
\hline $\mathrm{H}$ & -3.436075 & -3.288206 & 3.955571 \\
\hline $\mathrm{H}$ & -0.943453 & -3.187699 & 4.066101 \\
\hline $\mathrm{H}$ & 0.340508 & -1.722360 & 2.562368 \\
\hline $\mathrm{H}$ & -0.340624 & 1.722693 & 2.562128 \\
\hline $\mathrm{H}$ & 0.943316 & 3.188235 & 4.065682 \\
\hline $\mathrm{H}$ & 3.435941 & 3.288732 & 3.955168 \\
\hline $\mathrm{H}$ & -8.833387 & -0.604977 & 0.437951 \\
\hline $\mathrm{H}$ & -7.536792 & -1.939731 & 2.071457 \\
\hline $\mathrm{H}$ & -5.097367 & 2.684528 & -2.892076 \\
\hline $\mathrm{H}$ & -7.585749 & 2.565664 & -3.013195 \\
\hline $\mathrm{H}$ & -8.867716 & 1.103564 & -1.492281 \\
\hline $\mathrm{O}$ & -0.821627 & 1.488544 & -1.195770 \\
\hline $\mathrm{O}$ & 0.821607 & -1.488668 & -1.195625 \\
\hline $\mathrm{C}$ & -0.935727 & 2.915658 & -1.019756 \\
\hline $\mathrm{H}$ & -1.989907 & 3.213650 & -1.061057 \\
\hline $\mathrm{H}$ & -0.535589 & 3.187972 & -0.033474 \\
\hline $\mathrm{C}$ & 0.935940 & -2.915750 & -1.019579 \\
\hline $\mathrm{H}$ & 1.990167 & -3.213569 & -1.061007 \\
\hline $\mathrm{H}$ & 0.535976 & -3.188106 & -0.033237 \\
\hline $\mathrm{C}$ & -0.139034 & 3.579588 & -2.131574 \\
\hline $\mathrm{H}$ & 0.895436 & 3.218112 & -2.085504 \\
\hline $\mathrm{H}$ & -0.544535 & 3.250228 & -3.096242 \\
\hline
\end{tabular}




$\begin{array}{llll}\mathrm{C} & -0.167016 & 5.110411 & -2.043130 \\ \mathrm{H} & -1.207833 & 5.460141 & -2.075025 \\ \mathrm{H} & 0.231018 & 5.427722 & -1.069930 \\ \mathrm{C} & 0.630958 & 5.784603 & -3.163239 \\ \mathrm{H} & 0.595576 & 6.875327 & -3.077340 \\ \mathrm{H} & 1.683516 & 5.481777 & -3.136008 \\ \mathrm{H} & 0.235400 & 5.514446 & -4.148676 \\ \mathrm{C} & 0.139215 & -3.579839 & -2.131280 \\ \mathrm{H} & -0.895334 & -3.218613 & -2.085018 \\ \mathrm{H} & 0.544466 & -3.250358 & -3.096012 \\ \mathrm{C} & 0.167580 & -5.110656 & -2.042883 \\ \mathrm{H} & 1.208477 & -5.460133 & -2.074938 \\ \mathrm{H} & -0.230239 & -5.428092 & -1.069636 \\ \mathrm{C} & -0.630391 & -5.785008 & -3.162899 \\ \mathrm{H} & -0.594728 & -6.875725 & -3.077040 \\ \mathrm{H} & -1.683019 & -5.482441 & -3.135506 \\ \mathrm{H} & -0.235041 & -5.514722 & -4.148383\end{array}$

Coordinates of $(R)-3$ in Figure 2.

$\begin{array}{lrrr}\mathrm{C} & 7.760002 & -0.002424 & -0.233481 \\ \mathrm{C} & 7.035721 & -1.100475 & 0.319105 \\ \mathrm{C} & 5.616038 & -1.000917 & 0.331003 \\ \mathrm{C} & 4.981662 & 0.151212 & -0.185367 \\ \mathrm{C} & 5.715499 & 1.188207 & -0.720143 \\ \mathrm{C} & 7.124276 & 1.113692 & -0.743420 \\ \mathrm{C} & 7.628245 & -2.277072 & 0.850586 \\ \mathrm{C} & 6.830903 & -3.283202 & 1.356404 \\ \mathrm{C} & 5.418662 & -3.186329 & 1.363974 \\ \mathrm{C} & 4.821419 & -2.053799 & 0.853612 \\ \mathrm{O} & -0.708519 & -1.804665 & -0.942322 \\ \mathrm{C} & -0.000005 & -2.589195 & -0.000203 \\ \mathrm{O} & 0.708442 & -1.804741 & 0.942009 \\ \mathrm{O} & 3.437806 & -1.976250 & 0.858736 \\ \mathrm{C} & -3.710602 & 2.378770 & 1.244378 \\ \mathrm{C} & -3.123681 & 3.479335 & 1.829048 \\ \mathrm{C} & -1.713856 & 3.559975 & 1.861958 \\ \mathrm{C} & -0.909483 & 2.585700 & 1.306622\end{array}$




\begin{tabular}{|c|c|c|c|}
\hline $\mathrm{C}$ & -1.417352 & -0.755154 & -0.399065 \\
\hline $\mathrm{C}$ & -2.826509 & -0.841882 & -0.371261 \\
\hline $\mathrm{C}$ & -3.550582 & 0.211408 & 0.142261 \\
\hline $\mathrm{C}$ & -2.909147 & 1.352394 & 0.673768 \\
\hline $\mathrm{C}$ & -1.486543 & 1.453009 & 0.669139 \\
\hline $\mathrm{C}$ & -0.741605 & 0.378057 & 0.059462 \\
\hline $\mathrm{C}$ & 0.909562 & 2.585781 & -1.306608 \\
\hline $\mathrm{C}$ & 1.713971 & 3.560080 & -1.861849 \\
\hline $\mathrm{C}$ & 3.123793 & 3.479390 & -1.828938 \\
\hline $\mathrm{C}$ & 3.710675 & 2.378746 & -1.244378 \\
\hline $\mathrm{O}$ & 5.094315 & 2.303386 & -1.240340 \\
\hline $\mathrm{C}$ & 2.826471 & -0.842003 & 0.371014 \\
\hline $\mathrm{C}$ & 1.417321 & -0.755220 & 0.398823 \\
\hline $\mathrm{C}$ & 0.741607 & 0.378040 & -0.059628 \\
\hline $\mathrm{C}$ & 1.486581 & 1.453013 & -0.669223 \\
\hline $\mathrm{C}$ & 2.909182 & 1.352345 & -0.673863 \\
\hline $\mathrm{C}$ & 3.550576 & 0.211294 & -0.142452 \\
\hline $\mathrm{C}$ & -4.981665 & 0.151325 & 0.185276 \\
\hline $\mathrm{C}$ & -5.616072 & -1.000838 & -0.330982 \\
\hline $\mathrm{C}$ & -7.035747 & -1.100464 & -0.318849 \\
\hline $\mathrm{C}$ & -7.759992 & -0.002435 & 0.233831 \\
\hline $\mathrm{C}$ & -7.124234 & 1.113710 & 0.743666 \\
\hline $\mathrm{C}$ & -5.715464 & 1.188281 & 0.720177 \\
\hline $\mathrm{C}$ & -4.821487 & -2.053710 & -0.853664 \\
\hline $\mathrm{C}$ & -5.418759 & -3.186303 & -1.363853 \\
\hline $\mathrm{C}$ & -6.830994 & -3.283236 & -1.356066 \\
\hline $\mathrm{C}$ & -7.628303 & -2.277110 & -0.850187 \\
\hline $\mathrm{O}$ & -3.437881 & -1.976067 & -0.859086 \\
\hline $\mathrm{O}$ & -5.094247 & 2.303481 & 1.240293 \\
\hline $\mathrm{H}$ & 8.844728 & -0.052617 & -0.253900 \\
\hline $\mathrm{H}$ & 7.688852 & 1.938609 & -1.164824 \\
\hline $\mathrm{H}$ & 8.709614 & -2.375679 & 0.853490 \\
\hline $\mathrm{H}$ & 7.290034 & -4.179987 & 1.761395 \\
\hline $\mathrm{H}$ & 4.802887 & -3.984856 & 1.762278 \\
\hline $\mathrm{H}$ & -0.675685 & -3.207614 & 0.599961 \\
\hline $\mathrm{H}$ & 0.675693 & -3.207558 & -0.600413 \\
\hline $\mathrm{H}$ & -3.745317 & 4.252373 & 2.266357 \\
\hline
\end{tabular}




$\begin{array}{lrrr}\mathrm{H} & -1.253628 & 4.415392 & 2.347261 \\ \mathrm{H} & 0.167341 & 2.677701 & 1.364224 \\ \mathrm{H} & -0.167258 & 2.677820 & -1.364210 \\ \mathrm{H} & 1.253775 & 4.415559 & -2.347075 \\ \mathrm{H} & 3.745455 & 4.252452 & -2.266168 \\ \mathrm{H} & -8.844712 & -0.052677 & 0.254421 \\ \mathrm{H} & -7.688780 & 1.938602 & 1.165160 \\ \mathrm{H} & -4.803010 & -3.984830 & -1.762197 \\ \mathrm{H} & -7.290148 & -4.180064 & -1.760935 \\ \mathrm{H} & -8.709669 & -2.375759 & -0.852930\end{array}$

Coordinates of $(R, R)-4$ in Figure 2.

$\begin{array}{llll}\mathrm{C} & 7.352441 & -3.067731 & -2.657947 \\ \mathrm{C} & 5.958613 & -3.252969 & -2.486098 \\ \mathrm{C} & 5.176814 & -2.256449 & -1.948155 \\ \mathrm{C} & 5.743479 & -1.010861 & -1.547852 \\ \mathrm{C} & 7.156808 & -0.829065 & -1.722810 \\ \mathrm{C} & 7.933267 & -1.879452 & -2.282610 \\ \mathrm{C} & 4.959213 & 0.039395 & -0.982091 \\ \mathrm{C} & 5.579628 & 1.228738 & -0.606273 \\ \mathrm{C} & 6.976219 & 1.406213 & -0.780492 \\ \mathrm{C} & 7.737886 & 0.398746 & -1.325034 \\ \mathrm{C} & 3.485871 & -0.116286 & -0.779548 \\ \mathrm{C} & 2.572478 & 0.098859 & -1.868777 \\ \mathrm{C} & 1.181281 & -0.066121 & -1.616284 \\ \mathrm{C} & 0.730873 & -0.415061 & -0.324018 \\ \mathrm{C} & 1.615736 & -0.606391 & 0.711643 \\ \mathrm{C} & 3.010774 & -0.455293 & 0.485250 \\ \mathrm{C} & 2.965741 & 0.460101 & -3.186354 \\ \mathrm{C} & 2.015202 & 0.634610 & -4.171643 \\ \mathrm{C} & 0.633816 & 0.468149 & -3.920186 \\ \mathrm{C} & 0.227271 & 0.122547 & -2.649594 \\ \mathrm{O} & -4.973155 & 1.258503 & 1.140245 \\ \mathrm{C} & -5.183959 & 4.745949 & 2.544464 \\ \mathrm{O} & -3.839012 & -0.383253 & -1.963868 \\ \mathrm{O} & -1.130647 & -0.034266 & -2.414796 \\ \mathrm{C} & -7.613751 & -3.633890 & 0.391207\end{array}$




\begin{tabular}{|c|c|c|c|}
\hline $\mathrm{C}$ & -6.904705 & -4.733006 & -0.031894 \\
\hline $\mathrm{C}$ & -5.513128 & -4.622264 & -0.272357 \\
\hline $\mathrm{C}$ & -4.859930 & -3.425680 & -0.087675 \\
\hline $\mathrm{C}$ & -5.655125 & 0.081982 & 0.982023 \\
\hline $\mathrm{C}$ & -7.048491 & -0.034054 & 1.221374 \\
\hline $\mathrm{C}$ & -7.682177 & -1.239632 & 1.030198 \\
\hline $\mathrm{C}$ & -6.970746 & -2.382659 & 0.592787 \\
\hline $\mathrm{C}$ & -5.561336 & -2.266106 & 0.350071 \\
\hline $\mathrm{C}$ & -4.908740 & -1.012638 & 0.555209 \\
\hline $\mathrm{C}$ & -2.916595 & -1.430839 & 2.749611 \\
\hline $\mathrm{C}$ & -1.966480 & -1.602709 & 3.735853 \\
\hline $\mathrm{C}$ & -0.585086 & -1.436526 & 3.484443 \\
\hline $\mathrm{C}$ & -0.178248 & -1.092957 & 2.213276 \\
\hline $\mathrm{O}$ & 4.770816 & 2.191848 & -0.081121 \\
\hline $\mathrm{O}$ & 3.885262 & -0.582079 & 1.531051 \\
\hline $\mathrm{C}$ & 5.339396 & 3.405304 & 0.407011 \\
\hline $\mathrm{O}$ & 1.179449 & -0.934549 & 1.979059 \\
\hline $\mathrm{C}$ & -1.566478 & -0.364626 & -1.148124 \\
\hline $\mathrm{C}$ & -2.961802 & -0.517823 & -0.921832 \\
\hline $\mathrm{C}$ & -3.435744 & -0.863712 & 0.340808 \\
\hline $\mathrm{C}$ & -2.522786 & -1.074603 & 1.431083 \\
\hline $\mathrm{C}$ & -1.131976 & -0.907385 & 1.179247 \\
\hline $\mathrm{C}$ & -0.681688 & -0.556227 & -0.112581 \\
\hline $\mathrm{H}$ & 7.955736 & -3.863392 & -3.084633 \\
\hline $\mathrm{H}$ & 5.501993 & -4.192401 & -2.784520 \\
\hline $\mathrm{H}$ & 4.110167 & -2.409309 & -1.825853 \\
\hline $\mathrm{H}$ & 9.001990 & -1.723573 & -2.407604 \\
\hline $\mathrm{H}$ & 7.448219 & 2.335506 & -0.486359 \\
\hline $\mathrm{H}$ & 8.807389 & 0.541460 & -1.455945 \\
\hline $\mathrm{H}$ & 4.017769 & 0.595737 & -3.408401 \\
\hline $\mathrm{H}$ & 2.330253 & 0.908948 & -5.174138 \\
\hline $\mathrm{H}$ & -0.103594 & 0.606011 & -4.702983 \\
\hline $\mathrm{H}$ & -5.611540 & 4.494683 & 3.524521 \\
\hline $\mathrm{H}$ & -6.019684 & 5.111763 & 1.932524 \\
\hline $\mathrm{H}$ & -8.682314 & -3.704165 & 0.578960 \\
\hline $\mathrm{H}$ & -7.405832 & -5.684404 & -0.183339 \\
\hline $\mathrm{H}$ & -4.956623 & -5.492508 & -0.608371 \\
\hline
\end{tabular}




\begin{tabular}{|c|c|c|c|}
\hline $\mathrm{H}$ & -3.795038 & -3.351858 & -0.278168 \\
\hline $\mathrm{H}$ & -7.618994 & 0.825321 & 1.551611 \\
\hline$\pi$ & -8.749928 & -1.323176 & 1.215252 \\
\hline $\mathrm{H}$ & -3.968726 & -1.567895 & 2.970782 \\
\hline $\mathrm{H}$ & -2.281699 & -1.875406 & 4.738787 \\
\hline $\mathrm{H}$ & 0.152234 & -1.573606 & 4.267479 \\
\hline $\mathrm{H}$ & 6.093045 & 3.186396 & 1.177112 \\
\hline$H$ & 5.839267 & 3.946640 & -0.409884 \\
\hline C & -6.511880 & 2.225975 & -5.203527 \\
\hline $\mathrm{C}$ & 4.206970 & 4.240944 & 0.987150 \\
\hline $\mathrm{H}$ & 3.458472 & 4.408279 & 0.203062 \\
\hline$\Pi$ & 3.712282 & 3.658851 & 1.773859 \\
\hline $\mathrm{C}$ & 4.692401 & 5.583070 & 1.547835 \\
\hline $\mathrm{H}$ & 5.448916 & 5.403640 & 2.323773 \\
\hline$\Pi$ & 5.198304 & 6.150445 & 0.754788 \\
\hline $\mathrm{C}$ & 3.556060 & 6.427922 & 2.131531 \\
\hline $\mathrm{H}$ & 3.928298 & 7.379078 & 2.525042 \\
\hline $\mathrm{H}$ & 2.800705 & 6.654096 & 1.370852 \\
\hline $\mathrm{H}$ & 3.052852 & 5.901855 & 2.950228 \\
\hline $\mathrm{C}$ & 4.119490 & -1.924434 & 2.000495 \\
\hline $\mathrm{H}$ & 3.174440 & -2.374702 & 2.327501 \\
\hline $\mathrm{H}$ & 4.525035 & -2.527817 & 1.176823 \\
\hline $\mathrm{C}$ & -5.628722 & 2.376488 & 1.738238 \\
\hline $\mathrm{H}$ & -6.458552 & 2.717113 & 1.101648 \\
\hline $\mathrm{H}$ & -6.049305 & 2.089311 & 2.712649 \\
\hline C & 5.109275 & -1.843735 & 3.151947 \\
\hline $\mathrm{H}$ & 6.019806 & -1.346038 & 2.796308 \\
\hline $\mathrm{H}$ & 4.683869 & -1.202371 & 3.933981 \\
\hline $\mathrm{C}$ & 5.454079 & -3.221256 & 3.731039 \\
\hline $\mathrm{H}$ & 4.534022 & -3.716744 & 4.069338 \\
\hline $\mathrm{H}$ & 5.868469 & -3.857543 & 2.937642 \\
\hline $\mathrm{C}$ & -4.595833 & 3.482326 & 1.904829 \\
\hline $\mathrm{C}$ & 6.448272 & -3.145706 & 4.893692 \\
\hline $\mathrm{H}$ & 6.677503 & -4.140818 & 5.288112 \\
\hline $\mathrm{H}$ & 7.391770 & -2.686851 & 4.577764 \\
\hline $\mathrm{H}$ & 6.048539 & -2.544324 & 5.717870 \\
\hline $\mathrm{C}$ & -4.149251 & 5.862603 & 2.712238 \\
\hline
\end{tabular}




$\begin{array}{llll}\text { C } & -4.067052 & 0.963533 & -2.425899 \\ \text { H } & -4.409455 & 1.575916 & -1.581498 \\ \text { H } & -3.130183 & 1.388769 & -2.805963 \\ \text { C } & -5.119033 & 0.900897 & -3.522086 \\ \text { H } & -4.754482 & 0.241049 & -4.319175 \\ \text { H } & -6.022847 & 0.431611 & -3.114662 \\ \text { C } & -5.454683 & 2.282542 & -4.096696 \\ \text { H } & -5.806923 & 2.938705 & -3.289102 \\ \text { H } & -4.540286 & 2.747927 & -4.488848 \\ \text { H } & -6.733568 & 3.223727 & -5.595763 \\ \text { H } & -6.174125 & 1.605804 & -6.041235 \\ \text { H } & -7.449540 & 1.796871 & -4.833166 \\ \text { H } & -3.769095 & 3.099098 & 2.514825 \\ \text { H } & -4.173892 & 3.720941 & 0.920684 \\ \text { H } & -4.593632 & 6.751348 & 3.171155 \\ \text { H } & -3.317837 & 5.538866 & 3.347909 \\ \text { H } & -3.728935 & 6.161020 & 1.745489\end{array}$

Coordinates of $(R, R)-5$ in Figure 2.

$\begin{array}{lrrr}\text { C } & -7.632482 & 1.438532 & 2.662616 \\ \mathrm{C} & -6.232006 & 1.584690 & 2.797033 \\ \mathrm{C} & -5.374621 & 1.010625 & 1.885294 \\ \mathrm{C} & -5.866874 & 0.255750 & 0.784046 \\ \mathrm{C} & -7.287236 & 0.071094 & 0.678112 \\ \mathrm{C} & -8.143492 & 0.692442 & 1.626860 \\ \mathrm{C} & -5.004499 & -0.364076 & -0.185195 \\ \mathrm{C} & -5.577825 & -1.223038 & -1.114758 \\ \mathrm{C} & -6.972215 & -1.408995 & -1.219011 \\ \mathrm{C} & -7.811501 & -0.752022 & -0.352997 \\ \mathrm{C} & -3.527260 & -0.178837 & -0.221775 \\ \mathrm{C} & -2.891828 & 1.115183 & -0.280851 \\ \mathrm{C} & -1.466161 & 1.153126 & -0.276176 \\ \mathrm{C} & -0.715115 & -0.044181 & -0.258420 \\ \mathrm{C} & -1.333649 & -1.273925 & -0.282701 \\ \mathrm{C} & -2.745084 & -1.333037 & -0.273382 \\ \mathrm{C} & -3.578608 & 2.356033 & -0.387478 \\ \mathrm{C} & -2.874592 & 3.541659 & -0.443332\end{array}$




\begin{tabular}{|c|c|c|c|}
\hline $\mathrm{C}$ & -1.463112 & 3.579728 & -0.403306 \\
\hline $\mathrm{C}$ & -0.771228 & 2.391223 & -0.327854 \\
\hline $\mathrm{O}$ & 4.768385 & 1.886389 & -2.022736 \\
\hline $\mathrm{C}$ & 3.989032 & 2.930674 & -1.475026 \\
\hline $\mathrm{O}$ & 3.348187 & 2.571170 & -0.263391 \\
\hline $\mathrm{O}$ & 0.613810 & 2.444825 & -0.319415 \\
\hline $\mathrm{C}$ & 8.143561 & -0.692063 & 1.626946 \\
\hline $\mathrm{C}$ & 7.632603 & -1.437968 & 2.662860 \\
\hline $\mathrm{C}$ & 6.232136 & -1.584124 & 2.797353 \\
\hline $\mathrm{C}$ & 5.374710 & -1.010257 & 1.885526 \\
\hline $\mathrm{C}$ & 5.577778 & 1.222877 & -1.114930 \\
\hline $\mathrm{C}$ & 6.972159 & 1.408860 & -1.219242 \\
\hline $\mathrm{C}$ & 7.811481 & 0.752037 & -0.353153 \\
\hline $\mathrm{C}$ & 7.287260 & -0.070919 & 0.678105 \\
\hline $\mathrm{C}$ & 5.866904 & -0.255602 & 0.784100 \\
\hline $\mathrm{C}$ & 5.004491 & 0.364020 & -0.185247 \\
\hline $\mathrm{C}$ & 3.578589 & -2.356156 & -0.387444 \\
\hline $\mathrm{C}$ & 2.874569 & -3.541782 & -0.443221 \\
\hline $\mathrm{C}$ & 1.463091 & -3.579848 & -0.403133 \\
\hline $\mathrm{C}$ & 0.771215 & -2.391338 & -0.327719 \\
\hline $\mathrm{O}$ & -4.768466 & -1.886674 & -2.022503 \\
\hline $\mathrm{O}$ & -3.348211 & -2.571278 & -0.263123 \\
\hline $\mathrm{C}$ & -3.989099 & -2.930901 & -1.474699 \\
\hline $\mathrm{O}$ & -0.613823 & -2.444940 & -0.319243 \\
\hline $\mathrm{C}$ & 1.333637 & 1.273811 & -0.282811 \\
\hline $\mathrm{C}$ & 2.745071 & 1.332925 & -0.273520 \\
\hline $\mathrm{C}$ & 3.527254 & 0.178734 & -0.221819 \\
\hline $\mathrm{C}$ & 2.891823 & -1.115294 & -0.280828 \\
\hline $\mathrm{C}$ & 1.466154 & -1.153239 & -0.276126 \\
\hline $\mathrm{C}$ & 0.715106 & 0.044068 & -0.258432 \\
\hline $\mathrm{H}$ & -8.297567 & 1.902264 & 3.384867 \\
\hline $\mathrm{H}$ & -5.828013 & 2.149533 & 3.632010 \\
\hline $\mathrm{H}$ & -4.304534 & 1.121636 & 2.011241 \\
\hline $\mathrm{H}$ & -9.216450 & 0.552560 & 1.524292 \\
\hline $\mathrm{H}$ & -7.349453 & -2.065394 & -1.996085 \\
\hline $\mathrm{H}$ & -8.888023 & -0.876988 & -0.429635 \\
\hline $\mathrm{H}$ & -4.659804 & 2.367552 & -0.435211 \\
\hline
\end{tabular}




$\begin{array}{lrrr}\mathrm{H} & -3.417448 & 4.478234 & -0.528038 \\ \mathrm{H} & -0.919814 & 4.516807 & -0.446578 \\ \mathrm{H} & 4.602665 & 3.800535 & -1.214034 \\ \mathrm{H} & 3.261650 & 3.181119 & -2.254818 \\ \mathrm{H} & 9.216513 & -0.552169 & 1.524323 \\ \mathrm{H} & 8.297722 & -1.901540 & 3.385182 \\ \mathrm{H} & 5.828181 & -2.148805 & 3.632457 \\ \mathrm{H} & 4.304633 & -1.121260 & 2.011545 \\ \mathrm{H} & 7.349360 & 2.065163 & -1.996416 \\ \mathrm{H} & 8.887998 & 0.877027 & -0.429828 \\ \mathrm{H} & 4.659779 & -2.367687 & -0.435230 \\ \mathrm{H} & 3.417419 & -4.478362 & -0.527911 \\ \mathrm{H} & 0.919789 & -4.516927 & -0.446344 \\ \mathrm{H} & -3.261745 & -3.181435 & -2.254488 \\ \mathrm{H} & -4.602728 & -3.800732 & -1.213595\end{array}$

Coordinates of $(R)-\mathbf{2}$ in Figure S6.

$\begin{array}{lrrr}\text { C } & -7.772096 & -0.531453 & -0.239868 \\ \text { C } & -7.078525 & 0.531253 & 0.405077 \\ \text { C } & -5.654992 & 0.473153 & 0.422795 \\ \text { C } & -4.974495 & -0.614487 & -0.184601 \\ \text { C } & -5.696306 & -1.634950 & -0.805932 \\ \text { C } & -7.096618 & -1.594886 & -0.834612 \\ \text { C } & -7.699087 & 1.642510 & 1.033673 \\ \text { C } & -6.930060 & 2.622860 & 1.637775 \\ \text { C } & -5.520962 & 2.561704 & 1.654457 \\ \text { C } & -4.893557 & 1.486339 & 1.046220 \\ \text { O } & -3.514916 & 1.439942 & 1.067958 \\ \text { C } & 3.652418 & -2.738310 & 1.376371 \\ \text { C } & 3.038202 & -3.802030 & 2.018434 \\ \text { C } & 1.634225 & -3.852680 & 2.027880 \\ \text { C } & 0.860874 & -2.884187 & 1.402021 \\ \text { C } & 1.451057 & 0.345782 & -0.527779 \\ \text { C } & 2.848434 & 0.386993 & -0.473518 \\ \text { C } & 3.566579 & -0.645380 & 0.140711 \\ \text { C } & 2.885670 & -1.740634 & 0.730733 \\ \text { C } & 1.464019 & -1.805911 & 0.705988\end{array}$




\begin{tabular}{|c|c|c|c|}
\hline $\mathrm{C}$ & 0.732614 & -0.745000 & 0.037180 \\
\hline $\mathrm{C}$ & -0.860854 & -2.884313 & -1.401769 \\
\hline $\mathrm{C}$ & -1.634204 & -3.852864 & -2.027539 \\
\hline $\mathrm{C}$ & -3.038180 & -3.802216 & -2.018095 \\
\hline $\mathrm{C}$ & -3.652399 & -2.738441 & -1.376127 \\
\hline $\mathrm{O}$ & -5.033765 & -2.686107 & -1.400010 \\
\hline $\mathrm{C}$ & -2.848421 & 0.387030 & 0.473484 \\
\hline $\mathrm{C}$ & -1.451045 & 0.345827 & 0.527747 \\
\hline $\mathrm{C}$ & -0.732600 & -0.745006 & -0.037115 \\
\hline $\mathrm{C}$ & -1.464001 & -1.805977 & -0.705829 \\
\hline $\mathrm{C}$ & -2.885653 & -1.740705 & -0.730578 \\
\hline $\mathrm{C}$ & -3.566563 & -0.645400 & -0.140652 \\
\hline $\mathrm{C}$ & 4.974509 & -0.614459 & 0.184659 \\
\hline $\mathrm{C}$ & 5.655004 & 0.473128 & -0.422834 \\
\hline $\mathrm{C}$ & 7.078537 & 0.531234 & -0.405119 \\
\hline $\mathrm{C}$ & 7.772110 & -0.531413 & 0.239922 \\
\hline $\mathrm{C}$ & 7.096635 & -1.594795 & 0.834760 \\
\hline $\mathrm{C}$ & 5.696323 & -1.634866 & 0.806083 \\
\hline $\mathrm{C}$ & 4.893566 & 1.486254 & -1.046351 \\
\hline $\mathrm{C}$ & 5.520969 & 2.561568 & -1.654685 \\
\hline $\mathrm{C}$ & 6.930066 & 2.622729 & -1.638006 \\
\hline $\mathrm{C}$ & 7.699096 & 1.642436 & -1.033813 \\
\hline $\mathrm{O}$ & 3.514927 & 1.439851 & -1.068088 \\
\hline $\mathrm{O}$ & 5.033784 & -2.685970 & 1.400252 \\
\hline $\mathrm{H}$ & -8.857195 & -0.508896 & -0.266365 \\
\hline $\mathrm{H}$ & -7.637775 & -2.397689 & -1.323813 \\
\hline $\mathrm{H}$ & -8.782535 & 1.716928 & 1.037098 \\
\hline $\mathrm{H}$ & -7.418859 & 3.467481 & 2.114124 \\
\hline $\mathrm{H}$ & -4.925814 & 3.335014 & 2.126611 \\
\hline $\mathrm{H}$ & 3.641605 & -4.556598 & 2.509925 \\
\hline $\mathrm{H}$ & 1.142499 & -4.669215 & 2.547893 \\
\hline $\mathrm{H}$ & -0.219303 & -2.951613 & 1.446289 \\
\hline $\mathrm{H}$ & 0.219324 & -2.951741 & -1.446034 \\
\hline $\mathrm{H}$ & -1.142476 & -4.669444 & -2.547481 \\
\hline $\mathrm{H}$ & -3.641583 & -4.556829 & -2.509519 \\
\hline $\mathrm{H}$ & 8.857209 & -0.508851 & 0.266419 \\
\hline $\mathrm{H}$ & 7.637794 & -2.397552 & 1.324033 \\
\hline
\end{tabular}




\begin{tabular}{lrrr}
$\mathrm{H}$ & 4.925819 & 3.334833 & -2.126909 \\
$\mathrm{H}$ & 7.418864 & 3.467308 & -2.114430 \\
$\mathrm{H}$ & 8.782543 & 1.716857 & -1.037245 \\
$\mathrm{O}$ & 0.778376 & 1.353557 & -1.165696 \\
$\mathrm{O}$ & -0.778359 & 1.353658 & 1.165571 \\
$\mathrm{C}$ & 0.740119 & 1.250485 & -2.601023 \\
$\mathrm{H}$ & 1.761682 & 1.277809 & -3.003117 \\
$\mathrm{H}$ & 0.285666 & 0.290680 & -2.884757 \\
$\mathrm{C}$ & -0.740158 & 1.250758 & 2.600910 \\
$\mathrm{H}$ & -1.761735 & 1.278149 & 3.002964 \\
$\mathrm{H}$ & -0.285732 & 0.290980 & 2.884777 \\
$\mathrm{C}$ & -0.080552 & 2.417412 & -3.127625 \\
$\mathrm{H}$ & -1.077089 & 2.377706 & -2.671310 \\
$\mathrm{H}$ & 0.383028 & 3.351408 & -2.786660 \\
$\mathrm{C}$ & -0.199168 & 2.415025 & -4.656500 \\
$\mathrm{H}$ & 0.804677 & 2.440913 & -5.101893 \\
$\mathrm{H}$ & -0.653926 & 1.471292 & -4.986648 \\
$\mathrm{C}$ & -1.023206 & 3.590466 & -5.190700 \\
$\mathrm{H}$ & -1.093110 & 3.565483 & -6.282922 \\
$\mathrm{H}$ & -2.042985 & 3.572229 & -4.790559 \\
$\mathrm{H}$ & -0.574892 & 4.549140 & -4.906850 \\
$\mathrm{C}$ & 0.080514 & 2.417735 & 3.127402 \\
$\mathrm{H}$ & 1.077066 & 2.377959 & 2.671128 \\
$\mathrm{H}$ & -0.383039 & 3.351698 & 2.786309 \\
$\mathrm{H}$ & 0.199075 & 2.415531 & 4.656282 \\
$\mathrm{H}$ & -0.804785 & 2.441487 & 5.101636 \\
$\mathrm{H}$ & 0.653806 & 1.471830 & 4.986559 \\
\hline & 1.023112 & 3.591022 & 5.190369 \\
$\mathrm{H}$ & 2.042905 & 3.572723 & 4.790267 \\
$\mathrm{H}$ & & & \\
$\mathrm{H}$ & & & \\
$\mathrm{H}$ & .574823 & 4.549669 & 4.906389
\end{tabular}

Coordinates of $(R)-\mathbf{3}$ in Figure $\mathrm{S} 6$.
$\begin{array}{lll}\text { C } & -7.768909 & -0.042159\end{array}$
$-0.195215$
$\begin{array}{lll}\text { C } & -7.049199 & 1.076847\end{array}$
0.311388
$\begin{array}{lll}\text { C } & -5.626670 & 0.999619\end{array}$
0.308700
C $\quad-4.973034 \quad-0.161089$
$-0.178716$ 


\begin{tabular}{|c|c|c|c|}
\hline $\mathrm{C}$ & -5.718715 & -1.229840 & -0.676138 \\
\hline $\mathrm{C}$ & -7.119421 & -1.172734 & -0.682499 \\
\hline $\mathrm{C}$ & -7.643343 & 2.262900 & $0.81628 \mathrm{c}$ \\
\hline $\mathrm{C}$ & -6.850023 & 3.297099 & 1.283259 \\
\hline $\mathrm{C}$ & -5.441419 & 3.219495 & 1.275586 \\
\hline $\mathrm{C}$ & -4.839556 & 2.071571 & 0.78704 \\
\hline $\mathrm{O}$ & 0.742374 & 1.867591 & -0.91055 \\
\hline $\mathrm{C}$ & -0.000004 & 2.652595 & -0.00000 \\
\hline $\mathrm{O}$ & -0.742372 & 1.867590 & 0.91055 \\
\hline $\mathrm{O}$ & -3.461773 & 2.013943 & 0.77515 \\
\hline $\mathrm{C}$ & 3.698957 & -2.406478 & 1.18436 \\
\hline $\mathrm{C}$ & 3.112726 & -3.521355 & 1.760178 \\
\hline $\mathrm{C}$ & 1.710483 & -3.582451 & 1.815006 \\
\hline $\mathrm{C}$ & 0.912269 & -2.578734 & 1.283033 \\
\hline $\mathrm{C}$ & 1.432985 & 0.815835 & -0.344540 \\
\hline $\mathrm{C}$ & 2.824862 & 0.881122 & -0.31735 \\
\hline $\mathrm{C}$ & 3.564721 & -0.211338 & 0.150447 \\
\hline $\mathrm{C}$ & 2.905684 & -1.366356 & 0.643472 \\
\hline $\mathrm{C}$ & 1.484386 & -1.451339 & 0.643481 \\
\hline $\mathrm{C}$ & 0.725852 & -0.349636 & 0.067937 \\
\hline $\mathrm{C}$ & -0.912269 & -2.578736 & -1.283032 \\
\hline $\mathrm{C}$ & -1.710483 & -3.582452 & -1.815005 \\
\hline $\mathrm{C}$ & -3.112726 & -3.521356 & -1.760177 \\
\hline $\mathrm{C}$ & -3.698957 & -2.406479 & -1.184360 \\
\hline $\mathrm{O}$ & -5.080845 & -2.344178 & -1.171755 \\
\hline $\mathrm{C}$ & -2.824862 & 0.881122 & 0.317353 \\
\hline $\mathrm{C}$ & -1.432986 & 0.815833 & 0.344539 \\
\hline $\mathrm{C}$ & -0.725852 & -0.349637 & -0.067937 \\
\hline $\mathrm{C}$ & -1.484386 & -1.451341 & -0.643481 \\
\hline $\mathrm{C}$ & -2.905683 & -1.366357 & -0.643472 \\
\hline $\mathrm{C}$ & -3.564721 & -0.211337 & -0.150448 \\
\hline $\mathrm{C}$ & 4.973035 & -0.161089 & 0.178715 \\
\hline $\mathrm{C}$ & 5.626670 & 0.999618 & -0.308700 \\
\hline $\mathrm{C}$ & 7.049200 & 1.076847 & -0.311386 \\
\hline $\mathrm{C}$ & 7.768909 & -0.042159 & 0.195216 \\
\hline $\mathrm{C}$ & 7.119421 & -1.172735 & 0.6825 \\
\hline $\mathrm{C}$ & 5.718715 & -1.229840 & 0.67613 \\
\hline
\end{tabular}




$\begin{array}{lrrr}\mathrm{C} & 4.839556 & 2.071571 & -0.787048 \\ \mathrm{C} & 5.441420 & 3.219495 & -1.275585 \\ \mathrm{C} & 6.850023 & 3.297099 & -1.283256 \\ \mathrm{C} & 7.643343 & 2.262900 & -0.816287 \\ \mathrm{O} & 3.461773 & 2.013942 & -0.775155 \\ \mathrm{O} & 5.080845 & -2.344178 & 1.171753 \\ \mathrm{H} & -8.853998 & -0.007067 & -0.203094 \\ \mathrm{H} & -7.680134 & -2.015612 & -1.072183 \\ \mathrm{H} & -8.725505 & 2.352268 & 0.832798 \\ \mathrm{H} & -7.318203 & 4.198572 & 1.666993 \\ \mathrm{H} & -4.827257 & 4.034631 & 1.640935 \\ \mathrm{H} & 0.651795 & 3.272291 & 0.625557 \\ \mathrm{H} & -0.651810 & 3.272278 & -0.625571 \\ \mathrm{H} & 3.736564 & -4.305483 & 2.173794 \\ \mathrm{H} & 1.240239 & -4.434858 & 2.295543 \\ \mathrm{H} & -0.164375 & -2.657186 & 1.363756 \\ \mathrm{H} & 0.164375 & -2.657188 & -1.363755 \\ \mathrm{H} & -1.240240 & -4.434861 & -2.295542 \\ \mathrm{H} & -3.736565 & -4.305484 & -2.173793 \\ \mathrm{H} & 8.853998 & -0.007067 & 0.203096 \\ \mathrm{H} & 7.680134 & -2.015612 & 1.072183 \\ \mathrm{H} & 4.827258 & 4.034632 & -1.640933 \\ \mathrm{H} & 7.318204 & 4.198572 & -1.666990 \\ & 8.725505 & 2.352269 & -0.832795\end{array}$

Coordinates of $(R, R)-4$ in Figure S6.

$\begin{array}{llll}\mathrm{C} & 7.264558 & -4.336891 & 0.821428 \\ \mathrm{C} & 5.888357 & -4.250005 & 1.133909 \\ \mathrm{C} & 5.129301 & -3.192854 & 0.677913 \\ \mathrm{C} & 5.697315 & -2.163210 & -0.124033 \\ \mathrm{C} & 7.106128 & -2.234376 & -0.401339 \\ \mathrm{C} & 7.856665 & -3.343322 & 0.075684 \\ \mathrm{C} & 4.929960 & -1.050122 & -0.612861 \\ \mathrm{C} & 5.592810 & -0.033956 & -1.315753 \\ \mathrm{C} & 6.981332 & -0.102013 & -1.567197 \\ \mathrm{C} & 7.715590 & -1.183304 & -1.127179 \\ \mathrm{C} & 3.473403 & -0.930756 & -0.373900\end{array}$




\begin{tabular}{|c|c|c|c|}
\hline $\mathrm{C}$ & 2.533951 & -1.856039 & -0.951864 \\
\hline $\mathrm{C}$ & 1.145356 & -1.676822 & -0.697127 \\
\hline $\mathrm{C}$ & 0.689814 & -0.587557 & 0.097179 \\
\hline $\mathrm{C}$ & 1.622028 & 0.319552 & 0.637580 \\
\hline $\mathrm{C}$ & 2.990398 & 0.156573 & 0.406467 \\
\hline $\mathrm{C}$ & 2.887410 & -2.929958 & -1.810072 \\
\hline $\mathrm{C}$ & 1.916642 & -3.774089 & -2.34108 \\
\hline $\mathrm{C}$ & 0.551950 & -3.601894 & -2.06943 \\
\hline $\mathrm{C}$ & 0.177213 & -2.540583 & -1.248844 \\
\hline $\mathrm{O}$ & -4.804249 & 2.065847 & -0.24760 \\
\hline $\mathrm{C}$ & -4.764627 & 5.373344 & -2.04237 \\
\hline $\mathrm{O}$ & -3.849103 & -2.087085 & -0.522314 \\
\hline $\mathrm{O}$ & -1.164611 & -2.371806 & -1.007731 \\
\hline $\mathrm{C}$ & -7.865630 & -1.671662 & 2.60485 \\
\hline $\mathrm{C}$ & -7.263732 & -2.773617 & 3.16646 \\
\hline $\mathrm{C}$ & -5.866611 & -2.949930 & 3.034717 \\
\hline $\mathrm{C}$ & -5.104777 & -2.038095 & 2.337237 \\
\hline $\mathrm{C}$ & -5.577447 & 1.195423 & 0.473135 \\
\hline $\mathrm{C}$ & -6.968472 & 1.380180 & 0.644704 \\
\hline $\mathrm{C}$ & -7.712150 & 0.444706 & 1.331520 \\
\hline $\mathrm{C}$ & -7.107020 & -0.708404 & 1.885403 \\
\hline $\mathrm{C}$ & -5.692273 & -0.896625 & 1.726072 \\
\hline $\mathrm{C}$ & -4.919404 & 0.073896 & 1.000059 \\
\hline $\mathrm{C}$ & -2.876324 & 1.923845 & 2.211176 \\
\hline $\mathrm{C}$ & -1.908025 & 2.773113 & 2.738801 \\
\hline $\mathrm{C}$ & -0.542758 & 2.599061 & 2.471006 \\
\hline $\mathrm{C}$ & -0.166722 & 1.532117 & 1.659488 \\
\hline $\mathrm{O}$ & 4.814672 & 0.978034 & -1.793854 \\
\hline $\mathrm{O}$ & 3.866178 & 1.071548 & 0.936905 \\
\hline $\mathrm{C}$ & 5.418814 & 2.224499 & -2.134951 \\
\hline $\mathrm{O}$ & 1.179406 & 1.362548 & 1.417838 \\
\hline $\mathrm{C}$ & -1.607480 & -1.332873 & -0.221928 \\
\hline $\mathrm{C}$ & -2.976980 & -1.170916 & 0.008991 \\
\hline $\mathrm{C}$ & -3.460032 & -0.086558 & 0.790246 \\
\hline $\mathrm{C}$ & -2.520152 & 0.843128 & 1.365438 \\
\hline $\mathrm{C}$ & -1.131713 & 0.663597 & 1.114109 \\
\hline $\mathrm{C}$ & -0.675286 & -0.426866 & 0.32143 \\
\hline
\end{tabular}




\begin{tabular}{|c|c|c|c|}
\hline $\mathrm{H}$ & 7.851177 & -5.177263 & 1.180793 \\
\hline $\mathrm{H}$ & 5.425461 & -5.019233 & 1.745588 \\
\hline$\pi$ & 4.077895 & -3.135484 & 0.936133 \\
\hline $\mathrm{H}$ & 8.917791 & -3.386909 & -0.157381 \\
\hline $\mathrm{H}$ & 7.466009 & 0.682768 & -2.136141 \\
\hline $\mathrm{H}$ & 8.780571 & -1.236403 & -1.336874 \\
\hline $\mathrm{H}$ & 3.930046 & -3.091478 & -2.055519 \\
\hline $\mathrm{H}$ & 2.223507 & -4.588716 & -2.989855 \\
\hline $\mathrm{H}$ & -0.205027 & -4.258772 & -2.481860 \\
\hline $\mathrm{H}$ & -5.210823 & 5.989294 & -1.249807 \\
\hline $\mathrm{H}$ & -5.570747 & 5.171557 & -2.760774 \\
\hline$\Pi$ & -8.936820 & -1.515228 & 2.706200 \\
\hline $\mathrm{H}$ & -7.853673 & -3.501808 & 3.715348 \\
\hline $\mathrm{H}$ & -5.388148 & -3.811940 & 3.490792 \\
\hline$U$ & -4.034598 & -2.185274 & 2.252191 \\
\hline $\mathrm{H}$ & -7.459859 & 2.246437 & 0.218965 \\
\hline $\mathrm{H}$ & -8.782313 & 0.588959 & 1.453686 \\
\hline $\mathrm{H}$ & -3.919475 & 2.079470 & 2.459363 \\
\hline $\mathrm{H}$ & -2.215955 & 3.591274 & 3.382852 \\
\hline $\mathrm{H}$ & 0.213261 & 3.258598 & 2.881158 \\
\hline $\mathrm{H}$ & 6.134604 & 2.518749 & -1.355183 \\
\hline $\mathrm{H}$ & 5.969916 & 2.134789 & -3.083388 \\
\hline $\mathrm{C}$ & -6.959773 & -3.666664 & -4.029362 \\
\hline $\mathrm{C}$ & 4.304598 & 3.254734 & -2.258005 \\
\hline $\mathrm{H}$ & 3.580581 & 2.900987 & -3.002623 \\
\hline $\mathrm{H}$ & 3.775233 & 3.300153 & -1.299085 \\
\hline C & 4.823369 & 4.643612 & -2.648692 \\
\hline $\mathrm{H}$ & 5.556515 & 4.983198 & -1.904454 \\
\hline $\mathrm{H}$ & 5.365548 & 4.578620 & -3.601975 \\
\hline $\mathrm{C}$ & 3.704644 & 5.682651 & -2.770105 \\
\hline $\mathrm{H}$ & 4.100346 & 6.664871 & -3.047616 \\
\hline $\mathrm{H}$ & 2.974092 & 5.389756 & -3.532370 \\
\hline $\mathrm{H}$ & 3.165731 & 5.794970 & -1.822863 \\
\hline $\mathrm{C}$ & 4.260668 & 0.814884 & 2.298213 \\
\hline $\mathrm{H}$ & 3.379578 & 0.862711 & 2.952911 \\
\hline $\mathrm{H}$ & 4.686681 & -0.194989 & 2.369094 \\
\hline C & -5.378936 & 3.266540 & -0.75750 \\
\hline
\end{tabular}




\begin{tabular}{llll}
$\mathrm{H}$ & -6.183668 & 3.030920 & -1.469666 \\
$\mathrm{H}$ & -5.819326 & 3.854544 & 0.061127 \\
$\mathrm{C}$ & 5.286649 & 1.865299 & 2.693328 \\
$\mathrm{H}$ & 6.133462 & 1.803309 & 1.998664 \\
$\mathrm{H}$ & 4.840010 & 2.858830 & 2.559992 \\
$\mathrm{C}$ & 5.777901 & 1.699175 & 4.136570 \\
$\mathrm{H}$ & 4.921348 & 1.748004 & 4.822469 \\
$\mathrm{H}$ & 6.211309 & 0.697984 & 4.261335 \\
$\mathrm{C}$ & -4.269458 & 4.050980 & -1.444512 \\
$\mathrm{C}$ & 6.812239 & 2.754965 & 4.538254 \\
$\mathrm{H}$ & 7.145931 & 2.613826 & 5.571216 \\
$\mathrm{H}$ & 7.696507 & 2.707430 & 3.893031 \\
$\mathrm{H}$ & 6.398097 & 3.766260 & 4.456966 \\
$\mathrm{C}$ & -3.651770 & 6.164850 & -2.735808 \\
$\mathrm{C}$ & -4.303197 & -1.793016 & -1.856928 \\
$\mathrm{H}$ & -4.722136 & -0.778285 & -1.883737 \\
$\mathrm{H}$ & -3.450577 & -1.828828 & -2.549729 \\
$\mathrm{C}$ & -5.351901 & -2.828330 & -2.231277 \\
$\mathrm{H}$ & -4.905421 & -3.826619 & -2.141868 \\
$\mathrm{H}$ & -6.165327 & -2.781014 & -1.497441 \\
$\mathrm{C}$ & -5.904433 & -2.624442 & -3.647203 \\
$\mathrm{H}$ & -6.339036 & -1.618957 & -3.728386 \\
$\mathrm{H}$ & -5.079078 & -2.658408 & -4.371392 \\
$\mathrm{H}$ & -7.338431 & -3.498784 & -5.042752 \\
$\mathrm{H}$ & -6.546044 & -4.680568 & -3.991641 \\
$\mathrm{H}$ & -7.813833 & -3.633576 & -3.343938 \\
\hline & -3.472659 & 4.240048 & -0.715105 \\
$\mathrm{H}$ & -3.831418 & 3.423082 & -2.230049 \\
$\mathrm{H}$ & -3.203074659 & 7.103177 & -3.152864 \\
$\mathrm{H}$ & 6.412038 & -2.035111 \\
$\mathrm{H}$ & 5.589378 & -3.556579
\end{tabular}

Coordinates of $(R, R)-5$ in Figure S6.

$\begin{array}{llll}\mathrm{C} & 7.632405 & -1.537244 & 2.601761 \\ \mathrm{C} & 6.233468 & -1.646816 & 2.761213 \\ \mathrm{C} & 5.376893 & -1.022718 & 1.877340 \\ \mathrm{C} & 5.865917 & -0.260658 & 0.783152\end{array}$




\begin{tabular}{|c|c|c|c|}
\hline $\mathrm{C}$ & 7.289666 & -0.097016 & 0.664398 \\
\hline $\mathrm{C}$ & 8.144015 & -0.770757 & 1.579295 \\
\hline $\mathrm{C}$ & 4.996006 & 0.400489 & -0.163287 \\
\hline $\mathrm{C}$ & 5.584868 & 1.295005 & -1.064383 \\
\hline $\mathrm{C}$ & 6.974078 & 1.464988 & -1.168534 \\
\hline $\mathrm{C}$ & 7.817013 & 0.757638 & -0.337459 \\
\hline $\mathrm{C}$ & 3.536951 & 0.213078 & -0.215320 \\
\hline $\mathrm{C}$ & 2.898242 & -1.083739 & -0.271833 \\
\hline $\mathrm{C}$ & 1.477248 & -1.143898 & -0.274685 \\
\hline $\mathrm{C}$ & 0.695544 & 0.044920 & -0.259688 \\
\hline $\mathrm{C}$ & 1.332828 & 1.298795 & -0.298689 \\
\hline $\mathrm{C}$ & 2.725406 & 1.376869 & -0.286610 \\
\hline $\mathrm{C}$ & 3.585308 & -2.318740 & -0.395639 \\
\hline $\mathrm{C}$ & 2.892262 & -3.523378 & -0.462166 \\
\hline $\mathrm{C}$ & 1.492223 & -3.577327 & -0.425262 \\
\hline $\mathrm{C}$ & 0.793696 & -2.377184 & -0.342077 \\
\hline $\mathrm{O}$ & -4.782077 & -1.972932 & -1.972070 \\
\hline $\mathrm{C}$ & -4.001628 & -3.008055 & -1.423158 \\
\hline $\mathrm{O}$ & -3.311624 & -2.621639 & -0.244956 \\
\hline $\mathrm{O}$ & -0.579044 & -2.444500 & -0.345871 \\
\hline $\mathrm{C}$ & -8.144005 & 0.770663 & 1.579289 \\
\hline $\mathrm{C}$ & -7.632386 & 1.537076 & 2.601806 \\
\hline $\mathrm{C}$ & -6.233448 & 1.646635 & 2.761257 \\
\hline $\mathrm{C}$ & -5.376880 & 1.022591 & 1.877339 \\
\hline $\mathrm{C}$ & -5.584878 & -1.294908 & -1.064561 \\
\hline $\mathrm{C}$ & -6.974090 & -1.464865 & -1.168729 \\
\hline $\mathrm{C}$ & -7.817019 & -0.757586 & -0.337586 \\
\hline $\mathrm{C}$ & -7.289664 & 0.096979 & 0.664343 \\
\hline $\mathrm{C}$ & -5.865914 & 0.260596 & 0.783112 \\
\hline $\mathrm{C}$ & -4.996006 & -0.400494 & -0.163369 \\
\hline $\mathrm{C}$ & -3.585309 & 2.318753 & -0.395253 \\
\hline $\mathrm{C}$ & -2.892264 & 3.523401 & -0.461596 \\
\hline $\mathrm{C}$ & -1.492225 & 3.577343 & -0.424707 \\
\hline $\mathrm{C}$ & -0.793696 & 2.377187 & -0.341719 \\
\hline $\mathrm{O}$ & 4.782059 & 1.973130 & -1.971809 \\
\hline $\mathrm{O}$ & 3.311622 & 2.621628 & -0.244603 \\
\hline $\mathrm{C}$ & 4.001614 & 3.008188 & -1.422765 \\
\hline
\end{tabular}




\begin{tabular}{lrrr}
$\mathrm{O}$ & 0.579044 & 2.444504 & -0.345512 \\
$\mathrm{C}$ & -1.332828 & -1.298799 & -0.298878 \\
$\mathrm{C}$ & -2.725407 & -1.376875 & -0.286810 \\
$\mathrm{C}$ & -3.536951 & -0.213091 & -0.215365 \\
$\mathrm{C}$ & -2.898240 & 1.083733 & -0.271668 \\
$\mathrm{C}$ & -1.477247 & 1.143891 & -0.274514 \\
$\mathrm{C}$ & -0.695543 & -0.044929 & -0.259696 \\
$\mathrm{H}$ & 8.299227 & -2.039039 & 3.296764 \\
$\mathrm{H}$ & 5.827472 & -2.215994 & 3.592335 \\
$\mathrm{H}$ & 4.306493 & -1.099972 & 2.027140 \\
$\mathrm{H}$ & 9.218394 & -0.653352 & 1.463294 \\
$\mathrm{H}$ & 7.355274 & 2.140402 & -1.927448 \\
$\mathrm{H}$ & 8.894178 & 0.868647 & -0.422614 \\
$\mathrm{H}$ & 4.666439 & -2.324693 & -0.445158 \\
$\mathrm{H}$ & 3.451423 & -4.449248 & -0.553252 \\
$\mathrm{H}$ & 0.950271 & -4.514499 & -0.476083 \\
$\mathrm{H}$ & -4.614349 & -3.862968 & -1.114008 \\
$\mathrm{H}$ & -3.302896 & -3.292833 & -2.217833 \\
$\mathrm{H}$ & -9.218386 & 0.653277 & 1.463280 \\
$\mathrm{H}$ & -8.299203 & 2.038829 & 3.296844 \\
$\mathrm{H}$ & -5.827445 & 2.215761 & 3.592412 \\
$\mathrm{H}$ & -4.306477 & 1.099835 & 2.027133 \\
$\mathrm{H}$ & -7.355292 & -2.140197 & -1.927713 \\
$\mathrm{H}$ & -8.894185 & -0.868573 & -0.422755 \\
$\mathrm{H}$ & -4.666443 & 2.324712 & -0.444751 \\
$\mathrm{H}$ & -3.451427 & 4.449286 & -0.552521 \\
& -0.950273 & 4.514523 & -0.475384 \\
$\mathrm{H}$ & 3.302874 & 3.293061 & -2.217400 \\
\hline .614338 & 3.863063 & -1.113518
\end{tabular}




\section{References}

(S1) Wu, T. R.; Shen, L.; Chong, M. Asymmetric allylboration of aldehydes and ketones using 3,3'-disubstitutedbinaphthol-modified boronates. Org. Lett. 2004, 6, 2701-2704.

(S2) Takaishi, K.; Takehana, R.; Ema, T. Intense excimer CPL of pyrenes linked to a quaternaphthyl. Chem. Commun. 2018, 54, 1449-1452.

(S3) Takaishi, K.; Yamamoto, T.; Hinoide, S.; Ema, T. Helical Oligonaphthodioxepins showing intense circularly polarized luminescence (CPL) in solution and in the solid state. Chem. Eur. J. 2017, 23, 9249-9252.

(S4) Zhao, D.; Wu, N.; Zhang, S.; Xi, P.; Su, X.; Lan, J.; You, J. Synthesis of phenol, aromatic ether, and benzofuran derivatives by copper-catalyzed hydroxylation of aryl halides. Angew. Chem., Int. Ed. 2009, 48, 8729-8732. 